\title{
SENSING FOREST STRUCTURE FROM LIDAR
}

Nina Amiri 



\title{
SENSING FOREST STRUCTURE FROM
} LIDAR

\section{DISSERTATION}

\author{
to obtain \\ the degree of doctor at the University of Twente, \\ on the authority of the rector magnificus, \\ prof. dr. T.T.M. Palstra, \\ on account of the decision of the Doctorate Board, \\ to be publicly defended \\ on Wednesday 8th of May 2019 at 16.45 hrs
}

by

\section{Nina Amiri}

born on January 22, 1988

in Tabriz, Iran 
This thesis is approved by:

Prof. dr. A. K. Skidmore (supervisor)

Prof. dr. Ing. P. Krzystek (co-supervisor)

ITC dissertation number 353

ITC, P.O. Box 217, 7500 AA Enschede, The Netherlands

ISBN: $\quad 978-90-365-4765-9$

DOI: $\quad$ http://dx.doi.org/10.3990/1.9789036547659

Printed by: ITC Printing Department

(C) Copyright 2019 by Nina Amiri, Enschede, The Netherlands Cover design by Benno Masselink

All rights reserved.

17 C Faculty of geo-information SCIENCE AND EARTH OBSERVATION 
Graduation committee:

\section{Chair / Secretary}

Prof. dr. A. Veldkamp

University of Twente

\section{Supervisor}

Prof. dr. A. K. Skidmore

University of Twente

Prof. dr. Ing. P. Krzystek

Munich University of Applied Sciences

\section{Members}

Prof. dr. A. Veldkamp Prof. dr. M.G. Vosselman

University of Twente University of Twente

Prof. dr. Ing. N. Pfeiffer

Vienna University of Technology

Prof. dr. L. Kooistra

Wageningen University 



\section{Acknowledgments}

I would like to express my gratitude to those who contributed to this thesis and supported my $\mathrm{PhD}$ research during these four years.

My deepest appreciate goes to my promoter, Prof. dr. Andrew Skidmore for his continuous support and motivation. Andrew, I have learned a lot about research during the discussions I had with you. I am thankful for trust you gave to me and it is been an honor for me to conduct my $\mathrm{PhD}$ under your supervision.

I would like to express my heartful gratitudes to my co-promotor Prof. dr. Peter Krzystek, the director of Laboratory of Photogrammetry and Remote Sensing at the Munich University of Applied Sciences, for giving me a chance to be his student and work as a member of his team. During my research he provided technical support and unique opportunity to work with new datasets. His friendly advices, constructive comments and reviews were extremely helpful and improved the quality of my research. Peter, what I have learned from you will lead me in my future career.

I acknowledge the Bavarian Forest National Park (BFNP) for providing precise reference data for my research. Specially, I like to thank Dr. Marco Heurich from the Bavarian Forest National Park and University of Freiburg for his guidance, assistance and expertise on forestry applications of remote sensing.

It has been a great opportunity to be a member of a research team. Therefore, I want to convey my thanks to my former colleges and friends from the Laboratory of Phtogrammetry and Remote Sensing at the Munich University of Applied Sciences. In particular to Dr. Przemslaw Polewski, for his support and valuable friendship. Without his motivation I could not bring my first scientific article from rejection to an award winning stage. Also, I would like to thank Dr. Wei Yao, Alla Serebryanyk, Sebastian Biechle, Yrneh Zarit Ulloa Torrealba and Sebastian Dersch for their friendship and collaboration. I have been lucky to come across wonderful fellows and friends at ITC as well, Dr. Elnaz Neinavaz, Dr. Xi Zhu, Dr. Roshanak Darvishzadeh, Dr. Anahita Khosravipour, Azar Zafari, Yifang Shi, Heidi Abdullah, Gara Tawanda and Jing Liu. I would like to thank them for their friendship and support. I will always remember our scientific and non-scientific discussions during Datapool meetings. I would like to appreciate the NRS Department staff, especially Esther Hondebrink and Loes Colenbrander for their enormous assistance and support in arranging all formal matters and answering my questions as an 
external $\mathrm{PhD}$ candidate.

Last but not least, I would like to express my deepest gratitude to the most valuable people in my life, who have always been there for me. My love and lifelong friend Wilhelm, no word can express how much I can appreciate your support and love. I just want to say thank you, without your support I was not be able to come to this point and complete my doctoral journey. My devoted and beloved parents in Iran, Ayoub and Nasrin and my sister Elina who have always supported me. Also, my wonderful parents in Germany, Heidrun and Norbert who never let me to forget that I can do it. This work is dedicated to my family. 


\section{Summary}

Remote sensing technology developments increase the possibility of studying the forest structure in detail and support sustainable forest management goals. The usage of remote sensing data from Laser scanning sources has also been remarkably increased for forestry applications, since conventional field inventories are time consuming and expensive. Furthermore, remote sensing-based methods for obtaining accurate and updated forest structure have been under a continuous development. The principal goal of this thesis is to develop methods using remote sensing for obtaining explicit information on forest structure such as regeneration coverage, stem count, segmented and classified tree species. The methods are applied in small test areas and can be extended to larger forest areas. The study areas are chosen from small datasets in southeast Germany, and a small forest region in Austria. A set of indicators of forest structure including regeneration coverage, stem count by segmentation and tree species are selected. A wide range of lidar data sources are employed, which could provide a high amount of relevant information for forestry applications.

The study is conducted across two temperate forest areas, and consisted of four case studies as follows. First, the regeneration coverage from airborne 3D point cloud using the enhanced 3D segmentation method (mean shift clustering combined with Normalized Cut) is estimated. A general framework is proposed for delineating detectable regeneration structures. To reduce the computational costs for the bipartition of the weighting matrix in Normalized Cuts, we combined the Normalized Cut algorithm with the mean shift clustering. The main advantage of a mean shift is to generate a small number of clusters to represent graph nodes instead of voxels. In the second study, features from high point density Airborne Laser Scanning data are used to reconstruct robust lines representing single tree stems. The components of the stem detection algorithm and the classifier parameters are learned from a training which is a three-step procedure at point level, segment level and object level. The outputs from classifier training are employed for modeling and generalizing single tree stem lines. In the 3rd phase a study is carried out to explore the potential of paraboloid surfaces for segmentation of single coniferous trees, where the static segmentation failed to partition the multiple tree clusters. The main aim is to significantly reduce over/under-segmentation. It can be expected if single trees are identified and characterized more precisely at object-based level by an evolutionary adaptive 3D segmentation. The 
applied adaptive criterion to the Normalized Cut method was thus concluded to show positive potentials towards solving over/under-segmentation issues on coniferous trees. The 4 th case study is focused on the combination of features extracted from airborne multispectral lidar and aerial imagery for detailed tree species classification. This is done through the segmentation of the $3 \mathrm{D}$ point cloud and later projection of the clusters onto the image plane to obtain bounding polygons for each tree crown. Spectral features are derived from pixels inside the bounding polygons. The process consisted of exploring a wide range of feature combinations including a feature selection step to optimize the feature space and to indicate the most relevant ones. Moreover, combining different structural and spectral features from multispectral lidar yielded more accurate results than fusing multispectral aerial imagery and single wavelength lidar data. Intensity of multispectral lidar data $(1064 \mathrm{~nm})$ was the most influential feature adding up to $10 \%$ to the classification accuracy. The experimental results showed that the lidar-based features provided the most effective information for forest structure analysis. Using the methods developed in this thesis, the approaches have the potential to be transferred to other sites. 


\section{Samenvatting}

Ontwikkelingen op het gebied van teledetectie-technologie vergroten de mogelijkheid om de bosstructuur in detail te kunnen bestuderen en duurzame bosbeheerdoelen te kunnen ondersteunen. De belangstelling voor het gebruik van teledetectiegegevens van laserscanningsbronnen is ook opmerkelijk toegenomen voor toepassingen in de bosomgeving. Momenteel zijn de gebruikelijke methoden afhankelijk van hoge kosten van conventionele veldinventarissen. Bovendien zijn op afstandsensoren gebaseerde methoden voor het bestuderen van een nauwkeurige en bijgewerkte bosstructuur continu in ontwikkeling geweest. Het belangrijkste doel van dit proefschrift is om methoden te ontwikkelen met behulp van teledetectie voor het verkrijgen van expliciete informatie over de bosstructuur, zoals regeneratiedekking, stamtelling, gesegmenteerde en geclassificeerde boomsoorten. De methoden worden toegepast in kleine testgebieden en kunnen worden uitgebreid naar grotere bosgebieden. De studiegebieden zijn gekozen uit kleine datasets in Zuidoost-Duitsland en een kleine bosregio in Oostenrijk. Een reeks indicatoren van bosstructuur inclusief regeneratiebedekking, stamtelling per segmentatie en boomsoorten worden geselecteerd. Er wordt een breed scala aan lidargegevensbronnen gebruikt, die een grote hoeveelheid relevante informatie zouden kunnen opleveren voor bosbouwtoepassingen.

De studie werd uitgevoerd in twee gematigde bosgebieden en bestond uit vier deel-onderzoeken. In het eerste onderzoek wordt de regeneratiedekking van $3 \mathrm{D}$-puntwolken in de lucht met behulp van de verbeterde 3D- segmentatiemethode (gemiddelde verschuivingsclustering gecombineerd met normalised Cut) geschat. Een algemeen kader wordt voorgesteld voor het afbakenen van detecteerbare regeneratiestructuren. Om de computationele kosten voor de tweedeling van de wegingsmatrix in genormaliseerde bezuinigingen te verminderen, hebben we het genormaliseerde-snij-algoritme gecombineerd met de gemiddelde verschuivingsclustering. Het grootste voordeel van een gemiddelde verschuiving is om een klein aantal clusters te genereren om grafische knooppunten te representeren in plaats van voxels. In het tweede onderzoek worden functies van Laser Scanning-gegevens met hoge puntdichtheid in de lucht gebruikt voor het reconstrueren van robuuste lijnen die enkelvoudige boomstelen voorstellen. De componenten van het stamdetectiealgoritme en de classificatorparameters worden geleerd van een training die een drietrapsprocedure is op puntniveau, segmentniveau en objectniveau. De outputs van de classificatietraining worden gebruikt voor het modelleren en 
generaliseren van enkelvoudige stamlijnen. Het derde onderzoek werd gedaan naar het potentieel van paraboloïde oppervlakken voor de segmentatie van enkele naaldbomen, waarbij de statische segmentatie de meerdere boomclusters niet kon partitioneren. Het belangrijkste doel is om over/onder-segmentatie aanzienlijk te verminderen. Het kan worden verwacht als afzonderlijke bomen op object-gebaseerd niveau nauwkeuriger worden gedentificeerd en gekenmerkt door een evolutionair adaptieve 3D-segmentatie. Het toegepaste adaptieve criterium voor de methode Normalized Cut werd zo geconcludeerd dat het positieve mogelijkheden toonde voor het oplossen van problemen bij over/onder-verdeling van naaldbomen. Het vierde onderzoek concentreert zich op de combinatie van kenmerken die zijn verkregen uit multispectrale lidar en luchtfoto's van de lucht voor gedetailleerde classificatie van boomsoorten. Dit wordt gedaan door de segmentatie van de 3D-puntwolk en later de projectie van de clusters op het afbeeldingsvlak om begrenzende polygonen voor elke boomkroon te verkrijgen. Spectrale functies zijn afgeleid van pixels binnen de begrenzende polygonen. Het proces bestond uit het verkennen van een breed scala aan functiecombinaties, waaronder een functiekeuzestap om de speelruimte te optimaliseren en de meest relevante aan te geven. Bovendien leverde het combineren van verschillende structurele en spectrale kenmerken van multispectrale lidar meer accurate resultaten dan het samensmelten van multispectrale luchtfoto's en lidar-data met één golflengte. Intensiteit van multispectrale lidar-gegevens $(1064 \mathrm{~nm})$ was de meest invloedrijke functie tot $10 \%$ aan de nauwkeurigheid van de classificatie. De experimentele resultaten toonden aan dat de lidar-gebaseerde kenmerken de meest effectieve informatie voor analyse van de bosstructuur opleverden. Met behulp van de methoden die in dit proefschrift zijn ontwikkeld, kunnen de benaderingen worden overgedragen naar andere gebieden. 


\section{Zusammenfassung}

Aktuelle Entwicklungen in der Fernerkundung ermöglichen neuerdings, Waldstrukturen im Detail zu analysieren und damit eine Basis für ein nachhaltiges Forstmanagement zu schaffen. Fernerkundungsdaten von flugzeugetragenen Laserscannern für Anwendungen im Bereich der Waldökologie zu verwenden ist von zunehmenden Interesse, da konventionelle Bestandsaufnahmen zeitaufwendig und teuer sind. Der Einsatz der Fernerkundung spielt dabei eine entscheidende Rolle, um Methoden zur Lösung der technischen Probleme zu entwickeln. Darüber hinaus wurden Fernerkundungsverfahren zur präzisen und aktuellen Gewinnung Waldstrukturen kontinuierlich weiterentwickelt. Das Hauptziel dieser Arbeit ist die Entwicklung von Fernerkundungsmethoden zur Erlangung expliziter Informationen über die Waldstruktur wie Regeneration, Stammanzahl, segmentierte und klassifizierte Baumarten. Die Methoden werden in kleinen Testgebieten angewendet und können auf größere Waldflächen ausgedehnt werden. Die verwendeten Datensätzen beziehen sich auf ein Untersuchungsgebiet im Südosten Deutschlands (Nationalpark Bayerischer wald) und ein kleines Testgebiet in Österreich. Indikatoren für Waldstrukturen, darunter Verjüngung, Stammanzahl, segmentierte Einzelbäume und klassifizierte Baumarten. Umfangreiche Fernerkundungsdatensätze konnten genutzt werden, die eine große Menge relevanter Informationen für Forstanwendungen darstellten.

Die Studie wurde in zwei Testgebieten durchgeführt und untersucht die folgenden 4 Szenarien. Als erstes wurde die nachwachsende Verjüngung mithilfe einer modifizierten 3D Segmentierungsmethode aus einer 3D Punktwolke bestimmt. Ein allgemeine Strategie wird vorgeschlagen, um detektierbare Verjüngungsstrukturen zu erkennen. Um die Rechenzeit des Normalized Cuts Verfahrens zu reduzieren, werden der Normalized Cut Algorithmus mit dem Mean Shift Clustering verfahren kombiniert. Die Verwendung der Mean Shift Cluster besitzt den Vorteil, dass eine kleinere Anzahl von Supervoxeln für die Erstellung der Gewichtsmatrix verwendet werden kann. In der zweiten Fallstudie wurden Merkmale aus hochaufösenden Airborne Laser Scanning Daten berechnet, um einzelne Baumstämme aus der 3D Punkwolke zu rekonstruieren. Trainingsdaten werden verwendet, um Klassifikatoren des Stammerkennungsalgorithmus zu trainieren der als 3-Stüfiges Verfahren, einen punkt-basierten, segment-basierten und objekt-basierten Teil beinhaltet. Die Resultate des Klassifizierertrainings werden genutzt um einzelne Stammlinien zu modellieren und zu generalisieren. In der dritten Studie 
werden parabolischen Oberflächen verwendet, um die Segmentierung von einzelnen Nadelbäumen zu verbessern, die wegen des statischen Abbruchkriteriums zu unbefriedigenden Ergebnissen führen kann. Das Hauptziel ist es, die Über- und Untersegmentierung signifikant zu reduzieren. Dies kann erwartet werden, falls einzelne Bäume präzise auf der Objektebene mittels einer adaptiven 3D Segmentierung charakterisiert werden können. Die Ergebnisse zeigen, dass das adaptive Abbruchkriterium bei der Normalized Cut Methode zu einer Reduktion der Über- und Untersegmentierung bei Nadelbäumen führt. Die vierte Fallstudie verwendete multispektrale Laserscanningdaten und Luftbilder für eine detaillierte Baumartenklassifikation. Hierbei wurden die Umringpolygone der Baumcluster, die über eine Segmentierung der 3D Punktwolke berechnet werden, auf die Bildebene projiziert. Spektrale Merkmale wurden aus den Pixeln innerhalb der Begrenzungspolygone abgeleitet. Der gesamte Prozess beinhaltete die Untersuchung einer umfangreichen Merkmalsraums mithilfe einer vorwärtsgerichteten Merkmalsselektion, die den Merkmalsraum optimiert und die relevantesten Merkmale selektiert. Die Untersuchungen zeigen, dass die Kombination von verschiedenen strukturellen und spektralen Merkmalen, die aus multispektralem lidar berechnet werden, genauere Ergebnisse erbringt als die Fusion von multispektralen Luftbildern und lidar Daten einer einzelnen Wellenlänge. Die Intensität von multispektralem lidar (1064nm) erweist sich als das einflussreichste Merkmal, was zu einer 10\%igen Genauigkeitssteigerung führt. Die experimentellen Ergebnisse zeigen, dass die lidar-basierten Merkmale die wichtigsten Informationsträger zur Waldstrukturanalyse sind. Die in dieser Arbeit entwickelten Methoden besitzen das Potential, auf andere Gebiete übertragen zu werden. 


\section{Contents}

Summary iii

Samenvatting v v v v v

Zusammenfassung vii

Contents

$\begin{array}{lr}\text { List of Figures } & 6\end{array}$

$\begin{array}{lr}\text { List of Tables } & 7\end{array}$

List of Abbreviations $\quad 9$

1 Introduction 11

1.1 Importance of forest structure . . . . . . . . . . . . . . . 12

1.2 Lidar remote sensing of forest structure . . . . . . . . . . . . . 13

1.3 Research objectives . . . . . . . . . . . . . . . 16

1.4 Study area . . . . . . . . . . . . . . . . . 16

1.5 Thesis outline . . . . . . . . . . . . . . . 18

2 Estimation of regeneration coverage in a temperate forest by $3 \mathrm{D}$ segmentation $\quad 19$

2.1 Introduction . . . . . . . . . . . . . . . . . . . 21

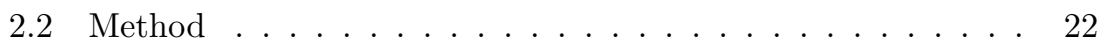

2.3 Materials . . . . . . . . . . . . . . . . . . . . 29

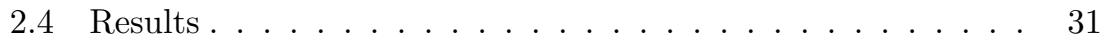

2.5 Discussion . . . . . . . . . . . . . . . . . . . . . 36

2.6 Conclusions . . . . . . . . . . . . . . . . . . 38

3 Detection of single tree stems in forested areas from high $\begin{array}{ll}\text { density ALS point clouds } & 41\end{array}$

3.1 Introduction . . . . . . . . . . . . . . . . . 43

3.2 Method .......................... 45

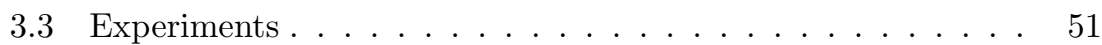

3.4 Results and Discussion . . . . . . . . . . . . . . . . . 53

3.5 Conclusions ..................... 56 
4 Adaptive stopping criterion for top-down segmentation of ALS point clouds

4.1 Introduction . . . . . . . . . . . . . . . . 59

4.2 Top-down segmentation . . . . . . . . . . . . . 61

4.3 Adaptive stopping criterion . . . . . . . . . . . . . 62

4.4 Experiment . . . . . . . . . . . . . . . . 69

4.5 Results and discussion . . . . . . . . . . . . . . . . 72

4.6 Conclusions . . . . . . . . . . . . . . . 79

5 Tree species classification by fusing multispectral lidar and aerial imagery

5.1 Introduction . . . . . . . . . . . . . . . . 83

5.2 Related work ................... 83

5.3 Experiment . . . . . . . . . . . . . . . 86

5.4 Method ........................ 89

5.5 Results and discussion ... . . . . . . . . . . 100

5.6 Conclusions . . . . . . . . . . . . . . . . 107

5.7 Acknowledgment . . . . . . . . . . . . . . 108

6 Synthesis 109

6.1 Introduction . . . . . . . . . . . . . . 110

6.2 Estimation of regeneration coverage in a temperate forest by 3D segmentation . . . . . . . . . . . . . 110

6.3 Detection of single tree stems in forested areas from high density ALS point clouds . . . . . . . . . . . . . . . 113

6.4 Adaptive stopping criterion for top-down segmentation of ALS point clouds . . . . . . . . . . . . . . . . . . 115

6.5 Tree species classification by fusing multispectral lidar and aerial imagery . . . . . . . . . . . . . . . . 118

6.6 Implications of lidar for forest structure analysis in ecological studies ...................... 119

6.7 Further research .................. 122

$\begin{array}{lr}\text { Bibliography } & 123\end{array}$

$\begin{array}{llr}7 & \text { Biography } & 141\end{array}$ 


\section{List of Figures}

1.1 Location of the Bavarian Forest National Park, Germany. . . . . 17

1.2 Location of the Hochficht forest, Austria. . . . . . . . . . . . . . 18

2.1 (a) Cylinder-shaped kernel with mean shift vector in 3D space; (b) 2D Gaussian kernel profile, where the horizontal axis represents the distance $d$ from the kernel center $X$ to a point $X_{i} ; d=\left\|X-X_{i}\right\| .25$

2.2 Horizontal and vertical quadratic distances between mean shift clusters in 3D space; Credits: Yao et al. (2013). . . . . . . . . . . 26

2.3 Dependence of prior knowledge term $M(i, j)$ of clusters $i$ and $j$ on their distance to the local maximum of CHM. . . . . . . . . . 27

2.4 Height filtering of $5 \mathrm{~m}$ on the tree segments in a sample plot with $20 \%$ reference regeneration coverage; (a) overview of the sample plot (b) visualization of the 3D point clouds before and (c) after applying the height filter in the sample plot. . . . . . . . . . 28

2.5 Estimation of regeneration coverage by $3 \mathrm{D}$ segmentation in the 2012 dataset vs. reference data. . . . . . . . . . . . . 31

2.6 Estimation of regeneration coverage by $3 \mathrm{D}$ segmentation (a) in the 2011 and (b) 2009 datasets vs. reference data. . . . . . . . . 32

2.7 Effect of overstory density on the accuracy of estimated regeneration coverage. . . . . . . . . . . . . . . . 33

2.8 Effect of deciduous tree species composition in the overstory on the accuracy of estimated regeneration coverage. . . . . . . . . 33

2.9 Sensitivity analysis of the normalized cut threshold $N C u t_{\text {Thres }}$ and the minimum number of points in a segment $N c u t_{\text {min }}$ points parameters. . . . . . . . . . . . . . 34

2.10 Sensitivity analysis for the 2012 dataset, (a) with respect to the mean shift kernel radius $h_{r}$ and $(H=4 \mathrm{~m})$; (b) with respect to the mean shift kernel height $H$ and $\left(h_{r}=2.4 \mathrm{~m}\right) . \ldots . . . . .35$

2.11 Sensitivity analysis of the mean shift kernel parameters as Completeness, Correctness and $F_{1}$ score (a) for the 2009 and (b) 2011 datasets. . . . . . . . . . . . . . . 37

3.1 Overview of stem detection pipeline. . . . . . . . . . . . . . . 46

3.2 Cylindrical 3D Shape Context around a segment. . . . . . . . . . 47 
3.3 The aggregate distance $d$ between two segments; the angular deviation $d_{A}$ is shown with green arrow and the spatial distance between point centroids $d_{C}$ with red arrow. . . . . . . . . . .

3.4 The stopping criterion for clustering the segments. The green lines represent the segments in cluster $C_{i}$ and the magenta lines show the segments in cluster $C_{j}$, respectively. The red line is the line fitted to both clusters' points. The red points outside the cylinder have projected distance greater than $d_{p, \max } \ldots \ldots$. . . . . . .

3.5 A point cloud visualization of sample forest scene with multiple visible stems (point clouds of scene colored by height over DTM).

3.6 The detection results for a sample plot:(a), (b) and (c) correspond to point, segment and object levels, respectively (see Sections 3.2.1-3.2.3). At point level in (a), the red color shows low and blue high probability. The solid green bars in (b) indicate tree stems classified as positive and red bars refer to unmatched tree stems with references. The points which do not belong to the detected stems are removed from analysis and colored as cyan. Orange ellipses outline examples of the false alarms. The fitted magenta lines in (c) represent the reference tree stems which overlap with colored detected stems (ODR with the $\ell_{1}$ norm). . . . . . . .

3.7 Single tree stem detection performance with probability threshold of 0.4 to 0.6 for the test plots. . . . . . . . . . . . . . .

4.1 Overview of single tree segmentation strategy using adaptive stopping criterion. . . . . . . . . . . . . . .

4.2 Detected local maxima for two pair of clusters $S_{i} ; S_{j}$. The $S_{i}$ represents a cluster with 2 true tree tops; the $S_{j}$ shows a false positive scenario in the cluster with at least one false detected local maximum. . . . . . . . . . . . . . . . . . . . .

4.3 Fitted paraboloid surfaces on detected local maxima. The $S_{i}$ represents a cluster with two true tree tops; the $S_{j}$ shows a false positive cluster with at least one wrong detected local maximum.

4.4 Signed distances between data points (in red) and their approximate projections (in yellow) onto the fitted paraboloid (green). Positive and negative distances are indicated respectively by blue and red lines. (a) Residuals around a true tree top, distributed symmetrically around zero, (b) Residuals around false local maximum located at the side of the tree, showing bias towards large positive values. . . . . . . . . . . . . . . .

4.5 Estimating the spatial overlap ratio $o p_{r}$ between fitted paraboloid surfaces. The $S_{i}$ represents a cluster with 2 true tree tops; the $S_{j}$ shows a false positive cluster with at least one false detected local maximum. . . . . . . . . . . . . . . . 
4.6 The entire volume of a paraboloid $V$ can be decomposed into an infinite number of elliptical slices with infinitely small thickness $d z$ and volumes $v(z)$ which are functions of their heights $z$. $H$ represents the height of the paraboloid. The number of points generated in each slice should be proportional to its cross section area. . . . . . . . . . . . . . . . .

4.7 ALS point clouds of Plot A (100\% coniferous), Plot B (99\% coniferous) and Plot $\mathrm{C}(70 \%$ coniferous $)$ colored by height over DTM.

4.8 ROC curve of single tree adaptive segmentation for the plots A ( $100 \%$ coniferous), B (99\% coniferous) and C (70\% coniferous). Each diagram contains 4 ROC curves which correspond to various thresholds of the maximum overlap ratio $\max _{o p_{r}}$. . . . .

4.9 ROC curve of single tree adaptive segmentation for the plots A $(100 \%$ coniferous $), \mathrm{B}(99 \%$ coniferous $)$ and C (70\% coniferous). Each diagram contains 4 ROC curves which correspond to various thresholds of the minimum probability threshold $\min _{p_{t h r}}$.

4.10 Single tree segmentation results for a part of plot A: (a) corresponds to the normalized cut segmentation with mean shift clustering results; and (b) represents the adaptive stopping criterion for normalized cut segmentation by fitting paraboloids, respectively. Each set of colored points represents a delineated single tree. The red boxes outline the over-segmentation issue on an example single tree. . . . . . . . . . . . . .

4.11 Single tree segmentation results for a part of plot A: (a) corresponds to the normalized cut segmentation with mean shift clustering results; and (b) represents the adaptive stopping criterion for the normalized cut segmentation by fitting paraboloids, respectively. Each set of colored points shows a delineated single tree. The red boxes outline the under-segmentation issue on an example group of single trees. . . . . . . . . . . . . . .

5.1 Color infrared orthophotograph of the Bavarian Forest National Park (park area in green), with the test area containing sample plots for classifying tree species marked in yellow. . . . . . . . . .

5.2 A forest scene in the color infrared aerial image with a ground pixel resolution of $20 \mathrm{~cm}$. Snags (standing dead spruce trees with crowns) appear in gray, while the living trees are shown in red (broad-leaf and coniferous trees). . . . . . . . . . . .

5.3 Vertical profile of a forest scene in the 3D point cloud; a) $532 \mathrm{~nm}$ spectral wavelength, b) addition of the $1064 \mathrm{~nm}$ wavelength, and c) the $1550 \mathrm{~nm}$ wavelength is combined with the 532 and 1064 nm wavelengths. . . . . . . . . . . . . .

5.4 The four selected small target areas located on the airfield that were used for the amplitude calibration $\left(a_{r e f}\right) . \ldots . . . . .992$

5.5 Overview of the feature-based strategy for tree species classification. 92 
5.6 Learning curves describing the average error as a function of feature count; $P C L-M S L S$ refers to the MSLS point cloud, Img is the aerial imagery, $P C L-C h 2$ refers to single wavelength channel $C h 2$, and $B o W$ refers to the BoW model with various neighborhood sizes. The curves correspond to the classification performance in terms of average error (\%) after feature selection step using features extracted from a) the MSLS point cloud and b) the aerial imagery and single wavelength channel $C h 2 . \quad \ldots .105$

5.7 Classified individual tree classes for a sample area in Bavarian Forest National Park (spectral wavelength of $1064 \mathrm{~nm}$ ). . . . . 107

6.1 An example forest scene with regeneration in the Bavarian Forest National Park. . . . . . . . . . . . . . . . . . . . . . 111

6.2 3D point cloud (30 point $\left./ \mathrm{m}^{2}\right)$ visualization of two sample circular plots with a) absence and b) presence of the regeneration (point clouds are colored by height over DTM). . . . . . . . . . . . . . 112

6.3 3D visualization of single trees in the high density ALS data with visible stems (point cloud is colored by height over DTM). . . . . 114

6.4 a) Visualization of single tree segmentation on a sample area dominated by broad-leaf trees. Correctness and completeness results on the sample plot based on the b) local maxima and c) stem positions combined with local maxima (Dersch, 2018). . . . 115

6.5 Example depiction of geometry shapes of coniferous and broad-leaf trees. . . . . . . . . . . . . . . . . 116

6.6 An example coniferous tree cluster in a point cloud where the adaptive segmentation and paraboloid fitting was not fully successful in decision level to continue partitioning (the red cylinder shows the detected local maximum for the cluster). . . . . . . . . 117 


\section{List of Tables}

2.1 Different ALS campaigns. . . . . . . . . . . . . . . . 30

3.1 Control parameters for the single tree stem detection method; $n_{p}$ refers to the number of points inside the clusters. . . . . . . . . 53

4.1 Properties of sample plots. . . . . . . . . . . . . . . . . 70

4.2 Control parameters of the adaptive stopping criterion method for single tree segmentation. . . . . . . . . . . . . . . . 72

4.3 Results of analysis on the upper canopy layer for the sample plots A, B and C. . . . . . . . . . . . . . . . . 73

5.1 Flight campaigns of multispectral lidar data. . . . . . . . . . . 87

5.2 The number of matched trees in the upper canopy layer with the overall tree species percentage in the plots. . . . . . . . . . . 89

5.3 Main control parameters for the single tree segmentation using the Normalized Cut method. . . . . . . . . . . . . . . . . . . 93

5.4 Description of the generated features for each tree segment $i$. . . 94

5.5 Results of tree species classification. Best results are highlighted in bold. . . . . . . . . . . . . . . . . . . . . . 99

5.6 Confusion matrix of classification performance from scenario 4 using features extracted from multispectral imagery. . . . . . . . 101

5.7 Confusion matrix of classification performance from scenario 8 using features extracted from MSLS point cloud. . . . . . . . . . 102

5.8 Confusion matrix of classification performance from scenario 10 using combined features extracted from MSLS point cloud and multispectral imagery. . . . . . . . . . . . . . . . . . 102

5.9 List of the most important features under different classification scenarios (each scenario is defined in Table 5.5). Each scenario has 30 features selected via the feature selection step. The below mentioned feature abbreviations are explained in subsec.5.4.4. . . 103 



\section{List of Abbreviations}

$\begin{array}{ll}\text { ALS } & \text { Airborne Laser Scanning } \\ \text { CHM } & \text { Canopy Height Model } \\ \text { CSC } & \text { Cylindrical 3d Shape Context } \\ \text { DBH } & \text { Diameter at Breast Height } \\ \text { DEMs } & \text { Digital Elevation Models } \\ \text { DTM } & \text { Digital Terrain Model } \\ \text { GPS } & \text { Geographical Positioning System } \\ \text { INS } & \text { Inertial Navigation System } \\ \text { KLR } & \text { Kernelized Logistic Regression } \\ \text { MSLS } & \text { Multispectral Laser Scanning } \\ \text { NIR } & \text { Near Infrared } \\ \text { ODR } & \text { Orthogonal Distance Regression } \\ \text { PFH } & \text { Point Feature Histograms } \\ \text { RANSAC } & \text { RANdom SAmple Consensus } \\ \text { RGB } & \text { Red Green Blue } \\ \text { RMSD } & \text { Root Mean Square Deviation } \\ \text { SWIR } & \text { Short Wave Infrared } \\ \text { TLS } & \text { Terrestrial Laser Scanning }\end{array}$





\section{Introduction}




\subsection{Importance of forest structure}

Forests across the globe have an important role in several activities such as ecosystem management, biomass production and biodiversity monitoring. The forest coverage is subject to permanent change at different temporal and spatial scales (Coppin and Bauer, 1996). Since the present-day forest inventory requires accurate and continuously updated information it is necessary to frequently measure essential forest variables (Asner and Martin, 2016).

In this context, the term forest structure refers to a general topic in forestry and ecology community. Different studies have provided comprehensive summarizes on the available definitions and attributes. The forest structure is not a measurable quantity by itself. However, properties of it can be described by means of a wide variety of variables such as species distribution, vertical and horizontal spatial patterns, tree attributes, stand volume and/or combinations of them at different canopy levels (McElhinny et al., 2005). Regardless of the definitions, updated information on the forest structure variables is needed to improve forest management strategies (Koch et al., 2009) by quantifying the current condition for predicting future stand dynamics.

A forest is often described by a set of general characteristics including composition, function and structure (Franklin et al., 1981). Beside composition (which is described as presence/dominance of species or by relative indices of biodiversity) and function (explains types and rates of a process such as biomass), the physical forest characteristics are explained under the general concept of forest structure. The structural elements at tree level are described by various attributes associated with spatio-temporal properties, such as height, canopy closure and species composition (Latifi et al., 2015a). Qualification of these elements can provide valuable information in order to assess future forest planning and management goals.

Forest structure analysis is mainly focused on gathering detailed, precise and updated information on vertical and horizontal structure of forest layers (Latifi et al., 2017). Among the different structural variables, four are investigated in this thesis to describe the vertical and horizontal structure of canopy at different layers, namely 1) regeneration coverage, 2) tree stem count per ha, 3) tree segmentation and 4) tree species classification. The first variable refers to the proportion of the regeneration coverage which is essential for the forest biodiversity while the following ones express the structural attributes at single tree level. Conventional approaches for estimation of the forest structure variables are costly and time consuming and they are limited to small spatial extents. Remote sensing techniques based on laser scanning as well as optical imagery can help to overcome the above mentioned limitations, allowing for large-scale mapping of forest structure variables and reduce the cost of measurements. 


\subsection{Lidar remote sensing of forest structure}

Recently, remote sensing technology has become practical for application in forestry due to the ability of repetitive coverage at short time intervals while providing accurate characteristics at different scales (Mas, 1999; Latifi, 2012; White et al., 2016). This provides objective and practical solutions for developing and maintaining forest structure analysis, which in turn supports a variety of management purposes. Different types of remote sensing data including optical, thermal, Synthetic Aperture Radar (SAR), and Airborne Laser Scanning (ALS) from medium to fine spatial resolution are required to directly estimate the structural variables. Among remote sensing techniques, ALS, also known as airborne lidar (Light Detection And Ranging), has rapidly gained popularity over the last two decades in the forestry community due to its unique capability to provide a quantitative 3D digital representation of the objects of interest (Hyyppä et al., 2008; Vosselman and Maas, 2010; White et al., 2016; Krzystek and Polewski, 2017).

ALS is an active remote sensing technique that is not affected by solar illumination or shadowing, and was introduced in the 1980s for the purpose of large-scale monitoring (Woodhouse et al., 2011). The large amount of detail obtainable with lidar as well as its applicability in hard to access forested areas resulted in new demands that cannot be achieved by traditional methods. Furthermore, lidar can provide both horizontal and vertical information in 3D space, enabling the elimination of the influence of background, understory, and canopy geometry (Morsdorf et al., 2006). The ability to penetrate partially the vegetation and to capture 3D structural and spatial information has brought lidar to the stage of a powerful tool for forest structure characterization (Lefsky et al., 1999). Depending on the application, lidar sensors can be used with Unmanned Aerial Vehicles (UAVs) and Terrestrial Laser Scanning (TLS) or mounted on airborne (ALS) and space-born platforms. Initially, lidar was used for mapping terrain surfaces, especially in forestry applications. Forest structure analysis using lidar data has been investigated for management purposes at both plot and single tree level to identify the important structural variables (Heurich, 2008; Korpela et al., 2010b; Yao et al., 2012). A forest canopy, due to its complexity, tends to produce a denser distribution of returns (Hardiman et al., 2013). Recent developments in lidar technology have generated new full waveform scanners that can record more efficiently the backscatter pulses within the travel path of a laser beam to provide additional information about the characteristics of reflected objects and a higher spatial point density (Reitberger et al., 2009; Yao and Stilla, 2010; Wallace et al., 2012; Gong et al., 2015; Scaioni et al., 2018). Furthermore, in certain practical applications Single-Photon lidar (532 $\mathrm{nm}$ green laser) and Geiger-mode lidar (1064 nm IR laser) have recently gained popularity (Swatantran et al., 2016; Stoker et al., 2016; Harding et al., 2011). Both Geiger and Single-Photon lidar systems utilize focal plane array detectors and record the returned laser pulse using an array of receivers instead of a single receiver. A receiver array can detect individual photons and count photons per detector. These sensors are operated at higher flying 
altitudes and use lower laser power compared to linear mode lidar systems (Polewski, 2017; Stoker et al., 2016). Although these new sensors have shown some promising results in preliminary studies, the data acquired during day time is still too noisy for commercial uses (Stoker et al., 2016). Geiger-mode systems in particular, are impractical for analysis of vegetation coverage. Note that throughout the work reported in this thesis, Geiger and Single-Photon lidar systems were not used in any of the experiments.

\subsubsection{Lidar remote sensing for regeneration coverage:}

The distribution and coverage analysis of the understory canopy layer can be estimated by means of lidar remote sensing. However, methods for regeneration coverage prediction across temperate forests have not been fully explored (Hill et al., 2017). Due to recent developments in full waveform lidar, the laser beam now has a high degree of probability to travel through the canopy, reaching young trees (regeneration) and providing a stronger ground return signal (Lefsky et al., 2002a; Pirotti, 2011; Amiri et al., 2016).

The focus of lidar-based studies on extracting information about forest understory coverage has been on either separating the over- and understory layers from each other (Zimble et al., 2003; Mund et al., 2015) or on estimating the probability of the areas in which the understory can exhale (Hill and Broughton, 2009). Regarding the understory, there is great practical interest in forest ecology due to the fact that the variations along the vertical structure of the understory is a relevant criterion later for the occurrence of dominant tree species in the overstory canopy layer (Falkowski et al., 2009). Besides the vertical structure, accurate information on the horizontal structure of the understory canopy underneath the top tree layer, has a significant role across temperate mixed stands, not only for the woody species, but for all the herbal species close to the forest ground as well (Latifi et al., 2017).

\subsubsection{Lidar remote sensing for stem detection:}

The reconstruction of single tree stems at different canopy layers is an important factor for providing essential habitat resources for further studies (McElhinny et al., 2005; Polewski et al., 2015b; Polewski, 2017). Standard ALS sensors with a point density up to 30 points $/ \mathrm{m}^{2}$ are able to cover large areas more efficiently in terms of time and expense. However, the ALS-based methods fail to detect tree stems due to the low point density at the level of the intermediate-/understory canopy layer (Vierling et al., 2013). The high point density lidar can fill the gap between costly traditional field measurements and low-resolution lidar data by providing a variety of intermediate-/understory canopy layer attributes at a fine scale. In the case of high point density ALS data, the laser beam has a better chance to penetrate the vegetation canopy and to provide ground information, depending on parameters such as flight altitude, pulse repetition frequency, and laser power. This enables the detection of single tree stems more precisely compared to the common ALS data (point density around 30 points $/ \mathrm{m}^{2}$ ). Due to the 3D nature of the point cloud and smaller laser footprint, from the method point of view it is 
possible to capture the information of single tree stems at intermediate and understory canopy layers.

\subsubsection{Lidar remote sensing for tree segmentation and classification:}

Over time, a large number of methods have been developed for segmentation at single tree level. The advantages and disadvantages of a particular method can greatly affect the results of the single tree delineation and later species classification. In the same environment, different approaches may yield different results. Therefore, it is important to select an appropriate segmentation method depending on circumstances such as technical capabilities, phenology of forests, or cost of data. There have been a variety of methods developed for single tree segmentation applied directly on 3D point clouds or on the derived Canopy Height Model (CHM). The variables obtained from the segmentation method using lidar data can be later used for additional approaches such as understory coverage estimation and tree species classification (Zhao et al., 2009; Yao et al., 2012; Amiri et al., 2016). From the accuracy point of view, the segmentation is in an early stage and requires further research.

In practice, top-down segmentation methods such as Normalized Cut (NCut) (Reitberger et al., 2009) applied directly on 3D point clouds for single tree delineation tend to produce over- and under-segmentation errors based on the forest characteristic and empirically defined control parameters. Even after extensive calibration of the most relevant parameters using reference data, broad-leaf trees with large crowns might be split into several tree segments or coniferous trees might be merged into a single tree segment. These errors later lead to incorrect estimation of timber volume. Amiri et al. (2018b) has recently shown that the Normalized Cut segmentation used mainly for coniferous trees can be enhanced by using an adaptive criterion approach instead of using a static Normalized Cut threshold.

Previous studies have shown that vegetation species diversity in temperate forests can be substantially affected by changes in the forest structure (Ellison et al., 2005). Different structural features related to tree crowns and height can be derived from a 3D ALS point cloud and can be afterwards considered for tree species classification. The initial idea for using lidar features for classification was related to the different crown and height properties of various tree species (Ørka et al., 2010). Beyond the multi-echo detection capability, lidar may also provide valuable information on overlapped crowns of different tree species. The 3D coordinates, intensity, and pulse width features are obtained using a Gaussian waveform decomposition into a sum of components (Mallet and Bretar, 2009). The decomposition is used to characterize the different target objects along the path of a laser beam (Guo et al., 2011). The common full waveform lidar features for classification purposes are 3D coordinates, amplitudes, echo widths, cross-sections, and echo shapes (Mallet et al., 2008). The amplitude and echo width are commonly used for single tree species identification (Korpela et al., 2010b). In addition, the intensity recorded by lidar sensors is a function of the reflectance property of an object (Lefsky et al., 1999). The intensity is to some extent insensitive to ambient light and atmospheric conditions (Killinger and Meyuk, 1987; Kasparian 
et al., 2003). This reliability enables intensity to be used for spectral separation between objects. Recent studies have shown that the lidar intensity is the key element for distinguishing between tree species, particularly in conjunction with structural variables (Kim et al., 2009; Yu et al., 2017; Shi et al., 2018a). Moreover, a feature selection step that aims to select the most relevant features has made a significant contribution to the final classification results (Amiri et al., 2018a). Note that the accuracy of the single tree species classification relies mainly on the single tree detection and segmentation (Hyyppä et al., 2008). However, as mentioned before, tree segmentation can result in large errors in terms of over/under-segmentation in complex and dense forests where tree crowns are overlapped (Zhao et al., 2009).

Furthermore, the use of the multi-sensor (ALS and optical imagery) datasets for forest applications has been a matter of interest due to the possibility of combining structural and spectral information. However, in the fusion approaches, some factors related to the integration of geometry and spectral characteristics of the datasets may still affect the process of extracting accurate tree boundaries for the classification step by producing errors. Although the single wavelength lidar data is promising (up to $90 \%$ overall accuracy) for classification of broad-leaf and coniferous trees, the conducted analysis proved that detailed spectral information is necessary for multiple tree species classification (Yao et al., 2012). Therefore, the addition of the extracted spectral features from available multispectral lidar data for tree species classification in temperate forests is highly recommended.

\subsection{Research objectives}

In this thesis, the primary aims were to:

- estimate the regeneration coverage of the understory canopy layer using an enhanced 3D segmentation method and ALS point clouds.

- detect single tree stems using a feature-based classifier strategy at point, segment, and object levels by using high density ALS point clouds.

- enhance the over/under-segmentation problems mainly for the coniferous tree species using an adaptive stopping criterion approach for top-down segmentation of ALS point clouds.

- investigate the contribution of the features extracted from multispectral lidar data and aerial imagery, to the classification accuracy of multiple tree species.

\subsection{Study area}

Considering the research objectives and the availability of data, Chapters 2, 4 , and 5 are focused on the Bavarian Forest National Park (BFNP). The main test site of this thesis was located in southeast Germany along its border with the Czech Republic $\left(49^{\circ} 3^{\prime} 19^{\prime \prime}\right.$ N, $13^{\circ} 12^{\prime} 9^{\prime \prime}$ E) (Fig.1.1). This mixed mountain forest is geographically characterized as a part of the Bohemian 
Forest region. Elevation in the BFNP ranges from 600 to $1453 \mathrm{~m}$ above sea level. The climate of the region is temperate, and precipitation varies from 1200 to $1800 \mathrm{~mm} /$ year. The minimum average annual temperature is between $3^{\circ} \mathrm{C}$ and $6^{\circ} \mathrm{C}$. In general, the predominant tree species in the BFNP are Norway spruce (67\%), European beech (24.5\%), and Silver fir $(2.6 \%)$ (Heurich et al., 2010).

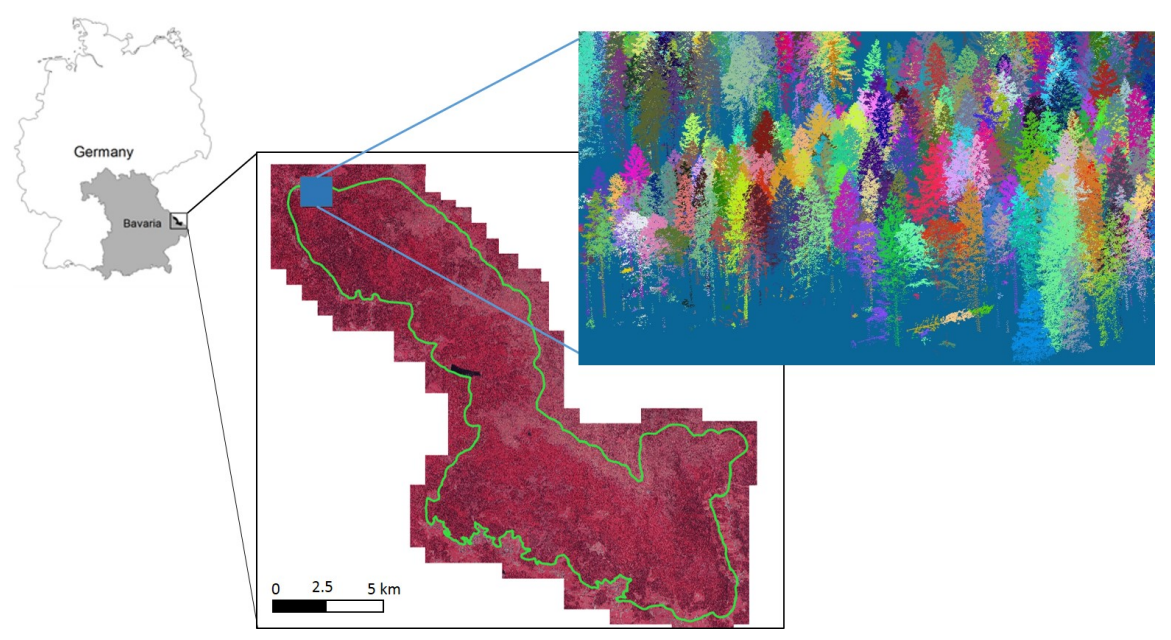

Figure 1.1: Location of the Bavarian Forest National Park, Germany.

As another test site away from the Bohemian Forest region, the lidar data in Chapter 3 were acquired from the Hochficht forest. The area is located along the border of Austria and the Czech Republic (48 $44^{\prime} 11^{\prime \prime} \mathrm{N}, 13^{\circ} 55^{\prime} 16^{\prime \prime}$ E) (Fig.1.2). The highest recorded elevation of the forest is $1,338 \mathrm{~m}$ above sea level. The temperature is especially changing with altitude: the average temperature is approximately $6^{\circ} \mathrm{C}$ at an altitude of $750 \mathrm{~m}$ and about $3^{\circ} \mathrm{C}$ at an altitude of $1,300 \mathrm{~m}$. The forest is a mixture of broad-leaf and coniferous tree species. 


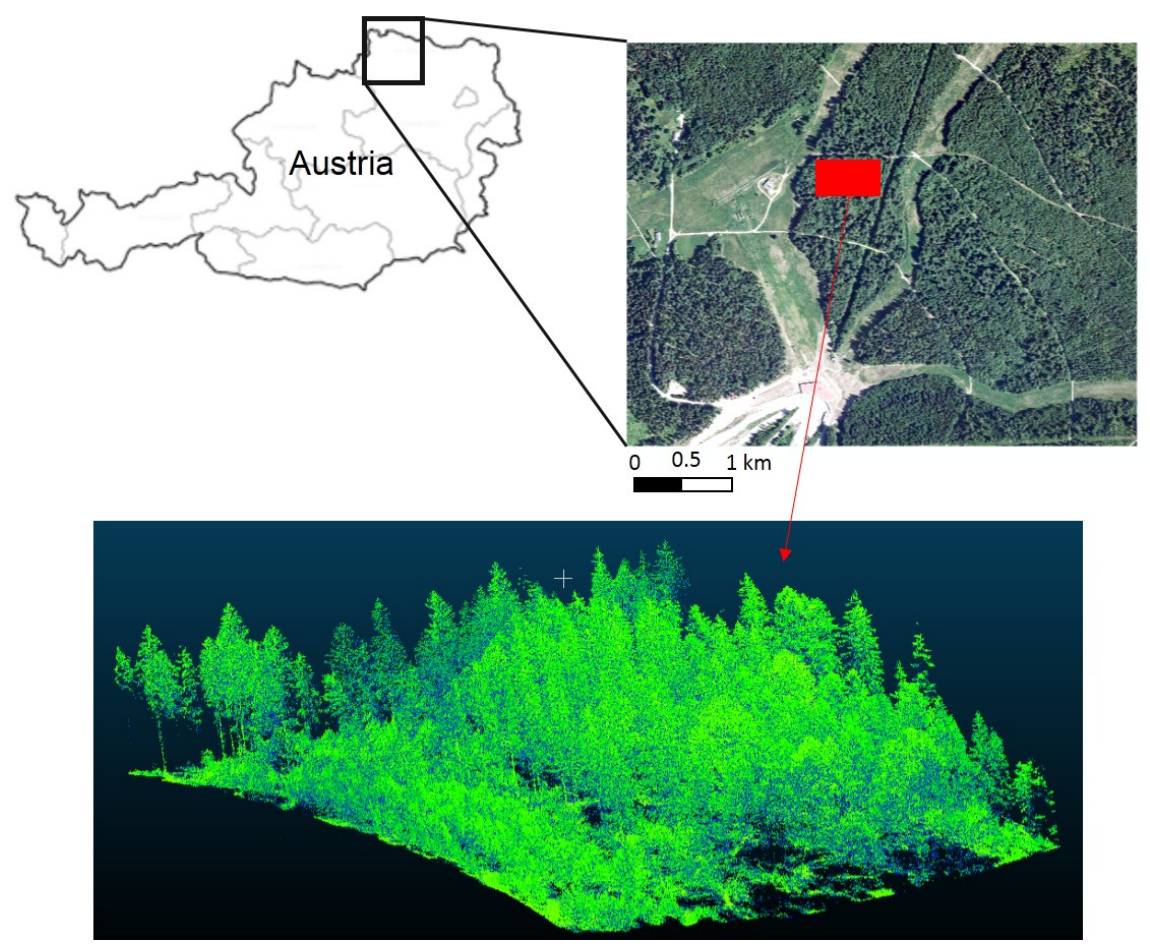

Figure 1.2: Location of the Hochficht forest, Austria.

\subsection{Thesis outline}

This thesis comprises six chapters, including a general introduction, four core chapters, and a synthesis. Each core chapter has been provided as a standalone research article that has been published or submitted to peer-reviewed ISI journals. The structure of the chapters is as follows. Chapter 1 presents the research background, main objectives, and the thesis outline. Chapter 2 presents the enhanced 3D segmentation approach using lidar data for estimation of regeneration coverage in the understory canopy layer under leafon conditions. Chapter 3 introduces a three step classifier training approach using 3D shape descriptors to extract single tree stems in high point density ALS data. Chapter 4 evaluates the novel adaptive stopping criterion for a top-down segmentation approach focused on coniferous trees and examines the utility of quadratic surfaces for classifier training. Chapter 5 describes multiple tree species classification using a feature-based strategy extracted from multispectral lidar data and aerial imagery. Finally, Chapter 6 provides an overview of the significant research findings of the thesis. The contributions of the studies mentioned in this thesis for the forest structure analysis and management are discussed. Furthermore, future research possibilities that could lead to further enhancement of the methods are described. 


\section{Estimation of regeneration coverage in a temperate forest by 3D segmentation}

This chapter is based on:

Amiri, N., Yao, W., Heurich, M. \& Krzystek, P. (2015). Regeneration detection by 3D segmentation in a temperate forest using airborne full waveform Lidar data. Proceedings of SilviLaser 2015: 14th conference on lidar applications for assessing and managing forest ecosystems: 28-30 September 2015, La Grande Motte, France.

Amiri, N., Yao, W., Heurich, M., Krzystek, P., \& Skidmore, A. K. (2016). Estimation of regeneration coverage in a temperate forest by $3 \mathrm{D}$ segmentation using airborne laser scanning data. International journal of applied earth observation and geoinformation, 52, 252-262. DOI: $10.1016 /$ j.jag.2016.06.022 


\begin{abstract}
Forest understory and regeneration are important factors in sustainable forest management. However, understanding their spatial distribution in multilayered forests requires accurate and continuously updated field data, which are difficult and time-consuming to obtain. Therefore, cost-efficient inventory methods are required, and Airborne Laser Scanning (ALS) is a promising tool for obtaining such information. In this study, we examine a clustering-based 3D segmentation in combination with ALS data for regeneration coverage estimation in a multilayered temperate forest. The core of our method is a two-tiered segmentation of the $3 \mathrm{D}$ point clouds into segments associated with regeneration trees. First, small parts of trees (super-voxels) are constructed through mean shift clustering, a nonparametric procedure for finding the local maxima of a density function. In the second step, we form a graph based on the mean shift clusters and merge them into larger segments using the normalized cut algorithm. These segments are used to obtain regeneration coverage of the target plot. Results show that, based on validation data from field inventory and Terrestrial Laser Scanning (TLS), our approach correctly estimates up to $70 \%$ of regeneration coverage across the plots with different properties, such as tree height and tree species. The proposed method is negatively impacted by the density of the overstory because of decreasing ground point density. In addition, the estimated coverage has a strong relationship with the overstory tree species composition.
\end{abstract}




\subsection{Introduction}

The process of forest regeneration, understanding as young trees below $5 \mathrm{~m}$ height, is a central component in forest succession, which ensures a continuous forest cover and adds structural complexity (Swanson et al., 2010). Therefore, it plays an important role in maintaining biological diversity in forest ecosystems. Regeneration appears either as advanced regeneration under canopy gaps or on open areas after large scale natural disturbance and forest management actions. Information on understory vegetation can benefit the assessment of tree species richness and vertical structure of forests (Wing et al., 2012).

Conventional forest inventory of the understory is based on limited sample plots, which are used to calculate means and confidence intervals for the larger forest areas. However, one of the main disadvantages of this approach was the limited number of plots which often covered less than a few percent of the total forest area (Köhl et al., 2006). Therefore, it was not possible to extract area-wide information. In addition, ground-based methods for understory inventory are generally time-consuming and labor-intensive (Tuanmu et al., 2010; Wing et al., 2012). Remote sensing can provide objective, cost-effective, and practical solutions for developing and maintaining automated area-wide forest mapping (Janssen and Huurneman, 2000). Passive remote sensing methods are useful alternative tools for gathering information across large areas (Wing et al., 2012). However, because of the complexity of the overstory, they cannot penetrate the forest ground layer.

ALS systems with direct measurement of 3D structural information have been shown to be a promising tool for characterizing vegetation (Lefsky et al., $2002 \mathrm{~b})$. The decomposition of waveforms can overcome the limitations of conventional first/last pulse data in the analysis of the forest understory by providing the intensity and pulse width (Reitberger et al., 2009). In addition, based on former approaches, the waveform decomposition process gives higher spatial point density and information about the vertical structure of the understory (Yao et al., 2013). Therefore, after waveform decomposition, full waveform ALS data are more representative in multilayered forests (Eskelson et al., 2011; Latifi et al., 2015b).

Efforts towards the application of ALS data for understory and regeneration studies have been promoted in earlier research. However, so far, only limited experiments have been done to estimate regeneration coverage in multilayered forests using ALS data (Yao et al., 2013). Su and Bork (2007) examined the CHM (Canopy Height Model) frequency histograms of a first/last pulse ALS system and tested two thresholds of $0.3 \mathrm{~m}$ and $1.3 \mathrm{~m}$ to separate the overstory and understory in aspen forests. Hill and Broughton (2009) detected the presence of regeneration in temperate broadleaf forests, using leaf-off and leaf-on ALS data. Later, Morsdorf et al. (2010) tested both height and intensity information to detect canopy layers (understory and a shrub layer) in a dry Mediterranean forest. They applied a supervised cluster analysis, assuming that intensity measurements of some tree species can improve the classification; however, they achieved an accuracy rate of $48 \%$ in detecting understory. Korpela et al. (2012) used ALS data to 
study the understory by designing a conceptual model for the transmission losses of laser pulse intensity through the overstory. Their results showed that it is impossible to obtain normalized second-return intensity data from the forest floor or ground vegetation. Ferraz et al. (2012) used mean shift clustering on ALS data in a multilayered forest to detect suppressed trees, and achieved a detection rate of $12.8 \%$. Yao et al. (2013) noted the potential of height distributions and geometric properties for regeneration detection using full waveform ALS data. Latifi et al. (2015b) highlighted the value of Lidar metrics for characterizing the structural properties of the lower forest layer in temperate mixed stands. These studies indicate that the problem of correctly detecting regeneration using ALS height distributions is related to the presence of ground vegetation and overlapping crowns in the overstory. Although the availability of ALS data and appropriate post-processing methods has increased, there is still limited experience in applying them to estimate regeneration coverage in multilayered forests. In the mean shift clustering-based approach on ALS data, multiple clusters may correspond to a single tree. This fragmentation in the understory level makes it difficult to distinguish whether the cluster belongs to the regeneration or it is a part of neighboring single tree. Therefore, an approach that can provide regeneration coverage based on automated segmentation of forest understory structures in 3D space is needed.

The objectives of this study are (i) to estimate the regeneration coverage with an adapted 3D segmentation algorithm using full waveform ALS data, (ii) to investigate the effect of overstory density and tree species composition on the accuracy of estimated regeneration coverage. Moreover, we provide a sensitivity analysis for specific control parameters of the method.

The remainder of this work is structured as follows: Section 2 describes the details of our approach; Section 3 illustrates the study area, materials, and field measurements. The results are presented in Section 4 and discussed in Section 5. Finally, the conclusions are stated in Section 6.

\subsection{Method}

We use an adapted 3D segmentation algorithm (see (Yao et al., 2013)), to estimate the regeneration coverage (the ratio between the area covered by regeneration trees and the total area of the sample plot). The $3 \mathrm{D}$ segmentation algorithm is a two-tiered segmentation procedure. The normalized cut segmentation as the core part of our method is computationally expensive. To reduce the computational costs for the bipartition of the weighting matrix in normalized cuts, we combine the normalized cut segmentation with the mean shift clustering. The advantage of a mean shift is to generate a small number of clusters to represent the graph nodes instead of voxels. The former approach by (Yao et al., 2013) also confirmed that the mean shift clusters can better indicate smaller trees in the understory, which is not possible to do using the normalized cut segmentation based solely on voxels. The ground truth data is available as proportional values of regeneration coverage. The steps of the entire procedure are as follows: (i) above-ground height threshold 
determination, (ii) local tree maxima filtering, (iii) mean shift clustering, (iv) feature derivation for mean shift clusters, (v) normalized cut segmentation , and (vi) height filtering of the segmentation results. In the following, we explain the steps of the adapted 3D segmentation.

\subsubsection{Above-ground height threshold}

The input data after waveform decomposition with superimposed Gaussian functions is a set of $3 \mathrm{D}$ point clouds with $3 \mathrm{D}$ coordinates $X_{i}\left(x_{i}, y_{i}, z_{i}\right)$ and two physical properties (intensity and pulse width) for each point (Reitberger et al., 2009). The derived 3D point clouds from decomposition contain the entire overstory, understory, and ground vegetation (herbal layer). To focus on regeneration, we need to remove the points belonging to the ground vegetation up to a height of $1 \mathrm{~m}$ from the ground surface level, which is estimated from a given Digital Terrain Model (DTM). This height threshold value in the $3 \mathrm{D}$ segmentation affects the results of the regeneration coverage estimation by commission and omission errors.

\subsubsection{Local tree maxima filtering}

In our approach, within each grid cell, the highest 3D point is estimated from a given DTM. The local maxima positions are provided by the watershed segmentation on the Canopy Height Model (CHM), and act as prior knowledge to detect where the overstory trees are located (see (Reitberger et al., 2009)). This prior knowledge is directly included in the similarity function of normalized cut segmentation (see Section 2.4).

\subsubsection{Mean shift clustering}

In forestry applications, ALS point clouds are noted as a multimodal distribution where each mode as a local maximum both in height and density, corresponding to a crown top (Ferraz et al., 2012). Our experiment investigates the ability of mean shift clustering combined with the normalized cut segmentation to extract the modes of point clouds in each cluster. Although the mean shift procedure is able to define the modes of a density function, this procedure requires a testing framework because of the complexity of the understory. Here, our approach is to segment vertical and horizontal structures of the forest understory in $3 \mathrm{D}$ space.

The mean shift is a nonparametric, feature-space clustering technique, which neither requires prior knowledge of the cluster number nor constrains the shape of the clusters (Comaniciu and Meer, 2002; Wu and Yang, 2007). For each point in $3 \mathrm{D}$ space, the mean shift in a defined kernel window performs a gradient ascent procedure on the local estimated density until convergence to the local maxima. In this technique, for given $n$ points of $X_{i}, i=1,2, \ldots, n$ in $3 \mathrm{D}$ space, the probability density function with the obtained kernel $K(X)$ is:

$$
f_{h_{r}, K}(X)=\frac{1}{n h_{r}^{3}} \sum_{i=1}^{n} K\left(\frac{X-X_{i}}{h_{r}}\right)
$$


where $h_{r}$ as the kernel bandwidth is the smoothing parameter that specifies the contribution of each point in Eq. 2.1. The kernel bandwidth $h_{r}$ refers to the kernel radius. $K$ is a nonlinear function of the distance from the points in 3D space to the center of kernel $X$. The modes of the density function are located at the zeros of the gradient function $\nabla f(X)=0$. Assuming that $g$ is the derivative of the kernel profile $k, g(X)=-k^{\prime}(X)$ (see (Yao et al., 2013); Comaniciu and Meer (2002)), the mean shift vector is calculated as:

$$
m_{h_{r}}(X)=\frac{\sum_{i=1}^{n} X_{i} g\left(\left\|\left(\frac{X-X_{i}}{h_{r}}\right)\right\|^{2}\right)}{\sum_{i=1}^{n} g\left(\left\|\left(\frac{X-X_{i}}{h_{r}}\right)\right\|^{2}\right)}-X
$$

In Eq. 2.2 the most computationally expensive component of the mean shift vector is related to the identification of neighboring points in $3 \mathrm{D}$ space, which is defined as the kernel radius $h_{r}$ (Yao et al., 2013). This parameter depends on the local forest structure (Ferraz et al., 2012). Therefore, we use a cylinder-shaped kernel because it can model the shape of a tree better than a spherical kernel. The kernel radius $h_{r}$ affects the segmentation procedure and has to be set in advance by empirical experiments. The determination of the optimal value is based on the assumed reasonable size of the regeneration trees which we want to cluster (Yao et al., 2013).

Besides the radius $h_{r}$, the height of kernel $H$ is also an important parameter, which has to be defined in advance. The kernel height $H$ is included in the Gaussian function of the mean shift vector to determine whether or not a point $X_{i}$ belongs to the cylinder-shaped kernel. In our study, the Gaussian kernel profile $g$ is defined as:

$$
\begin{cases}g\left(\frac{\left\|X-X_{i}\right\|^{2}}{h_{r}}\right)=e^{\frac{-\left\|X-X_{i}\right\|}{2 h_{r}^{2}}} & \text { for } \quad X_{i} \in \text { Cylinder }\left\{h_{r}, H\right\} \\ g\left(\frac{\left\|X-X_{i}\right\|^{2}}{h_{r}}\right)=0 & \text { for } \quad X_{i} \notin \text { Cylinder }\left\{h_{r}, H\right\}\end{cases}
$$

For any point in the 3D space located within the cylinder-shaped kernel with the predefined kernel height $H$, the Gaussian kernel profile in Eq. 2.3 is applied. Fig. 3.1a shows the cylinder-shaped kernel for the density estimation with the mean shift vector, and Fig. 3.1b presents the 2D Gaussian kernel profile. The mean shift vector always points toward the direction of the maximum increase in density (see (Ferraz et al., 2012)).

\subsubsection{Feature derivation for mean shift clusters and normalized cut segmentation}

After applying the mean shift to the 3D point clouds, for each cluster, we estimate four groups of features. These features are: (i) $S^{h p}=\left\{S^{x}, S^{y}\right\}$ to describe the horizontal position of the clusters, (ii) $S^{v p}=\left\{S^{z}\right\}$ to describe their vertical position, (iii) $S^{I}=\left\{S^{I}\right\}$ as an overall mean intensity for the entire mean shift cluster, and $S^{W}=\left\{S^{W}\right\}$ as a mean pulse width for the entire cluster.

The normalized cut segmentation (see (Shi and Malik, 2000)) is a spectral 
a)

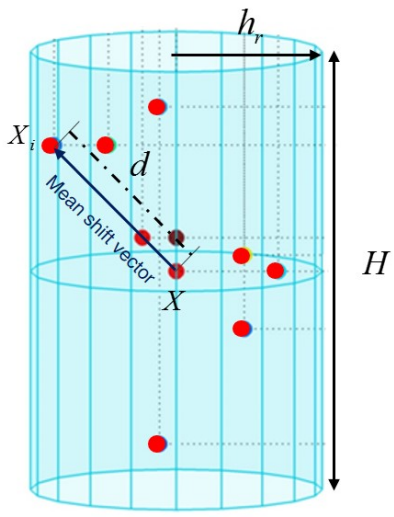

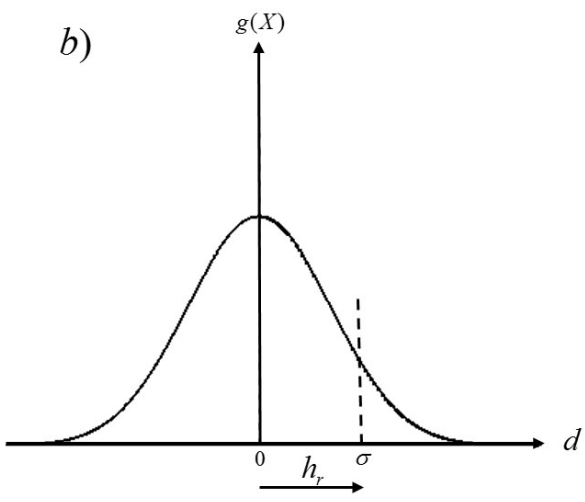

Figure 2.1: (a) Cylinder-shaped kernel with mean shift vector in 3D space; (b) 2D Gaussian kernel profile, where the horizontal axis represents the distance $d$ from the kernel center $X$ to a point $X_{i} ; d=\left\|X-X_{i}\right\|$.

method for data clustering. The two disjointed segments $A$ and $B$ of the graph are found by maximizing the similarity of the connected clusters and minimizing the similarity between segments $A$ and $B$ (see (Reitberger et al., 2009)). The corresponding normalized cut function is:

$$
\operatorname{NCut}(A, B)=\frac{C u t(A, B)}{\operatorname{Assoc}(A, V)}+\frac{C u t(A, B)}{\operatorname{Assoc}(B, V)}
$$

with $C u t(A, B)=\sum_{i \in A, j \in B} w_{i j}$ as the total sum of the weights between the segment $A$ and $B$, while $A \operatorname{ssoc}(A, V)=\sum_{i \in A, j \in V} w_{i j}$ is the sum of weights of all edges ending in segment $A$ in Eq. 2.4 (see (Reitberger et al., 2009; Yao et al., 2013)). This property uses the eigenstructure of the object similarity function to construct a low-dimensional representation of the points (Polewski et al., 2015a). The normalized cut segmentation is applied to the mean shift clusters. The similarity function for normalized cut is based on the pairwise similarity of the clusters. The mentioned segmentation is controlled by several parameters whose values can be optimized in experiments. The most important control parameter that controls the subdivision of the graph is the normalized cut threshold $\mathrm{NCut}_{\text {Thres }}$. Another one is the minimum number of points in a segment $N C u t_{\text {minpoints. }}$. In this study, these parameters are empirically defined.

In order to set up the weighting matrix $W$, we introduce the function

$$
w(i, j)= \begin{cases}e^{-P(i, j)} \times e^{-Z(i, j)} \times e^{-F(i, j)} \times e^{-M(i, j)} & \text { if } D_{i j}^{x y}<r_{x y} \\ 0 & \text { otherwise }\end{cases}
$$


with

$$
\begin{gathered}
P(i, j)=\left(\frac{D_{i j}^{h p}}{\sigma_{h p}}\right)^{2}, Z(i, j)=\left(\frac{D_{i j}^{v p}}{\sigma_{v p}}\right)^{2}, \\
F(i, j)=\left(\frac{D_{i j}^{I W}}{\sigma_{I W}}\right)^{2}, M(i, j)=\left(\frac{D_{i j}^{\max }}{\sigma_{M}}\right)^{2}
\end{gathered}
$$

that consists of similarities $w_{i j}$ between mean shift clusters $i$ and $j$, which are located within a cylindrical volume of predefined radius and unlimited height around cluster $i$. The $D_{i j}^{x y}$ in Eq. 2.5 describes the horizontal distance between clusters $i$ and $j$. The $P(i, j)$ and $Z(i, j)$ elements weight the quadratic Euclidean distances between the clusters. The component $D_{i j}^{h p}=\left\|S_{i}^{h p}-S_{j}^{h p}\right\|$ is the horizontal, and $D_{i j}^{v p}=\left\|S_{i}^{v p}-S_{j}^{v p}\right\|$ the vertical distance between clusters $i$ and $j$. They are weighted separately in consideration that trees generally have greater height than width. An increase in the vertical distance between two clusters $i$ and $j$ has to lead to an attenuation of their weights $w_{i j}$, but with a smaller magnitude compared to the horizontal distance. Here, the knowledge on the appearance of trees is used implicitly. Fig. 3.2 presents the horizontal and vertical quadratic distances between clusters to further construct the normalized cut segments.
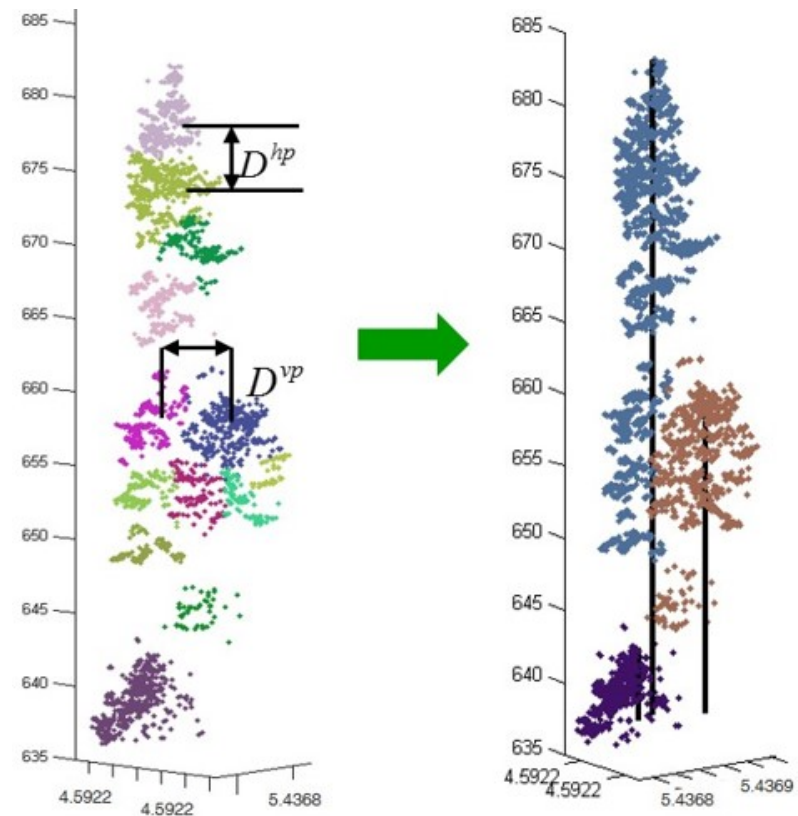

Figure 2.2: Horizontal and vertical quadratic distances between mean shift clusters in 3D space; Credits: Yao et al. (2013).

The $F(i, j)$ element is the quadratic Euclidean distance between two arbitrary feature vectors derived from the $3 \mathrm{D}$ point clouds. In Eq. 2.7, the $D_{i j}^{I W}$ describes the quadratic distance between the mean intensity and mean pulse width of 
clusters $i$ and $j$, as follows:

$$
F(i, j)=\frac{\left(S_{i}^{I}-S_{j}^{I}\right)^{2}+\left(S_{i}^{W}-S_{j}^{W}\right)^{2}}{\sigma_{I W}^{2}}
$$

Furthermore, the $M(i, j)$ element is the largest horizontal distance of two mean shift clusters $i$ and $j$ to the closest local maximum of CHM. By including this element into the similarity function, we are encoding prior knowledge about likely tree positions obtained from watershed segmentation, as the local maxima in CHM correspond, with high probability, to dominant overstory trees (see (Reitberger et al., 2009)). Fig. 3.3 illustrates the $M(i, j)$ element in the weighting matrix $W$. The parameters $\sigma_{h p}, \sigma_{v p}, \sigma_{I W}$, and $\sigma_{M}$ control the sensitivity of the impact factors $P(i, j), Z(i, j), F(i, j)$, and $M(i, j)$ in Eq. 2.6.

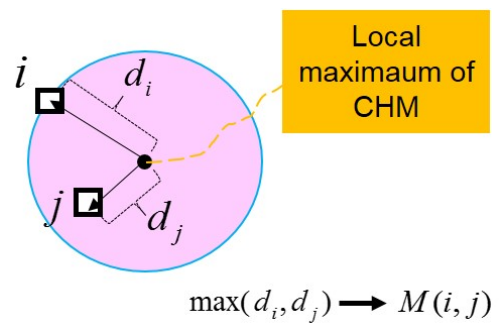

Figure 2.3: Dependence of prior knowledge term $M(i, j)$ of clusters $i$ and $j$ on their distance to the local maximum of CHM.

\subsubsection{Height filtering of tree segments}

We filter the segments generated in the previous step by removing those with heights greater than $5 \mathrm{~m}$. The remaining segments contain laser points corresponding to the regeneration trees. Fig. 3.4 shows the $3 \mathrm{D}$ point clouds in a sample plot associated with the segments before and after height filtering of the segmentation results.

The $5 \mathrm{~m}$ height filtering excludes points that belong to the dominating trees in the overstory. It is not sufficient to apply a simple height filter to the ALS point cloud height distributions, as this is unlikely to distinguish whether a return came from the lower parts of the overstory or from the adjacent surface of the understory (Hill and Broughton, 2009). In addition, a significant number of segments that are extracted with direct height filtering as regeneration are likely to overlap or cover the same subsets of point clouds belonging to the single trees. Therefore, the disadvantage of direct height filtering of the $3 \mathrm{D}$ point clouds would be a high commission error rate in the regeneration coverage results.

In each sample plot, the remaining 3D segments that are very likely to be regeneration are converted to $2 \mathrm{D}$ polygons, and their area is calculated. The regeneration coverage is the ratio between the areas covered by the remaining 
segments to the total area of same plot. Later, for comparison with the reference data, the results are presented in percentages.

\subsubsection{Evaluation}

The evaluation is performed based on the area covered by regeneration in percentage, which is compared to the reference data for each dataset separately. In the 2012 dataset, the reference data for the regeneration at single-tree level (geographical coordinates) is not available. Therefore, the root mean square deviation (RMSD) method is applied to represent the differences between the estimated regeneration coverage by the $3 \mathrm{D}$ segmentation and the reference data. Eq. 2.8 shows the RMSD:

$$
R M S D=\sqrt{\frac{\sum_{q=1}^{n^{\prime}}\left(\hat{E}_{q}-E_{q}\right)^{2}}{n^{\prime}}}
$$

where $n^{\prime}$ is the number of sample plots, $E_{q}$ is the reference regeneration coverage for the $q^{\text {th }}$ sample plot, and $\hat{E}_{q}$ is the estimated regeneration coverage by the $3 \mathrm{D}$ segmentation.
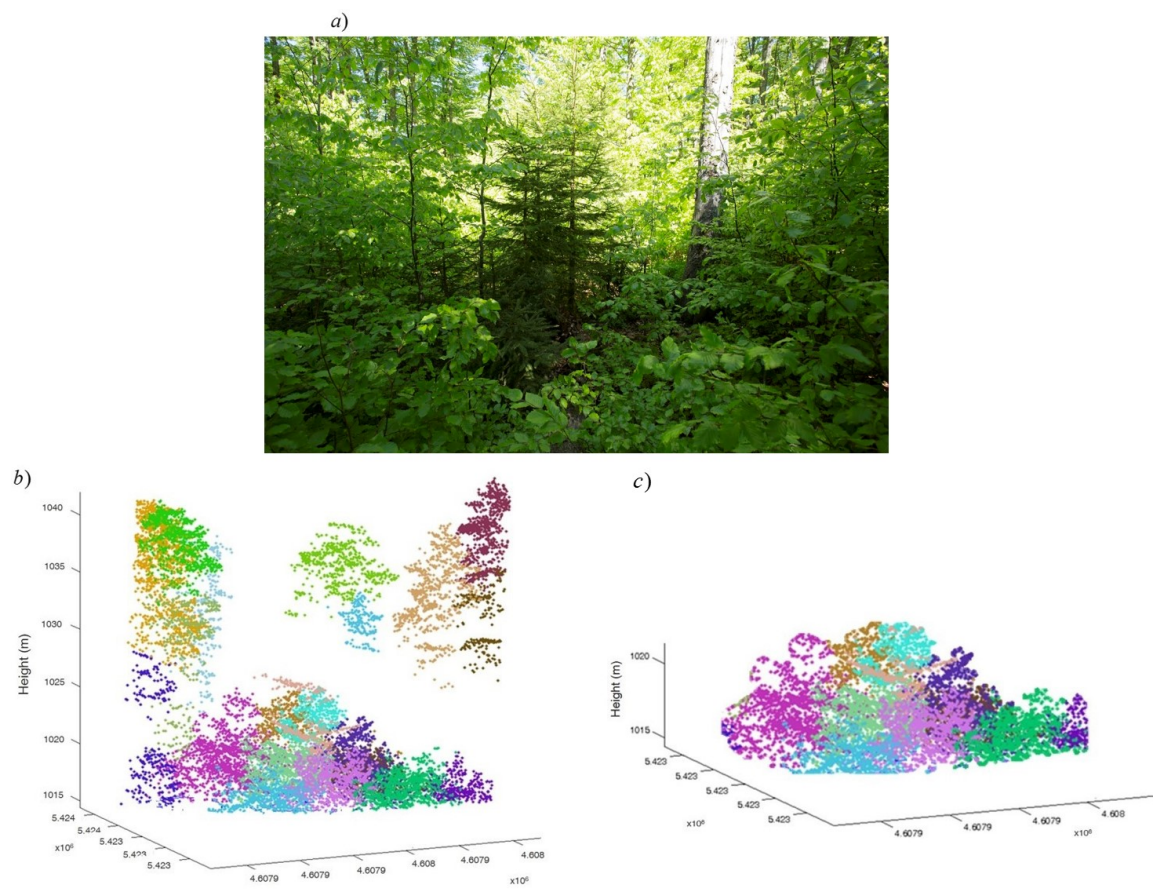

Figure 2.4: Height filtering of $5 \mathrm{~m}$ on the tree segments in a sample plot with $20 \%$ reference regeneration coverage; (a) overview of the sample plot (b) visualization of the $3 \mathrm{D}$ point clouds before and (c) after applying the height filter in the sample plot.

For the 2011 and 2009 datasets, because of the availability of the reference data at the stand level, we use the "completeness" and "correctness" measures to 
characterize the performance of the 3D segmentation. The "completeness" and "correctness" are defined in Eq. 2.9 and 2.10, respectively. The regeneration segments (areas) estimated by the 3D segmentation that overlap by more than $50 \%$ with the reference regeneration segments are called Area TP (TP term refers to the true positives). In Eq. 2.9, the Area $a_{\text {reference regeneration segments }}$ component describes the total area of the reference regeneration segments,

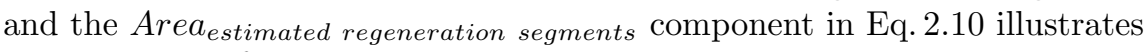
the total area of the segments estimated by the 3D segmentation.

$$
\begin{aligned}
& \text { Completeness }=\frac{\text { Area }_{T P}}{\text { Area }_{\text {reference regeneration segments }}} \\
& \text { Correctness }=\frac{\text { Area }_{T P}}{\text { Area }_{\text {estimated regeneration segments }}}
\end{aligned}
$$

The accuracy of the estimated regeneration coverage is defined as the estimated regeneration coverage from the $3 \mathrm{D}$ segmentation with the optimal values for the control parameters divided by the reference data in the same plot. Furthermore, the $F_{1}$ score value for the 2009 and 2011 dataset is calculated to estimate the optimal kernel radius $h_{r}$ and height $H$. Eq. 2.11 shows the $F_{1}$ score:

$$
F_{1} \text { score }=\frac{2 T P}{2 T P+F N+F P}
$$

where $T P$ is the True Positives, $F N$ is the False Negatives and the $F P$ is the False Positives of the estimated results by the 3D segmentation.

\subsection{Materials}

\subsubsection{Study area}

Our experiments were conducted in the Bavarian Forest National Park $\left(49^{\circ} 3^{\prime} 19^{\prime \prime} \mathrm{N}, 13^{\circ} 12^{\prime} 9^{\prime \prime} \mathrm{E}\right)$, a temperate forest located in the southeastern part of Germany along the border with the Czech Republic, during three time periods (2009, 2011, and 2012). The elevation of the Bavarian Forest National Park varies between $600 \mathrm{~m}$ and $1453 \mathrm{~m}$. The study area contains a mixture of mountainous and subalpine forest types with a high structural complexity, dominated by Norway spruce (Picea abies) and European beech (Fagus sylvatica) (Heurich et al., 2010).

\subsubsection{Full waveform ALS data}

ALS data were acquired by the Milan Flug GmbH Company with the Riegl LMS-680i and LMS-560Q (full waveform) scanners. Table 2.1 contains the details of the acquisition flights, flying heights, and point densities. These datasets did not allow multi-temporal ALS data analysis, since the reference 
data for the same sample plots were not available. For the 2009 and 2011 datasets, only a single plot was present respectively. This had to be considered when comparing the results of the regeneration coverage of other datasets to these. The collected waveforms were decomposed with superimposed

Table 2.1: Different ALS campaigns.

\begin{tabular}{cccc}
\hline \hline Flight time & 2009 & 2011 & 2012 \\
\hline Riegl Scanner type & LMS-560Q & LMS-560Q & LMS-680i \\
Foliage condition & Leaf-on & Leaf-off & Leaf-on \\
Flight height & $400 \mathrm{~m}$ & $400 \mathrm{~m}$ & $600 \mathrm{~m}$ \\
Pts $/ \mathrm{m}^{2}$ & 25 & 25 & 30 \\
Reference data & TLS $^{*}$ data & TLS* data & Field inventory \\
\hline * Terrestrial Laser Scanning; & & &
\end{tabular}

Gaussian functions (see (Reitberger et al., 2009)), resulting in the 3D point clouds (see (Polewski et al., 2015a)). Furthermore, a DTM with a grid size of $1 \mathrm{~m}$ and absolute accuracy of $25 \mathrm{~cm}$ was generated by in-house filtering algorithms of the data provider for all the sample plots (see (Reitberger et al., 2009; Heurich, 2006)). This was generated from the acquired ALS data in 2003. The calibration for the intensity and pulse width was achieved using the intensity and pulse width of the emitted Gaussian pulse with the help of a calibration flight at the airport (see (Yao et al., 2013)).

\subsubsection{Reference data}

The reference data of the study for the 2012 dataset were recorded by proportional values using the ocular method on sample plots in 2013. The method was based on a visual estimation of the area occupied by the canopy cover of each forest layer (see (Paletto and Tosi, 2009)) using the principle of the Braun-Blanquet method (Braun-Blanquet, 1928). In each plot, between 1 to $40 \%$ coniferous and 1 to $50 \%$ deciduous regeneration coverages were recorded. For the 2011 and 2009 datasets, the reference data were captured by multi-station Terrestrial Laser Scanning (TLS) with a very high scanning resolution of $1 \mathrm{~cm}$ at the medium distance of $100 \mathrm{~m}$ which is equivalent to an angular resolution of $2^{\prime \prime}$. In the two corresponding plots, we validated the proposed experiment by a cover-based evaluation method. The evaluation was realized by pixel-wise overlap comparison of estimated regeneration coverage and ground truth. All the TLS points between $1 \mathrm{~m}$ and $5 \mathrm{~m}$ height above the ground were first cropped out by visual inspection. Then, filtering was applied by a simple threshold to eliminate outliers (such as points below DTM) and stem points of overstory trees. A geo-referenced raster with a grid size of $3 \mathrm{~m}$ (the mean size of regeneration trees) was constructed to have a binary mask in which a grid cell indicates "true" if points of regeneration trees fall in it.

We tested the proposed method on 23 circular sample plots in the 2012 dataset with an area size of $500 \mathrm{~m}^{2}$ for each plot. From the 2011 dataset, a particular plot with an area of $919 \mathrm{~m}^{2}$, and from the 2009 dataset, another unique plot with an approximate area of $2450 \mathrm{~m}^{2}$ were available. Tree numbers (with diameter $>7 \mathrm{~cm}$ ) were calculated from the number of trees in each plot 
divided by the area in ha (hectare). Furthermore, the trees were divided into three layers with respect to the top tree height $h_{t o p}$ of the plots, where $h_{t o p}$ was defined as the average height of the 100 highest trees per ha (Heurich, 2008). From these layers, we assumed $66 \%$ of the top tree height $h_{t o p}$ belonged to the overstory. Then, the overstory density for each plot was defined as the relationship between tree numbers in $66 \%$ of the top tree height $h_{\text {top }}$ layer and the total number of trees in the plot. Note that the definition of the stem density (trees/ha) was limited to mature trees.

\subsection{Results}

\subsubsection{Regeneration coverage estimation}

The 3D segmentation was applied to the sample plots of each dataset separately. For the current experiment, we defined the standard control parameters for all the plots in advance. The control parameters for the mean shift were $2.4 \mathrm{~m}$ for the radius $h_{r}$ and $3.5 \mathrm{~m}$ for the kernel height $H$. The minimum number of points in a segment $N C u t_{\text {minpoints }}$ was set equal to 12 based on the field inventory and forest characteristics. The main control parameter of the normalized cut segmentation, the normalized cut threshold $N C u t_{\text {Thres }}$, was defined empirically as 0.23 . The parameters $\sigma_{h p}, \sigma_{v p}, \sigma_{I W}, \sigma_{M}$ were also assigned experimentally (see (Reitberger et al., 2009)). Fig. 3.5 summarizes the regeneration coverage estimated by the $3 \mathrm{D}$ segmentation for all the sample plots in the 2012 dataset with different overstory properties.

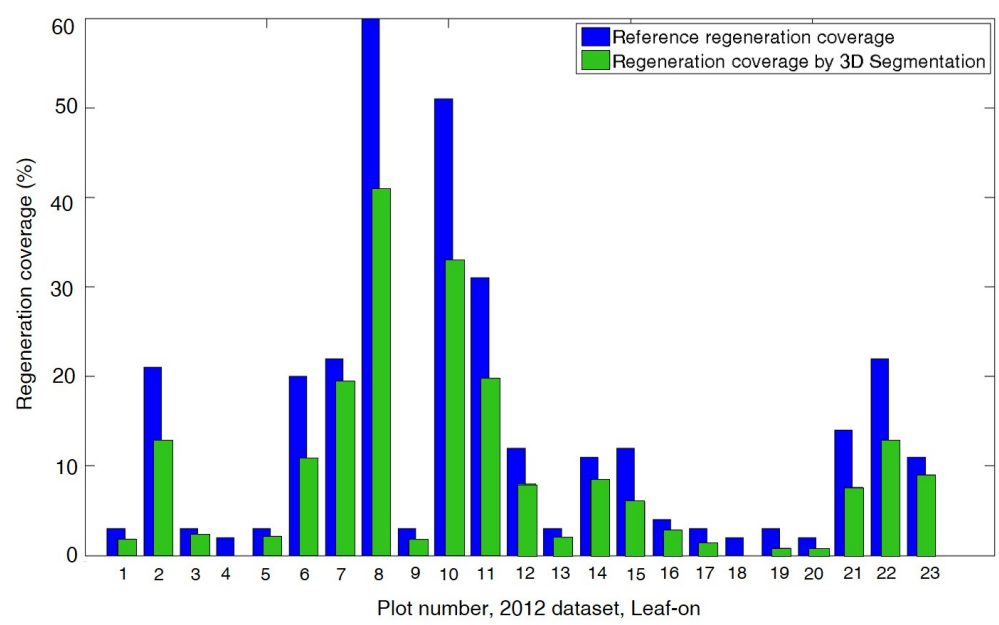

Figure 2.5: Estimation of regeneration coverage by 3D segmentation in the 2012 dataset vs. reference data.

Fig. 3.6a and Fig. 3.6b present the results of the 3D segmentation for the single plots in the 2011 and 2009 datasets. 

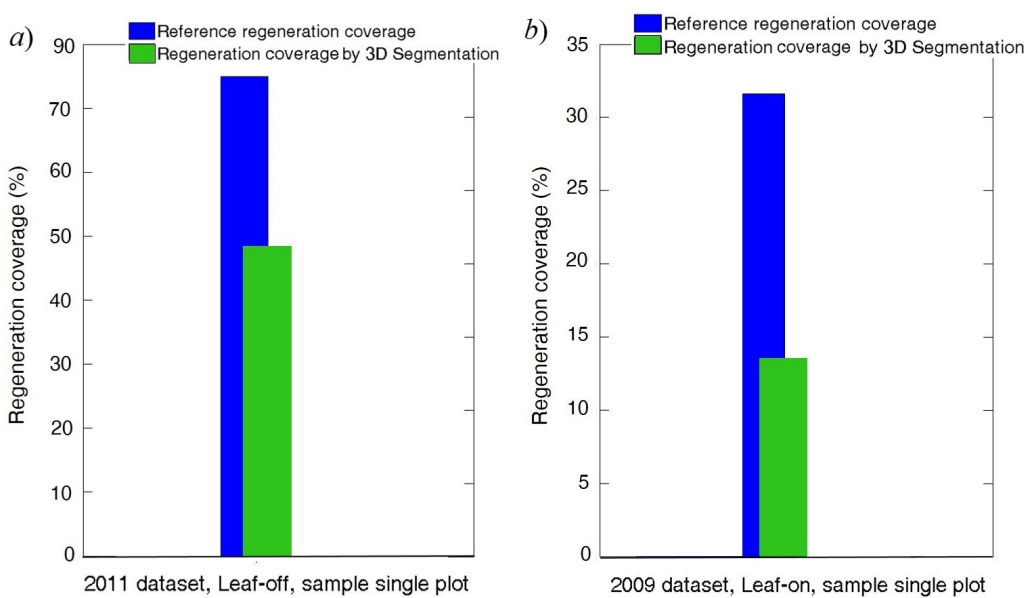

Figure 2.6: Estimation of regeneration coverage by 3D segmentation (a) in the 2011 and (b) 2009 datasets vs. reference data.

It can be seen in the plots with a high regeneration coverage (see the blue bars in Fig. 3.5), such as plot 7, plot 8, and the single plot from 2011 in Fig. 3.6a, we estimated more than $60 \%$ of the regeneration coverage correctly compared to the reference data. A subset of approximately $30 \%$ of the regeneration coverage could not be estimated. The highest accuracy belonged to plot 3 in the 2012 dataset, in which more than $70 \%$ of the regeneration coverage was estimated correctly compared to the reference data. As expected, we can see in Fig. 3.5, on average, the lowest estimation accuracy of approximately $1 \%$ belong to the plots with low regeneration coverage. On average, we achieved an underestimation of $8.3 \%$ using the proposed method for the estimated regeneration coverage.

A linear regression model in Fig. 3.7, using overstory density as the independent predictor variable, explained $62 \%$ of the variance associated with the accuracy of the estimated regeneration coverage.

In Fig. 2.8, the accuracy of the estimated regeneration coverage is also plotted against the deciduous tree species composition in the overstory. Different tree species (either deciduous or coniferous), had different contributions to the estimated regeneration coverage accuracy because of their structures and reflection properties. A linear regression model using deciduous tree species composition in the overstory as an independent predictor variable could explain $57 \%$ of the variance associated with the accuracy of the estimated regeneration coverage. However, as a further test, a linear regression model using coniferous tree species composition in the overstory as an independent predictor variable could explain $70 \%$ of the variance associated with the estimation accuracy of regeneration coverage.

The selected group of plots from the 2012 dataset with dominant coniferous tree species in the overstory showed a higher estimation accuracy compared to plots with dominant deciduous trees. 


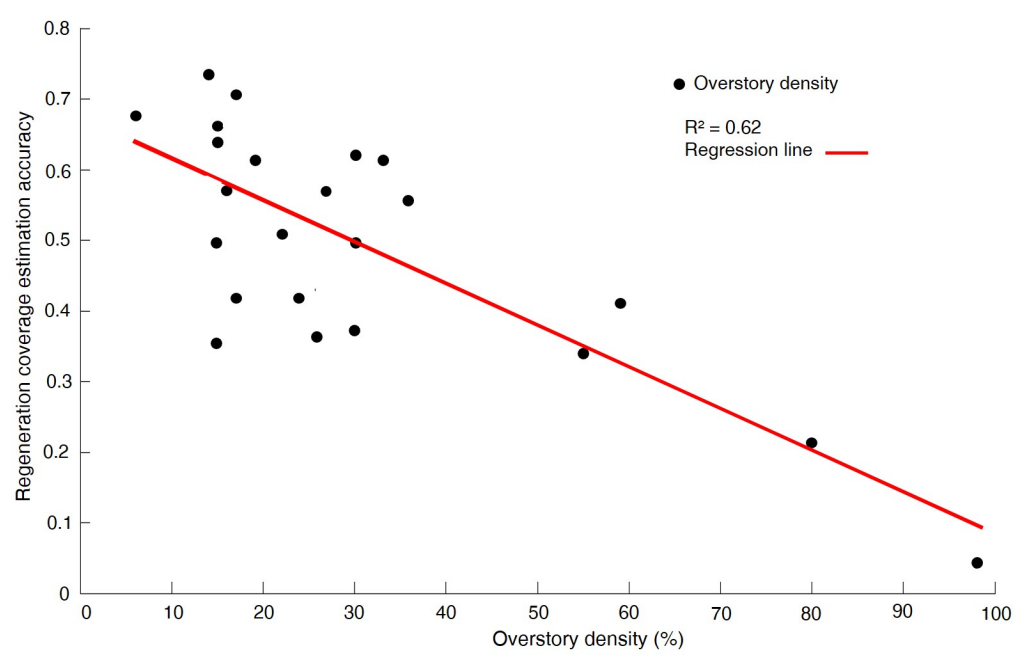

Figure 2.7: Effect of overstory density on the accuracy of estimated regeneration coverage.

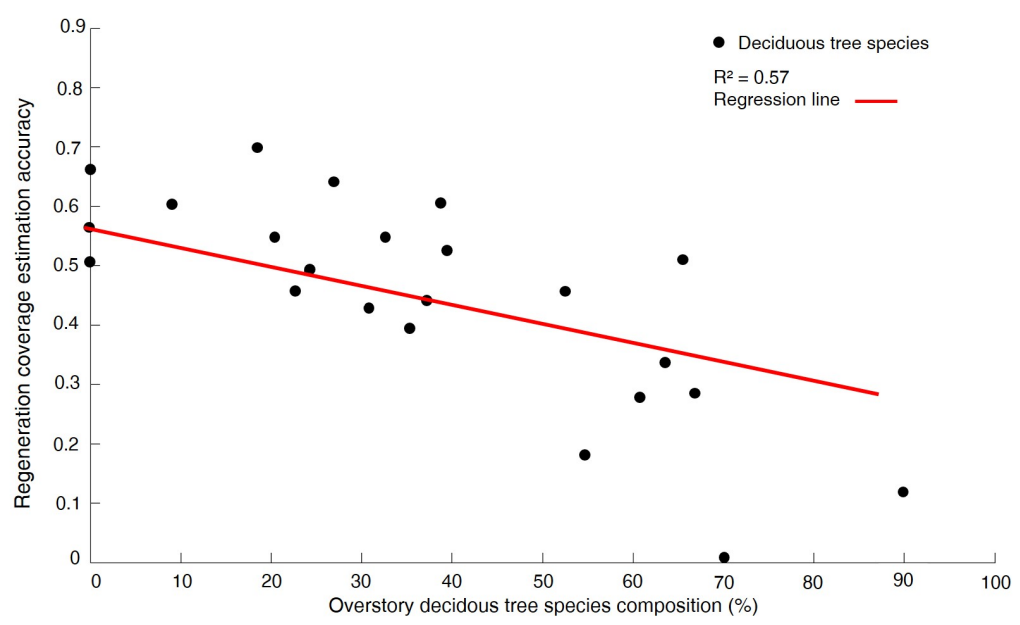

Figure 2.8: Effect of deciduous tree species composition in the overstory on the accuracy of estimated regeneration coverage.

\subsubsection{Sensitivity analysis}

The proposed method requires proper values for the kernel radius $h_{r}$ and height $H$ parameters of the mean shift technique and the control parameters of the normalized cut. We demonstrate the performance of the 3D segmentation with two control parameters of the normalized cut threshold NCut $t_{\text {Thres }}$ and the minimum number of points in a segment $N$ cut $t_{\text {min }}$ points. The results from the variation of the minimum number of points in a segment $N$ cut $t_{\text {min }}$ points values showed that there was no significant improvement to the accuracy 
of the estimated regeneration coverage. However, a larger normalized cut threshold $N C u t_{\text {Thres }}$ value led to more segments, which resulted in an overall higher completeness but lower correctness across the entire plot. This can be attributed to the fact that the larger normalized cut threshold $N C u t_{\text {Thres }}$ is a weaker stopping criterion for the bipartitioning process, since the segments have to be more similar to each other to break off the segmentation, thereby resulting in a larger quantity of smaller segments. The performances of the 3D tree segmentation algorithm for the selected plots were almost identical if a normalized cut threshold $N C u t_{T h r e s}$ value smaller than 0.2 was selected. Generally, the lower threshold values seemed to not play as significant a role as expected. Fig. 2.9 shows the sensitivity analysis with respect to the normalized cut threshold $N C$ ut $_{\text {Thres }}$ and the minimum number of points in a segment $N$ cut $t_{\text {min }}$ points parameters. We used the optimal values mentioned in Section 4.1 for the control parameters of the normalized cut.
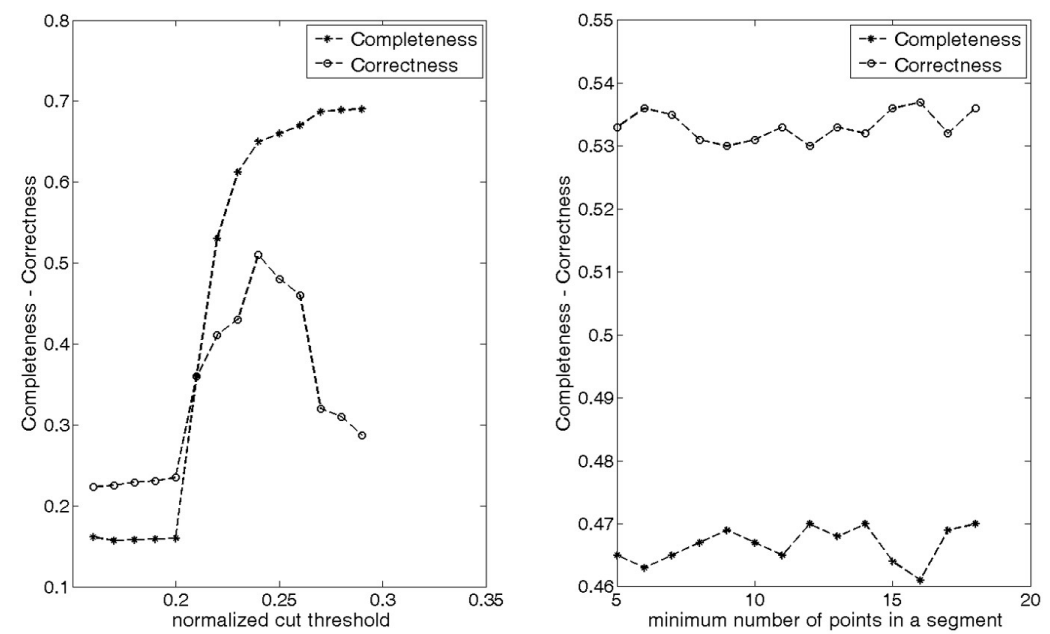

Figure 2.9: Sensitivity analysis of the normalized cut threshold NCut Thres $_{\text {S }}$ and the minimum number of points in a segment $N c u t_{\text {min }}$ points parameters.

As a further test, in Fig. 2.10a, for the 2012 dataset, we demonstrate the influence of the kernel radius $h_{r}$ variation with constant height $H$ on the $3 \mathrm{D}$ segmentation results by examining the RMSD values. The constant test value for kernel height $H$ was selected considering the mean height of trees with regeneration in the study area. Furthermore, in Fig. 2.10b, we demonstrate the sensitivity analysis for the kernel height $H$ where the value of the kernel radius $h_{r}$ and the other normalized cut control parameters during the analysis remained constant.

In Fig. 2.10 a small value for the kernel radius $h_{r}$ produced several distinct segments, while a large one aggregated small segments into larger ones. The minimum mean RMSD value from the sensitivity analysis of the regeneration coverage estimation by the $3 \mathrm{D}$ segmentation was 5.83 . Therefore, the kernel radius $h_{r}$ optimal value was equal to $2.4 \mathrm{~m}$ for the constant height $H$ of $4 \mathrm{~m}$. In Fig. 2.10b, based on previous studies for the kernel radius $h_{r}$, we selected 
a)

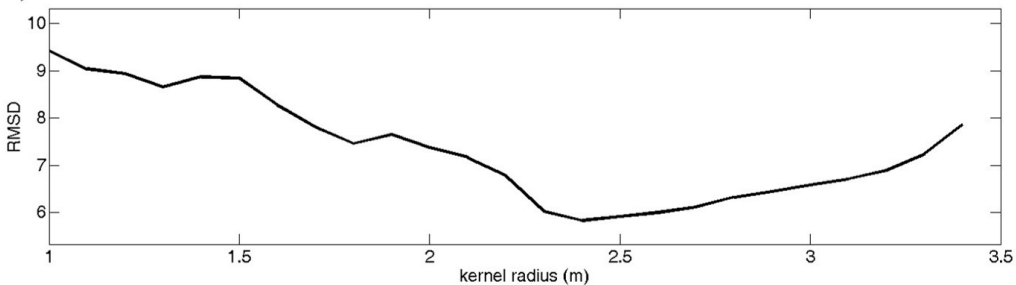

b)

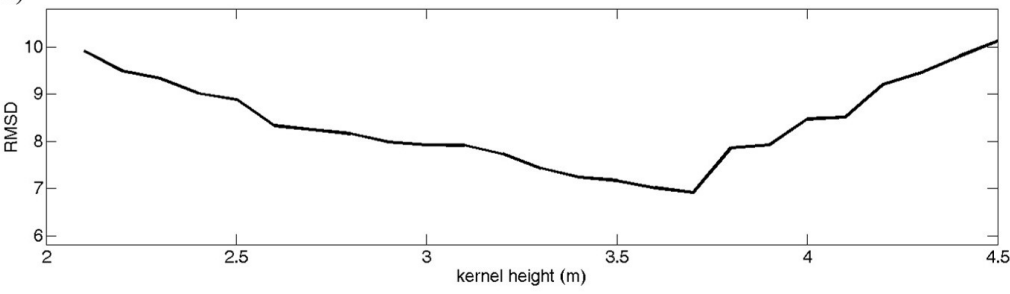

Figure 2.10: Sensitivity analysis for the 2012 dataset, (a) with respect to the mean shift kernel radius $h_{r}$ and $(H=4 \mathrm{~m})$; (b) with respect to the mean shift kernel height $H$ and $\left(h_{r}=2.4 \mathrm{~m}\right)$.

$2.4 \mathrm{~m}$ as the best estimation value. The results became degraded for the plots that had more challenging structures, such as high overstory density. Setting smaller values for the kernel height $H$ increased the RMSD rapidly, which reduced the accuracy of the $3 \mathrm{D}$ segmentation. Smaller values led to more underestimation in the regeneration coverage. In Fig. 2.10b, the minimum mean RMSD value for the regeneration coverage estimation by the $3 \mathrm{D}$ segmentation is 7.17. Thus, the optimal value for the kernel height $H$ was $3.5 \mathrm{~m}$ for the constant radius $h_{r}$ of $2.4 \mathrm{~m}$.

Because of the availability of TLS data as reference data for the 2009 and 2011 datasets, we performed a sensitivity analysis with correctness, completeness and $F_{1}$ score measures. Fig. 2.11 shows the sensitivity analysis results of the 2009 and 2011 datasets for the mean shift kernel radius $h_{r}$ and height $H$ parameters based on varying one parameter while keeping the other one constant.

The lower values for the kernel radius $h_{r}$ presented a higher estimation accuracy. However, this led to a larger overestimation. For the kernel height $H$, a low value led to underestimation in the regeneration coverage by the 3D segmentation, whereas high values, even with higher estimation accuracy, contained a high number of neighboring tree segments, which led to the overestimation. Also, the best $F_{1}$ score value for the optimal kernel parameters in the 2009 dataset was 0.43 for the kernel height $H$ and 0.6 for the kernel radius $h_{r}$. The test for the 2011 dataset achieved the highest $F_{1}$ score value of 0.64 for the kernel radius $h_{r}$ and 0.6 for the kernel height $H$ respectively. Based on the sensitivity analysis, we selected $2.4 \mathrm{~m}$ for the kernel radius $h_{r}$ in all sample plots and $3.5 \mathrm{~m}$ for the height $H$, where the higher estimation accuracy for regeneration coverage and relatively lower 
overestimation was available. In our method, complete tree objects could not be extracted from the mean shift step. The provided clusters were incomplete, and they had to be merged by the normalized cut algorithm. Therefore, we applied the optimal values for the mean shift control parameters instead of adaptive values.

\subsection{Discussion}

This study followed the two main goals of (1) assessing the regeneration coverage estimated by the $3 \mathrm{D}$ segmentation in a multilayered temperate forest and (2) comparing the estimation accuracies, which were affected by the different overstory densities and tree species composition.

The results showed that the average over- and understory vegetation was correlated with the height distribution. It could be used to estimate the regeneration coverage, if confined to the height below $5 \mathrm{~m}$. Moreover, the 3D segmentation method estimated, on average, $60 \%$ of the regeneration coverage in the temperate forest compared to the reference data (Fig. 3.5 and 3.6). However, the overstory in the leaf-on situation was the main factor in preventing the laser beam from reaching the understory. Therefore, in the plots with up to $2 \%$ regeneration coverage, the $3 \mathrm{D}$ segmentation was not successful in constructing the $3 \mathrm{D}$ regeneration segments.

The correlation between the reference data and the 3D segmentation results depended on the forest type and tree species composition in the overstory. The results in Fig. 3.7 revealed that the average regeneration coverage estimation accuracy in the understory was highly correlated with the overstory density. In addition, the complex canopy and mixed branches reduced the accuracy of the estimated regeneration in the 3D segmentation approach, especially in deciduous forests such as plots 15 and 19 in the 2012 dataset. In leaf-on conditions, an increase of $10 \%$ in the overstory density decreased the estimation accuracy of the regeneration coverage by about $6 \%$. In previous studies, variations in the overstory has been shown to have a direct effect on the presence and density of the understory (Latifi et al., 2015b; McKenzie et al., 2000). Furthermore, every $10 \%$ increase in the deciduous tree species composition in the overstory decreased the estimation accuracy of the regeneration coverage by about $4 \%$. However, in the selected group of plots, a $10 \%$ increase in the coniferous tree species distribution unexpectedly increased the estimation accuracy by about $12 \%$. This could be attributed to the geometry of the coniferous trees with less complex branches. The highest correlation between the $3 \mathrm{D}$ segmentation results and the reference data belonged to the plots with lower overstory density and higher percentage of coniferous trees. The main problem of the proposed method in plots with dominant deciduous tree species was to correctly estimate the regeneration coverage when only a small fraction of the laser beam can penetrate through the overstory. The estimation accuracy in the selected plots was highly correlated with the presence of deciduous trees of the overstory, since the lowest estimation accuracies belonged to the plots with high overstory density and dominant deciduous trees, such as plots 18 and 20 in the 2012 leaf-on dataset. Another 

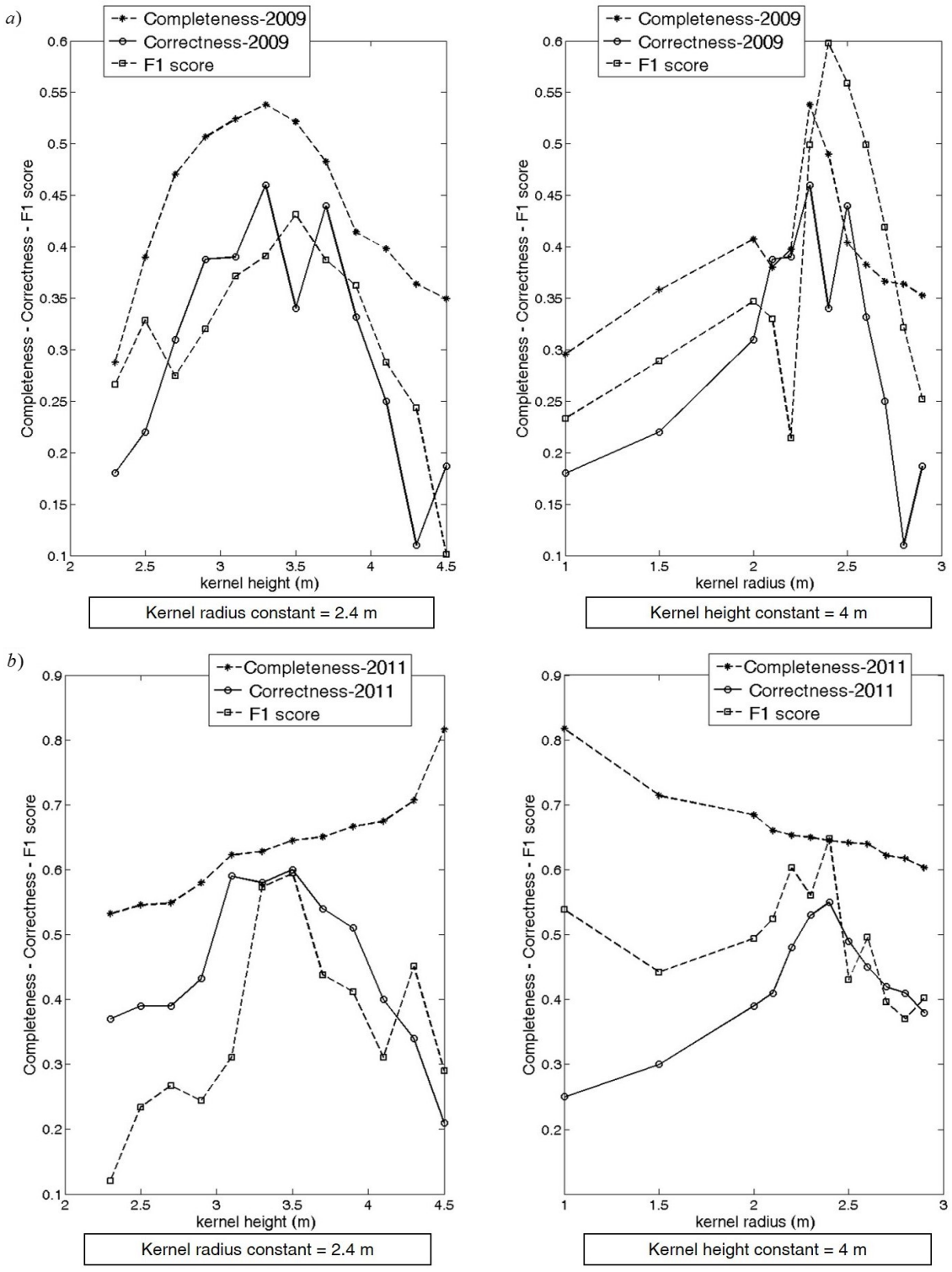

Figure 2.11: Sensitivity analysis of the mean shift kernel parameters as Completeness, Correctness and $F_{1}$ score (a) for the 2009 and (b) 2011 datasets.

problem could be related to the reference data collection. In the understory, an estimation of regeneration coverage within a complex plot had difficulty distinguishing the borders between ground vegetation and regeneration in a $3 \mathrm{D}$ space and predicting the coverage accurately.

The experiment was based on the three full waveform ALS datasets from various sample plots in the study area. We were not able to perform a multitemporal analysis for the estimated regeneration coverage of the available 
datasets, since they were not acquired from the same sample plots. In the available datasets, we did observe the estimated regeneration coverage by $3 \mathrm{D}$ segmentation was almost independent of leaf-on and leaf-off conditions in the forest. However, the penetration was slightly different in deciduous and coniferous stands. Moreover, in leaf-on conditions, the crown of overstory deciduous trees were more abundant, which led to stronger spatial evidence of their difference from coniferous trees. It is clear from Fig. 3.5 and 3.6 that the short trees in the multilayered temperate forest have a greater potential to be better resolved by the $3 \mathrm{D}$ segmentation method. However, points of tree crowns and branches that actually belong to neighboring trees weaken the similarity function in the graph-based segmentation, leading to the lower accuracy of the estimated regeneration coverage in the understory.

From the current experiment, we conclude that the 3D segmentation was able to estimate regeneration coverage successfully in the forest understory. In the context of regeneration coverage, studies such as (Wing et al., 2012) reported an overall accuracy of $\pm 22 \%$ using a density metric of ALS point cloud to estimate aggregated understory elements, including tree regeneration. Our results are somewhat inferior (approximately 30\% underestimation), although our scenario was more complex since the aforementioned study was carried out in a single tree species forest, and different understory components (regeneration, shrubs etc.) were not distinguished. Furthermore (Yao et al., 2013) reported an enhanced scheme for detecting single trees in lower forest layer. Our study confirms that their proposed methodology can be successfully adapted for the estimation of regeneration coverage by incorporating the influence of tree species composition and compensating for the effect of overstory density. To render the results more transferable, ALS data should be acquired from the equivalent sample plots during leaf-on and leaf-off conditions.

\subsection{Conclusions}

This study focused on the regeneration coverage estimation in a multilayered temperate forest by applying adapted 3D segmentation to full waveform ALS data. Unlike other methods, our approach did not rely on a CHM, and was directly applied to the $3 \mathrm{D}$ point clouds. We did not try to explain the tree species composition in the analysis; this will constitute a future research topic. The 3D segmentation method was able to correctly estimate up to $70 \%$ of the regeneration coverage in the multilayered temperate forest (as compared to the reference data). Our experiment showed that the accuracy of the regeneration coverage estimation was strongly correlated to forest characteristics such as overstory density and tree species composition. Moreover, the accuracy of the regeneration coverage estimation was negatively impacted by the higher density of deciduous tree species in the overstory.

Further improvements to the method would be achieved by extending the $3 \mathrm{D}$ segmentation with a classification of regeneration into deciduous and coniferous tree species in order to assess the influence of tree species on the regeneration estimation results. According to previous studies, the mean pulse 
intensities and tree canopy properties were clearly different for coniferous and deciduous trees. 



\section{Detection of single tree stems in forested areas from high density ALS point clouds}

This chapter is based on:

Amiri, N., Polewski, P., Yao, W., Krzystek, P., Skidmore, A., 2017. Detection of single tree stems in forested areas from high density ALS point clouds using $3 \mathrm{~d}$ shape descriptors. ISPRS Annals Photogram., Remote Sens. Spatial Inform. Sci. 3542. 


\section{Abstract}

Airborne Laser Scanning (ALS) is a widespread method for forest mapping and management purposes. While common ALS techniques provide valuable information about the forest canopy and intermediate layers, the point density near the ground may be poor due to dense overstory conditions. The current study highlights a new method for detecting stems of single trees in $3 \mathrm{D}$ point clouds obtained from high density ALS with a density of 300 points $/ \mathrm{m}^{2}$. Compared to standard ALS data, due to lower flight height $(150-200 \mathrm{~m})$ this elevated point density leads to more laser reflections from tree stems. In this work, we propose a three-tiered method which works on the point, segment and object levels. First, for each point we calculate the likelihood that it belongs to a tree stem, derived from the radiometric and geometric features of its neighboring points. In the next step, we construct short stem segments based on high-probability stem points, and classify the segments by considering the distribution of points around them as well as their spatial orientation, which encodes the prior knowledge that trees are mainly vertically aligned due to gravity. Finally, we apply hierarchical clustering on the positively classified segments to obtain point sets corresponding to single stems, and perform $\ell_{1}$-based orthogonal distance regression to robustly fit lines through each stem point set. The $\ell_{1}$-based method is less sensitive to outliers compared to the least square approaches. From the fitted lines, the planimetric tree positions can then be derived. Experiments were performed on two plots from the Hochficht forest in Oberösterreich region located in Austria. We marked a total of 196 reference stems in the point clouds of both plots by visual interpretation. The evaluation of the automatically detected stems showed a classification precision of 0.86 and 0.85 , respectively for Plot 1 and 2 , with recall values of 0.7 and 0.67 . 


\subsection{Introduction}

Accurate measurements of forest structure are increasingly required across large areas to support a wide range of activities related to sustainable forest management. Cost-effective and more automated methods are needed to provide tree attribute data for forest ecosystem services. Therefore, remote sensing tools are increasingly being used to survey forest structures. However, the spatial extent and spatial resolution of a given sensor are inversely related. Airborne Laser Scanning (ALS) has become a key tool for gathering information on 3D structure of forests (Wulder et al., 2012). The derived information from ALS data can grant detailed estimations of forest characteristics and single tree analysis (Yao et al., 2012; Maltamo et al., 2012). Previous studies are showed several properties of single trees such as species, height and crown properties which can be measured with high resolution ALS data (Maltamo et al., 2012). However, limited persistence has been done on the stem detection of single trees. For instance, single tree stems have been determined from the interpolated CHM (Canopy Height Model) at the highest positions(Solberg et al., 2006) or by using hierarchical clustering for stem reflections and reconstructions with a RANSAC-based adjustment (Reitberger et al., 2007). Due to the low point density and lack of information about the reflection characteristics, minor focus has been given to tree reconstruction using laser hits on the stems (Reitberger et al., 2007; Polewski et al., 2016). In order to detect stem of trees, data with greater precision would be required to allow a more accurate representation of the actual discontinuities in the single trees (Vauhkonen, 2010).

On the other hand, Terrestrial Laser Scanning (TLS) has also been proven to be a suitable method for obtaining very detailed information about geometry of trees in forests (Liang and Hyyppä, 2013). Pfeifer and Winterhalder (2004) showed a method for reconstructing the cross section of tree stems and branches from TLS data with free-form curves. However, the study indicated that expected cross section reconstruction works only satisfyingly, if the branch is covered with points from all sides which it is not possible due to occlusions. Liang et al. (2012) presented a fully automatic algorithm based on single-scan TLS data for stem detection and mapping with the overall accuracy of $73 \%$. The stem points are established using classification based on the local covariance matrix features. The provided method is capable of giving good parameters only when the points are evenly distributed on the tree trunk; however the applicability of feature estimation for a group of points has not been taken into account. Lindberg et al. (2012) projected candidate stem points onto a 2D plane. They applied Hough transforms to locate circles, representing the potential stems. Heinzel and Huber (2016) used a 3D voxel grid transformation of the input TLS point clouds to detect tree stems using morphological operations and empirical rules. They reported detection rates of $84 \%$ to $97 \%$ for the number and location of stems depending on the tree DBH (Diameter at Breast Height). Olofsson et al. (2014) by voxelizing the point cloud and analyzing the influence of different height layers could estimate tree stem positions with an average proportion of $87 \%$. Wang et al. (2016) performed in the first step, RANSAC based circle fitting of projec- 
ted stem points, which is later followed by RANSAC cylinder fitting in 3D space. Polewski et al. (2017) proposed a statistical framework for detecting cylinders based on accumulation and voting in parameter space. The size of the accumulation cell is determined automatically using bandwidth selection methods for kernel density estimators, which relaxes the requirement of setting this critical parameter manually or through trial-and-error. The method is applied on a dense 3D point cloud for mapping fallen tree stems. Based on the mentioned studies, the main advantage of TLS data lies in its capacity to scan a sample plot in forest horizontally and vertically in detail. However, important factors such as the occlusion effect and relatively high cost of the instrument transportation from site to site are negatively effecting the use of TLS data. Moreover, the co-registration of several scans covering a study area is an essential step in the interpretation of multi-scan data acquisition. The fully automated registration between several scans at the point level is still challenging.

In standard operational applications of ALS, the flight heights are usually between 400 to $800 \mathrm{~m}$, resulting in point densities up to 30 points $/ \mathrm{m}^{2}$. However, an alternative scenario is also possible, where the flying altitude is significantly decreased to below $150 \mathrm{~m}$, for example using a helicopter mounted system or even a UAV with around $50 \mathrm{~m}$ flight height. This can be seen as a middle ground between standard ALS and TLS techniques, trading off large area coverage for increased point density. This tradeoff is due to the lower flight altitudes associated with this technique compared to standard ALS campaigns, which results in smaller footprints. Razak et al. (2011) used high resolution DEMs (Digital Elevation Models) extracted from high density ALS data to semi-automatical recognition of morphometric landslide features even under forest canopy. Höfle et al. (2012) provided an example of high density ALS data potential to use for urban vegetation detection purposes. They used a high point density of 50 points $/ \mathrm{m}^{2}$. Khosravipour et al. (2014) also presented an algorithm which is able to create a pit-free CHM raster using full waveform ALS data with 160 points $/ \mathrm{m}^{2}$ density. The algorithm significantly improves the accuracy of tree detection compared to local maxima based methods. The data collected by high density ALS systems is less precise in comparison with TLS. However, within an equal time frame, the area that can be investigated by utilizing high resolution ALS is significantly larger than the area investigated with TLS. Also the aforementioned TLS based methods for stem detection are not practical for the applications using ALS data, since the curvature shape of the tree trunk in ALS point clouds is not captured as detailed as in case of TLS. On the other hand, the increased point density, resulting from lower flight height can provide more details in the point clouds compared to standard ALS, enabling the use of 3D spatial descriptors to locate individual tree stems. Therefore, an automated method for stem mapping within high-density ALS data is interesting from forestry application point of view.

The objectives of this study are to develop a new method for single tree stem detection based on high density ALS data using (i) point and object part level $3 \mathrm{D}$ shape descriptors, and (ii) $\ell_{1}$ regularized line fitting with a prior on orientation. A further objective is (iii) to assess the accuracy of 
detected tree stems. This paper is motivated by the successful application of high density ALS systems for precise monitoring of vegetation and forest structure, reported in the aforementioned studies. Also, detected tree stems could be used to improve the 3D segmentation algorithm as prior knowledge in terms of the detection rate and the position of trees.

The remainder of this work is structured as follows: Section 2 focuses on the details of our approach. Section 3 shows the experiment results. Finally, the results are discussed with conclusions in Sections 4 and 5.

\subsection{Method}

We adapt the method of Polewski et al. (2015b) which is originally designed for fallen tree segmentation, to detect the standing stems of single trees from unstructured high density ALS point clouds. The main goal is to detect linear structures in the ALS 3D point clouds which are likely to represent single tree stems. The output of our method is a set of 3D lines which correspond to detected stems. The pipeline describing our approach is presented in Fig. 3.1. Our approach is a three-tiered detection procedure at (i) point, (ii) segment and (iii) object levels. The segment term refers to the grouping of points within a fixed length cylindrical neighborhood which are likely to represent part of a tree stem. Objects refer to entire tree stems which are composed from groups of similarly aligned segments. The method proceeds as follows. First the likelihood of points belonging to a tree stem is estimated. Second, the segments containing the highest probability stem points are detected in the 3D point clouds. Finally the segments are merged through hierarchical clustering to produce single tree stems. In the following, we explain the steps of our method in detail.

\subsubsection{Point level}

In the input data depending on the forest characteristics, different objects are present such as ground vegetation, regenerations, standing tree stems etc. The focus of this step is to obtain for every point, an estimate of the probability that it belongs to a tree stem. High density ALS data can provide a range of features related mainly to the $3 \mathrm{D}$ structure of single trees. The features derived from 3D point clouds can be grouped into three categories:

1. Point feature histograms (PFH): a local 3D shape descriptor of the neighborhood around the target point, based on the angular relationships between adjacent surface normals. It is useful for distinguishing between different types of surface classes based on their shape (plane, cylindrical, spherical, etc) (Rusu et al., 2008) (Rusu et al., 2008; Polewski et al., 2015b).

2. Covariance features: set of features derived from eigenvalues of local neighborhood covariance matrix around target point (see Weinmann et al. (2015a)).

3. Normalized height: the relative point heights over the Digital Terrain Model (DTM). 


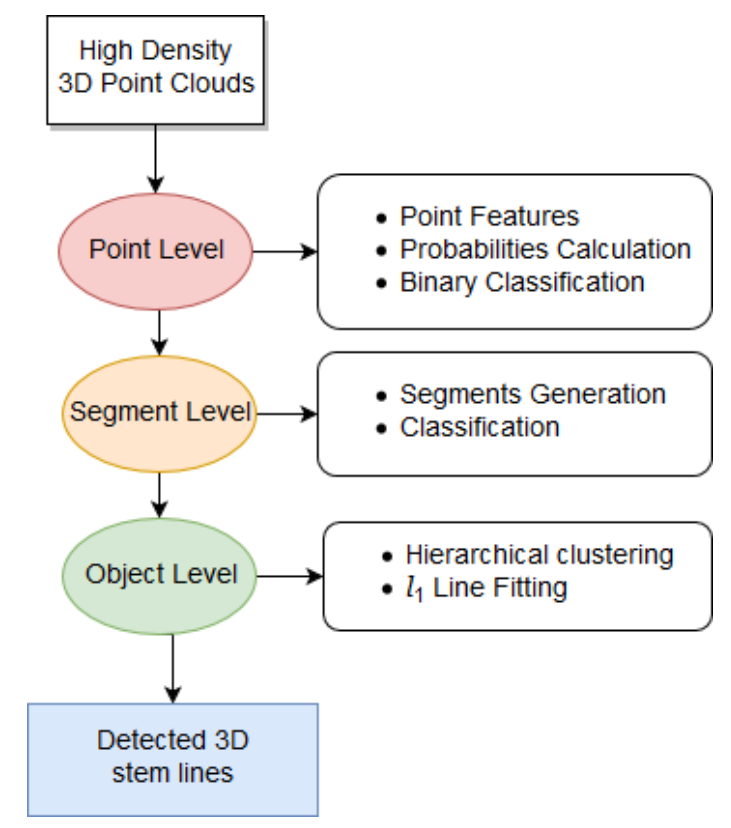

Figure 3.1: Overview of stem detection pipeline.

For the point classification level we chose Random Forest (Breiman, 2001) as a binary classifier due to its robust ability to estimate the class probability.

\subsubsection{Segment level}

This level focuses initially on generating segment candidates. For each point with high probability, a cylindrical neighborhood with constant radius $r_{\text {seg }}$ and height $l_{\text {seg }}$ is defined. Afterwards, all the points inside the cylinder space are taken into account to perform the least-squares Orthogonal Distance Regression (ODR) (Al-Subaihi and Watson, 2004).This is done by eigenanalysis of the point coordinates' covariance matrix: the ODR line's direction is the eigenvector corresponding to the maximum eigenvalue, and this line passes through the points' centroid. The ODR line becomes the segment's axis.

We classify the candidate segments generated in the previous step into the 'positive' and 'negative' groups. The 'positive' group represents the segments which are really parts of tree stems, and the 'negative' contains branches, ground vegetation, and etc. The first set of segment features are derived from a modified version of Cylindrical 3d Shape Context (CSC) built around the segments' axes (Polewski et al., 2015b) (see Fig. 3.2). A spatial distribution histogram of points within the cylindrical volume around the segment axis can then be constructed. The point counts inside the histogram bins form the features for the classification.

The second set of features are calculated based on the angular deviation of the 


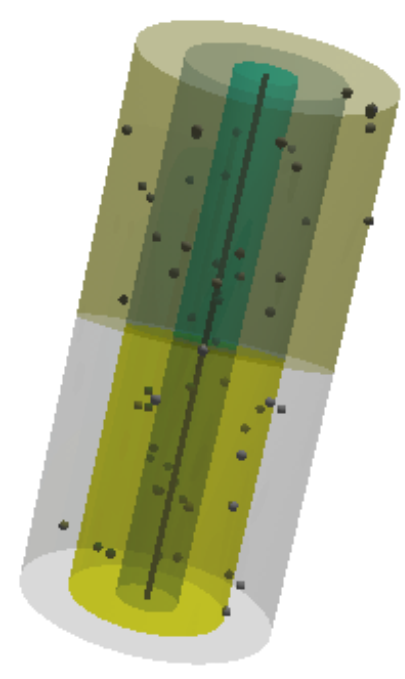

Figure 3.2: Cylindrical 3D Shape Context around a segment.

segment axes from the world $Z$. The segments with the deviation larger than $\theta_{t h r}$ are rejected without regard to remaining feature values. Additionally, the point probability statistics are extracted as another group of features for classification. In the current study, we looked at the points inside the segment neighborhood and we created the stem point probability histograms quantized at 0.2 intervals, resulting in 5 features.

\subsubsection{Object level}

The goal of current level is to take the stem segments with high probability from the previous step and merge them to reconstruct individual tree stems. This is based on the collinearity and mutual distances between segments.

\subsubsection{Hierarchical clustering}

In the next step of the pipeline, the representative 'positive' segments are merged together. For this purpose, first we have a combination of two distances to calculate. The aggregate distance $d$ between segments $S_{i}$ and $S_{j}$ is the weighted sum of angular deviation $d_{A}$ and the spatial distance between point centroids $d_{C}$ (see Eq. 3.1).

$$
d\left(S_{i}, S_{j}\right)=d_{A}\left(\vec{S}_{i}, \vec{S}_{j}\right)+w_{1} \cdot d_{C}\left(\bar{S}_{i}, \bar{S}_{j}\right)
$$

In the Eq. 3.1, $\vec{S}$ refers to the axis and $\bar{S}$ indicates the point centroid of each segment. $w_{1}$ is the weight component for the spatial distance $d_{C}$. Fig. 3.3 
shows the angular deviation $d_{A}$ and the spatial distance $d_{C}$ between two segments surrounded by cylinders.

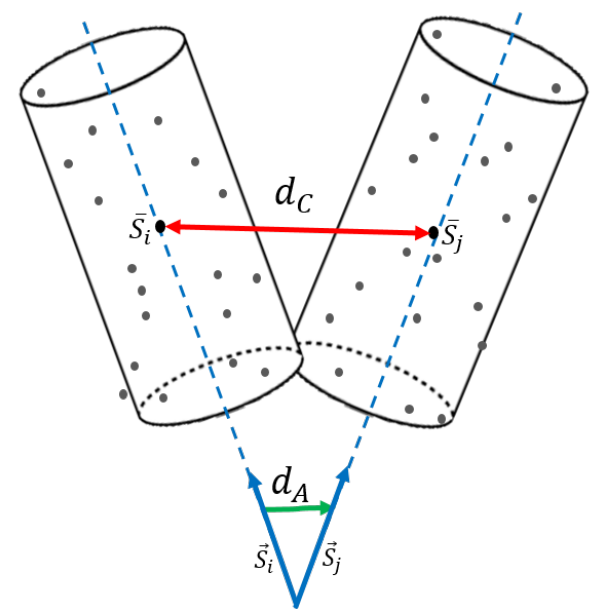

Figure 3.3: The aggregate distance $d$ between two segments; the angular deviation $d_{A}$ is shown with green arrow and the spatial distance between point centroids $d_{C}$ with red arrow.

The following hierarchical clustering scheme (Van Der Heijden et al., 2005) is applied to merge 'positive' segments based on the aggregate distance matrix $d$ explained above.

1. Assign each point to its own cluster.

2. Find the closest pair of clusters which do not trigger the stopping criterion and merge them into one. The number of clusters reduces by one.

3. Compute the distance $D$ between the new cluster and each of the old clusters.

4. Repeat steps 2 and 3 until no more clusters can be merged under the stopping criterion.

In the current clustering process the distance $D$ between two clusters $C_{i}$ and $C_{j}$ is defined as the largest distance $d$ from any combination of member segments. The stopping criterion consists of two conditions. First, when considering two clusters $C_{i}, C_{j}$ if the spatial distance $d_{C}$ between any pair of segments $S_{k} \in C_{i}, S_{l} \in C_{j}$ is bigger than a predefined threshold $d_{C, \text { max }}$ then the merging of $C_{i}$ and $C_{j}$ is aborted. On the other hand, the ODR line is fitted to the set of points belonging to segments in $C_{i}, C_{j}$ and the orthogonal projected distance of all points to the ODR line is calculated. If the projected distance of any point is greater than a set threshold $d_{p, \max }$ or the angular deviation between the fitted line and the $Z$ axis exceeds $d_{A, \max }$, this also terminates the merging for that cluster pair. Fig. 3.4 represents the stopping criterion in the hierarchical clustering phase between segments. 


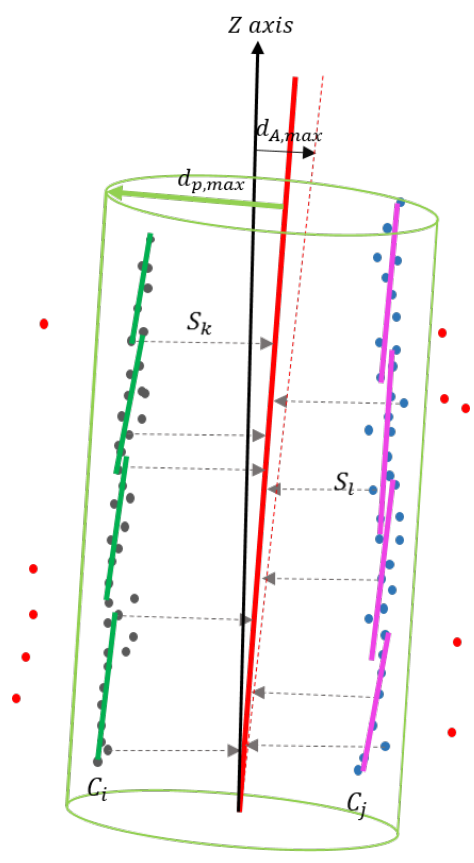

Figure 3.4: The stopping criterion for clustering the segments. The green lines represent the segments in cluster $C_{i}$ and the magenta lines show the segments in cluster $C_{j}$, respectively. The red line is the line fitted to both clusters' points. The red points outside the cylinder have projected distance greater than $d_{p, \max }$.

\subsubsection{Stem line fitting}

In the final step, for each cluster extracted from the merging step, containing all the stem points, we execute the line fitting procedure. For the fitting, we apply orthogonal distance regression with the $\ell_{1}$ norm of residuals as the error criterion and a prior on the line's verticality. The problem is formulated in terms of an energy minimization. The total energy for a line $L, E_{t o t}(L)$ is presented in the Eq. 3.2. The total energy $E_{t o t}$ has 2 components, the data goodness-of-fit term $E_{d}$ (Eq. 5.6) and the angular prior term $E_{a}$ (Eq. 3.4). The $\alpha$ element refers to the balance coefficient between energy terms. The angular prior was considered due to the prior knowledge that the tree stems grow almost always vertically. Furthermore, the $\ell_{1}$ norm is a more robust estimator and less sensitive to outliers available in the data compared to the $\ell_{2}$ norm (Al-Subaihi and Watson, 2004). In our experiment, the $\ell_{2}$ norm in the segment level and the $\ell_{1}$ norm is used in the object level, respectively. This decision is related to the computational expenses of the $\ell_{1}$ and $\ell_{2}$ fittings. Usually, the number of the segments to process is several orders of magnitude higher compared to the stems obtained from hierarchical clustering. On the other hand, the $\ell_{1}$ version is much more computationally expensive than the $\ell_{2}$ 
based method, which makes it intractable to apply for all segments.

$$
\begin{aligned}
& E_{\text {tot }}(L)=E_{d}(L)+\alpha E_{a} \\
& E_{d}(L)=\sum_{i}\left\|L\left(P_{i}\right)-P_{i}\right\|_{2} \\
& E_{a}(L)=\left|\arccos \frac{Z . w}{|Z| \cdot|w|}\right|
\end{aligned}
$$

In the Eq. 5.6, element $P_{i}, i \in 1 . . n$ refers to the $i$ th point inside the cluster. Furthermore, $Z$ element in the Eq. 3.4 represents $Z$ axis of world coordinate system. $L(P)$ term is the projection of point $P$ onto line $L$ and $w$ indicates $L$ 's direction vector. The line $L$ is parametrized using 4 values $a=\left[a_{1}, \ldots, a_{4}\right]$ (Al-Subaihi and Watson, 2004) showed in the Eq. 3.5:

$$
L(a, t)=\left[\begin{array}{c}
a_{1} t+a_{2} \\
a_{3} t+a_{4} \\
t
\end{array}\right]
$$

where $t$ is the location parameter. Note that the $z$ component of $w$ is always positive with a value of 1 , and therefore the angle between the world $Z$ axis and $w$ always lies in the interval $\left[0 ; \frac{\pi}{2}\right]$. This allows us to drop the absolute value on the angular prior term $E_{a}$ since the arccosine is guaranteed to be positive in the mentioned interval.

For the optimization of the orthogonal distance fitting, a two-step method similar to Liu and Wang (2008) as well as Watson (2002) is used. The first step for the re-parametrization computes the projection of all fitted points $P_{j}$ onto the current line $L(a, t)$ to minimize the distance from $P_{i}$ to $L$ :

$$
\min _{t_{j}}\left\|L\left(a, t_{j}\right)-P_{j}\right\|, \quad j=1, \ldots, n
$$

The line positions $t_{j}$ corresponding to the orthogonal projection onto $L$ can be recovered using Eq. 3.7, where $p_{0}=\left[a_{1}, a_{3}, 0\right]$ :

$$
t_{j}=\frac{\left(P_{j}-p_{0}\right) \cdot w}{w \cdot w}
$$

In the second step, we minimize the energy similar to $E_{t o t}$, but with the projected line positions fixed at $\left\{t_{j}\right\}$, with respect to shape parameters only, i.e.:

$$
\min _{a} \sum_{i}\left\|L\left(a, t_{i}\right)-P_{i}\right\|_{2}+\alpha E_{a}(a)
$$

For minimizing the objective (Eq. 3.8) the BFGS quasi-Newton method is applied (Wright and Nocedal, 1999). The derivative of the energy's data term 
with respect to any parameter $a_{k}$ is expressed as follows $\left(r_{j}=P_{j}-L\left(a, t_{j}\right)\right.$ is the $j-t h$ residual):

$$
\frac{\partial E_{d}(a)}{\partial a_{k}}=-\sum_{j} \frac{r_{j}}{\left\|r_{j}\right\|}\left\{\frac{\partial p_{0}}{\partial a_{k}}+t_{j} \frac{\partial w}{\partial a_{k}}\right\}
$$

By splitting the computation into two steps and fixing the $t_{j}$ values, the problem is considerably simplified, because otherwise the terms $t_{j}$ in Eq. 3.9 would depend on $a_{k}$, leading to the necessity of calculating the derivative of the product $\partial\left[t_{j}(a) \cdot w(a)\right] / \partial a$. Therefore, the derivatives with respect to the entire parameter vector are:

$$
\nabla_{a} E_{d}(a)=-\sum_{j} \frac{r_{j}^{T}}{\left\|r_{j}\right\|}\left[\begin{array}{cccc}
t_{j} & 1 & 0 & 0 \\
0 & 0 & t_{j} & 1 \\
0 & 0 & 0 & 0
\end{array}\right]
$$

As for the angular deviation term $E_{a}$, only the axis parameters $a_{1}, a_{3}$ have a non-zero derivative, given by:

$$
\nabla_{a_{1}, a_{3}} E_{a}(a)=\left[\begin{array}{l}
a_{1} \\
a_{3}
\end{array}\right] \frac{1}{\sqrt{1-\frac{1}{\|w\|^{2}}}\|w\|^{3}}
$$

We iteratively execute steps 1 and 2 in sequence until convergence is reached. Due to the non-convexity of the optimization problem, local optima may exist. To remedy that, we perform multiple restarts of the optimization with randomly initialized starting line hypotheses, and pick the lowest-energy solution. This yields the final fitted lines representing the detected single tree stems for all clusters.

\subsection{Experiments}

\subsubsection{Materials}

Experiments were conducted in the Hochficht forest close to Holzschlag in Oberösterreich region which is located in Austria. The study area is a type of mountainous forest with a high structural complexity, dominated by Norway spruce (Picea abies), European beech (Fagus sylvatica) and Silver fir (Abies alba). Two sample plots with the approximate area size of $6844.7 \mathrm{~m}^{2}$ and $17907 \mathrm{~m}^{2}$ and a mean tree density of 272 trees/ha were selected in the mixed forest to construct the experiment. The high density ALS data was acquired in leaf-on condition by the FMM GmbH Company with the VUX-1 scanner integrated in the VP-1 pod in September 2015 with an average point density of 300 points $/ \mathrm{m}^{2}$. We assumed that the data is given in a georeferenced coordinate system. The flying altitude between 150-200 m resulted in an average footprint size of $88 \mathrm{~mm}$. Fig. 3.5 shows a sample scene in the 3D point clouds associated with the visible single tree stems 


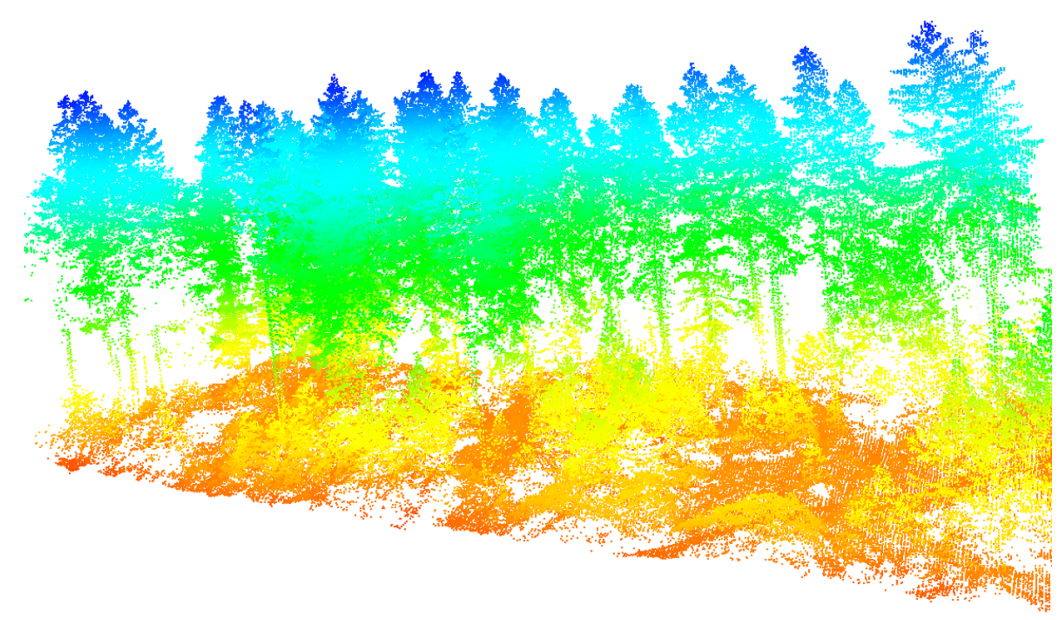

Figure 3.5: A point cloud visualization of sample forest scene with multiple visible stems (point clouds of scene colored by height over DTM).

\subsubsection{Training classifier}

We used parts of test plots and two additional plots to train the classifier in the point and segment levels. In the available 3D point clouds, a significant percentage of the stems is not at all represented (specially for the deciduous trees). For every single tree with visible stem, the points and segments were manually marked and assigned either the stem or the non-stem class by visual interpretation, resulting in training sets for binary classification. The total number of the marked points and segments were 20000 and 564, respectively. This represented about $28 \%$ of the total number of the stem points and about $3 \%$ of the generated segments in plots 1 and 2 .

\subsubsection{Reference data}

The schematic class labels which groups individual segments into tree stems were later obtained based on the relative segment positions and orientations using visualization. In some cases a single tree stem was represented in the point cloud, but it was missing from the reference data, due to the lack of evidence in the $3 \mathrm{D}$ point clouds. The total number of the labeled stems were 196.

\subsubsection{Choice of parameters}

The various control parameter values that we used in our experiment for each level is summarized in the Tabel4.2. The values were assigned experimentally based on the forest characteristics. 


\begin{tabular}{|l|l|c|}
\hline Parameters & symbols & values \\
\hline Cylinder radius & $r_{\text {seg }}$ & $0.5 \mathrm{~m}$ \\
Cylinder length & $l_{\text {seg }}$ & $2.0 \mathrm{~m}$ \\
Angular deviation & $\theta_{t h r}$ & $30^{\circ}$ \\
Spatial distance weight & $w_{1}$ & 10 \\
Maximum spatial distance & $d_{C, \max }$ & $0.6 \mathrm{~m}$ \\
Maximum projected distance & $d_{p, \max }$ & $0.6 \mathrm{~m}$ \\
Maximum angular deviation & $d_{A, \max }$ & $20^{\circ}$ \\
Balance coefficient of energy terms & $\alpha$ & $0.1 \times n_{p}$ \\
\hline
\end{tabular}

Table 3.1: Control parameters for the single tree stem detection method; $n_{p}$ refers to the number of points inside the clusters.

\subsubsection{Evaluation}

In the current experiment we use the "recall" and "precision" measures to characterize the detection performance between detected and reference tree stems. The "recall" is defined as the ratio of the reference stem numbers which have at least one associated detected stems to the total number of reference stems. The "precision" expresses the count of detected stems that were successfully connected to reference stems as a fraction of the total number of detected tree stems. We considered the detected and reference stems as matched if the average projected distance between them was not more than $30 \mathrm{~cm}$. This value was derived based on the maximum DBH of trees in the target area.

\subsection{Results and Discussion}

The procedure for stem detection was applied to the both plots. The output of the single tree stem detection consists of a number of point sets which correspond to the individual stems. The method takes the advantage of the increased point density, which makes more laser reflections available underneath the canopy for regions of test plots dominated by conifers, due to the smaller footprint size compared to the standard ALS. In contrast, the deciduous tree stems are missing in the point clouds due to the dense canopy cover in leaf-on state, and no benefit was achieved despite the lower footprint size. In case that only sparse understory is below the tree base height, stem points are successfully detected by the expressed classifier training and stem line fitting method.

Fig. 3.6 shows the stem detection results for a sample plot (mixed with deciduous and coniferous trees) in three main levels of point, segment and object. The sample plot contains deciduous and coniferous trees. In Fig. 3.6a the classification results at the point level based on the high and low point probability on the tree stems are presented. The "positive" and "negative" groups of segments are classified using shape context, angular deviation from the world $Z$ and point probability statistics in the Fig. 3.6b. Finally, at the object level stems with the fitted ODR line (orthogonal distance regression 
with the $\ell_{1}$ norm) after merging are indicated in the Fig. 3.6c. In the current figure, we used the minimum stem point probability threshold of 0.6 to remove low probability points from the analysis.

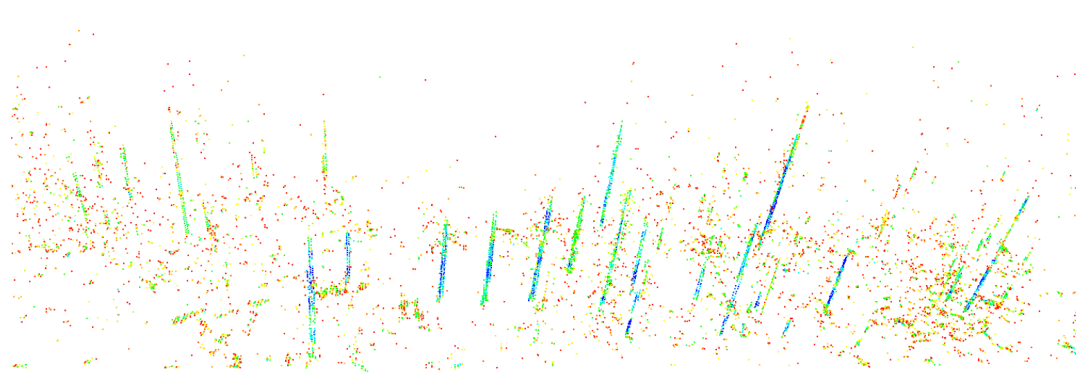

a)
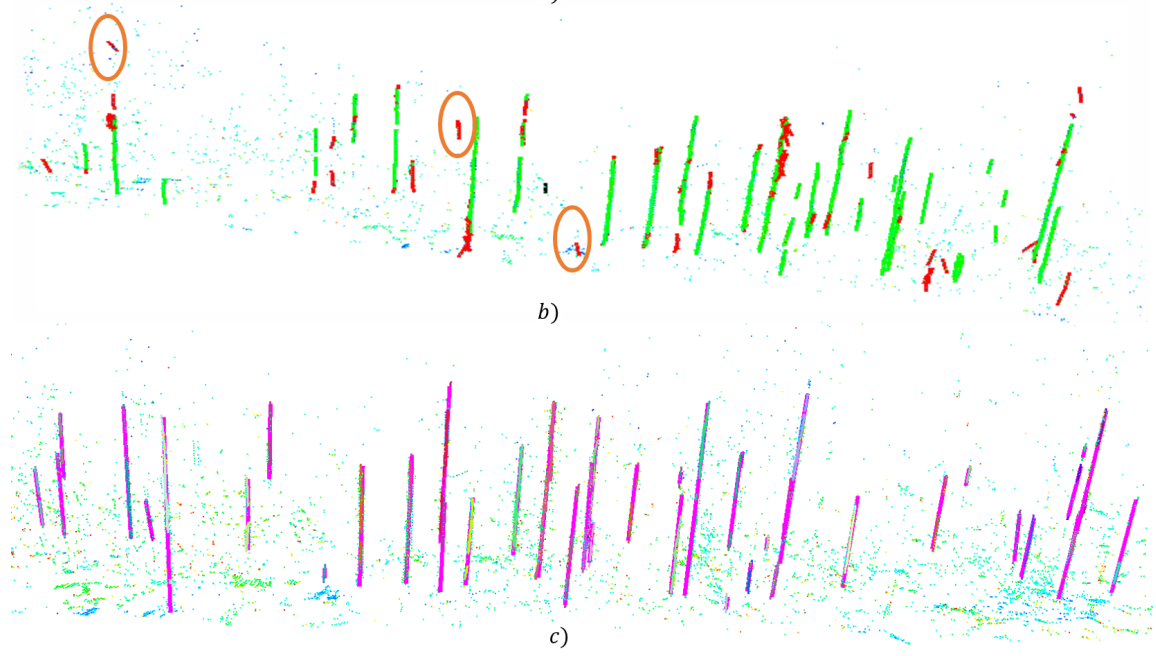

Figure 3.6: The detection results for a sample plot:(a), (b) and (c) correspond to point, segment and object levels, respectively (see Sections 3.2.1-3.2.3). At point level in (a), the red color shows low and blue high probability. The solid green bars in (b) indicate tree stems classified as positive and red bars refer to unmatched tree stems with references. The points which do not belong to the detected stems are removed from analysis and colored as cyan. Orange ellipses outline examples of the false alarms. The fitted magenta lines in (c) represent the reference tree stems which overlap with colored detected stems (ODR with the $\ell_{1}$ norm).

The detection performance of the proposed method is presented by Fig. 3.7. Note that in the current test plots, due to the point density and forest characteristics (particularly deciduous trees) up to $30 \%$ of the visible tree stems could not be detected. Specifically, in the lower canopy layer limited number of tree stems can be found since most of them are covered by taller tree stems and understory vegetation. Therefore, the majority of the detected stems are located in the upper and intermediate layer of the forest. 


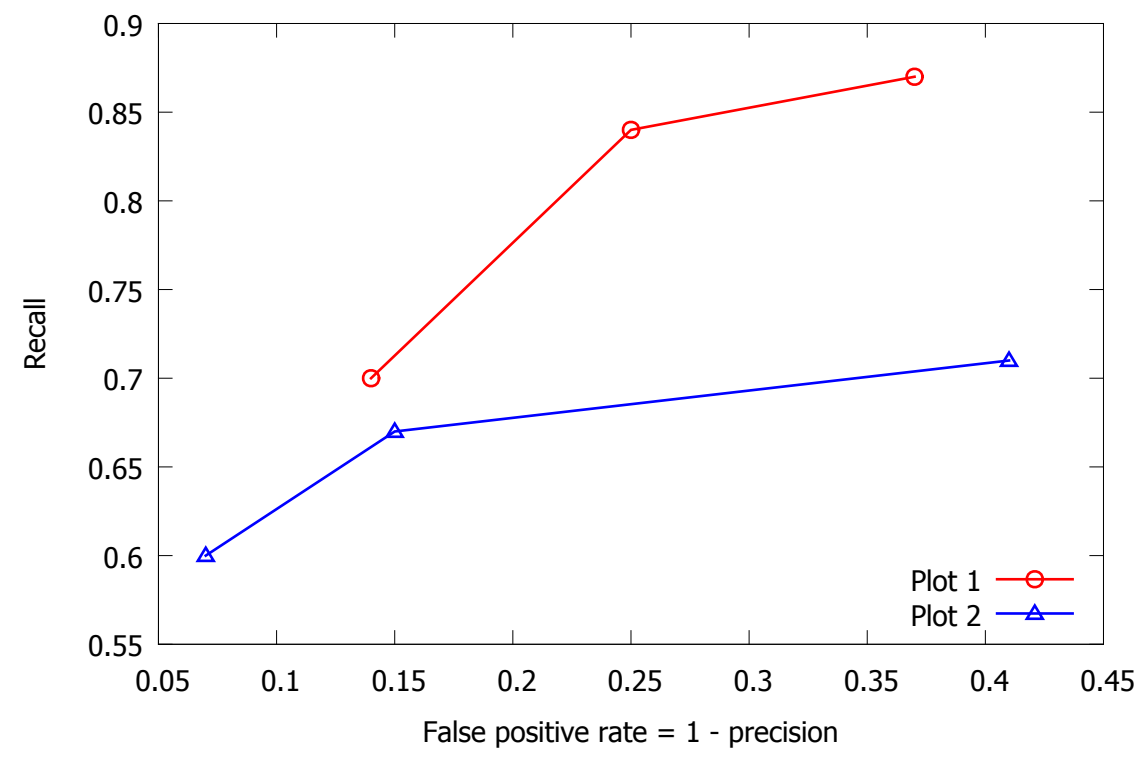

Figure 3.7: Single tree stem detection performance with probability threshold of 0.4 to 0.6 for the test plots.

Here, we focus on the detection accuracy of tree stems that are derived from the $3 \mathrm{D}$ point clouds. In the plot 1 , the good trade off results between recall and false positive rate are 0.84 and 0.25 , respectively. Also, for the plot 2 , the results are 0.63 for the recall and 0.13 for the false positive rate is achieved. By increasing the precision rate, the recall rate is decreased. The average rate of false detected tree stems in the plot 1 and 2 amount to 0.25 and 0.21 , respectively. However, no improvement is achieved in the lower layer and deciduous trees since (i) laser hits at the stem of small trees rarely happen, (ii) the stem points are missing for the trees with compact canopy. The restrictions of the approach are that only trees in visible stem in the $3 \mathrm{D}$ point cloud visualization can be detected. This problem could be alleviated by acquiring an even denser point cloud e.g. by using UAV-based laser scanning, where more stem hits are to be expected. As mentioned before, the method fails in the regions with high concentration of deciduous trees where stem hits are rare and stems points cannot be clearly clustered. In case of deciduous trees, it is not clear if an increase of the nominal point density of a ALS data with reducing flight height can provide more visible stems. Perhaps conducting the data acquisition in leaf-off state could remedy this problem. The current point density, did not allow to reconstruct the cylindrical shape of stems. Therefore the stem diameter estimation requires higher point density which is left for the future work. From the application point of view, the processing of large areas requires implementing a tiling scheme due to the memory usage of the spatial index structures as well as the large number of generated segment candidates. Moreover, the transferability 
of $3 \mathrm{D}$ descriptors is not perfect, in the sense that in our experiment we had to train and test our method on the same area. Another limitation is related to the optimization problem of the line fitting step. Due to the non-convexity, which does not guarantee global optimality, it might happen that the fitted line converge to local optimum.

\subsection{Conclusions}

The study presents a novel method for detecting stems of single trees based on the high density ALS data. Our results demonstrate that the classification precision is achieved to 0.86 and 0.85 , respectively for two sample plot 1 and 2 , with recall values of 0.7 and 0.67 . In future work, we would like to utilize the stems obtained from the proposed method to enhance the segmentation and delineation of single trees by providing prior knowledge about tree locations. Additionally, higher point density could lead to more precise reconstruction of the stems.

\section{Acknowledgment}

We would like to thank FMM GmbH Company for providing the laser scanning data. 


\section{Adaptive stopping criterion for top-down segmentation of ALS point clouds}

This chapter is based on:

Amiri, N., Polewski, M. P., Yao, W., Heurich, M., Krzystek, P., \& Skidmore, A. K. (2016). Adaptive stopping criterion for normalized cut segmentation of single trees in ALS point clouds of temperate coniferous forests. Poster presentation at 3rd workshop SIG on forestry, 15-16 September 2016, Krakow, Poland (pp. 1-1). Krakow: EARSeL.

Amiri, N., Polewski, P., Heurich, M., Krzystek, P., \& Skidmore, A. K. (2018). Adaptive stopping criterion for top-down segmentation of ALS point clouds in temperate coniferous forests. ISPRS Journal of Photogrammetry and Remote Sensing, 141, 265-274. 


\begin{abstract}
The development of new approaches on individual tree crown delineation for forest inventory and management is an important ongoing research issue. The increasing availability of high density ALS (Airborne Laser Scanning) point clouds offers the opportunity to segment the individual tree crowns and deduce their geometric properties with high accuracy. Top-down segmentation methods such as normalized cut are established approaches for delineation of single trees in ALS point clouds. However, overlapping crowns and branches of nearby trees frequently cause over- and under-segmentation due to the difficulty of defining a single criterion for stopping the partitioning process. In this work, we investigate an adaptive stopping criterion based on the visual appearance of trees within the point clouds. We focus on coniferous trees due to their well-defined crown shapes compared to deciduous trees. This approach is based on modeling the coniferous tree crowns with elliptic paraboloids to infer whether a given 3D scene contains exactly one or more than one tree. For each processed scene, candidate tree peaks are generated from local maxima found within the point cloud. Next, paraboloids are fitted at the peaks using a random sample consensus procedure and classified based on their geometric properties. The decision to stop or continue partitioning is determined by finding a set of non-overlapping paraboloids. Experiments were performed on three plots from the Bavarian Forest National Park located in Germany. Results show that, based on validation data from field inventory, our approach improves the segmentation quality by up to $10 \%$ across plots with different properties, such as average tree height and density. This indicates that the new adaptive stopping criterion for normalized cut segmentation is capable of delineating tree crowns more accurately compared to a static stopping criterion based on a constant NCut threshold value.
\end{abstract}




\subsection{Introduction}

Accurate measurements of forest resources are essential for precise and sustainable forest management (Chang et al., 2013). Single tree attributes such as tree crown base height, volume, DBH (Diameter at Breast Height), position, height and species are required for quantitative forest analysis and ecosystem services (Hu et al., 2014; Yao et al., 2012). Currently, most of those variables are estimated by measuring a set of sample plots manually in field surveys. Therefore, forest inventories are expensive and time consuming. During the last years, many studies have been focused on decreasing costs by developing inventory methods that are based on remote sensing techniques. Airborne Laser Scanning (ALS) has become a key tool for gathering information on 3D structures in forests (Wulder et al., 2012). The information derived from ALS data can provide detailed forest characteristics and serve as a basis for single tree analysis (Wagner et al., 2008; Reitberger et al., 2009). The information extracted from segmented trees, e.g. tree height or crown diameter, is often used in the role of independent variables in allometric modeling of additional individual tree characteristics such as stem volume, leaf area index and biomass as well as entire forest stands (Yao et al., 2012; Yu et al., 2011). Therefore, any inaccuracy of the tree delineation, which is often caused by over- or under-segmentation, will transfer to these characteristics. Various number of methods for detecting and delineating single tree crowns using ALS point clouds have been proposed in literature based on two main types of data: the ALS derived CHM (Canopy Height Model) and the original ALS point cloud. In the first type, tree crowns are found with the watershed algorithm (Pyysalo and Hyyppä, 2002) or with a slope-based segmentation (Hyyppä et al., 2001; Persson et al., 2002). The study of Persson et al. (2002) indicates a detection rate of $71 \%$ for a boreal forest dominated by spruce and pine trees. Later, Solberg et al. (2006) proposed a region growing method that starts from local surface maxima and finds crown polygons. The method was applied to a structurally heterogeneous spruce forest with an overall detection rate of $66 \%$ on the CHM, which was smoothed with a Gaussian filter. Heurich (2008) demonstrated that the segmentation method of Persson et al. (2002) leads to an average detection rate of $45 \%$ in the Bavarian Forest National Park. The segmentation results of the mentioned studies illustrate the strong dependency on the forest type.

On the other hand, point cloud based methods take advantage of the captured $3 \mathrm{D}$ information and focus on the detection of the single tree objects, which are either the tree as a whole or parts of the tree like stems and branches (Wu et al., 2016; Zhang et al., 2003). Several approaches have been developed for extracting single trees from ALS 3D point clouds. Morsdorf et al. (2004) used the $k$-means clustering algorithm to segment single trees from raw ALS point clouds. However, the accuracy of their study highly depends on seed points extracted from the CHM. Wang et al. (2008) subdivided the forest into different layers and applied a 2D morphological algorithm to obtain tree crowns. Reitberger et al. (2009) introduced a novel normalized cut segmentation method that extracts single trees using a graph cut approach. The study successfully showed that the overall accuracy of individual tree crowns 


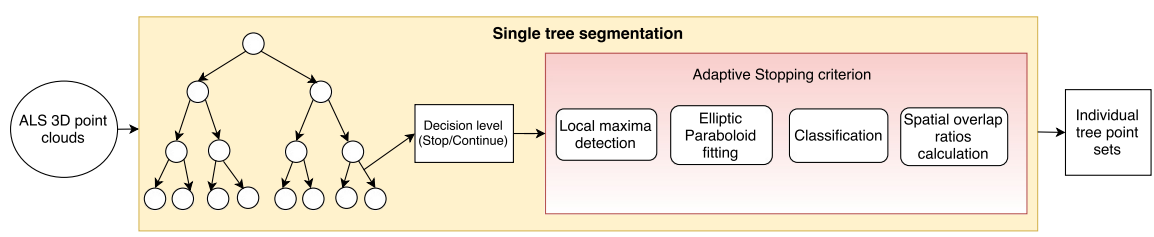

Figure 4.1: Overview of single tree segmentation strategy using adaptive stopping criterion.

in heterogeneous forest types could be significantly improved, especially in the lower forest layers (up to 20\%). Lee et al. (2010) proposed an adaptive region growing and clustering approach to detect single trees directly within raw point clouds. Li et al. (2012) developed a spacing-based algorithm which utilizes a region growing approach to segment trees in a coniferous mixed forest. Véga et al. (2014) suggested the PTrees method to extract trees in a forest from ALS data. The method is a multi-scale dynamic segmentation at point cloud level. Wu et al. (2016) developed an automated segmentation method which captures topological structure of forest and assesses topological relationships of tree crowns by using a graph theory-based localized contour tree method, achieving an overall accuracy of up to $94 \%$.

The forest structure has a strong impact on the single tree segmentation performance. Tree crowns have a complex shape that varies significantly from species to species. The accuracy of single trees delineation algorithms mainly depends on the forest complexity (Strîmbu and Strîmbu, 2015; GonzálezFerreiro et al., 2013; Vauhkonen et al., 2011). Moreover, the segmentation algorithms are controlled by many parameters, which are hard to estimate when the methods are applied to other forest types. This specification can be either explicit, as with the number of seed points in k-means, or implicit like the NCut threshold for spectral clustering methods. The incorrect setting of such parameters may lead to over- or under-segmentation effects in the delineated trees (Strîmbu and Strîmbu, 2015; Khosravipour et al., 2014; Yao et al., 2014; Li et al., 2012; Heurich, 2008). Although the control parameters can be estimated by a grid search method for a localized forest area, their transferability to larger scenes can be poor. Therefore, if the fixed scheme of control parameters in the segmentation algorithm is replaced by an adaptive scenario applied on the decision level, a more flexible tree crown delineation procedure is to be expected.

The main objective of this study is to develop a new adaptive stopping criterion, applicable to top-down segmentation methods for precise delineation of single trees in ALS 3D point clouds. Their parameters can be automatically trained from reference segmentations, alleviating the burden of manual, trialand-error parameter setting. Moreover, the adaptive procedure is based solely on the appearance of the target objects (tree crowns) within the point cloud, and is independent of any internal features of the underlying segmentation method. For the time being, we restrict our attention to coniferous trees whose well-defined crown shape can be easily modeled by an elliptic paraboloid 
(Koop, 1989; Husch et al., 2002). This study is motivated by the successful application of an adaptive stopping criterion for lying dead tree segmentation based on the normalized cut algorithm (Polewski et al., 2015b). Here, we extend the idea of an appearance-based adaptive stopping criterion to the domain of single tree segmentation. We conducted a series of experiments on sample plots from the Bavarian forest National Park to assess the performance of the proposed method using validation data from the field inventory. In our experiments, the normalized cut algorithm was used in the role of the segmentation method, but any other top-down clustering procedure could be applied instead.

The remainder of this work is structured as follows: Sections 2 and 3 describe the details of our approach; Section 4 illustrates the study area, materials, and field measurements. The results are presented and discussed in Section 5. Finally, the conclusions are stated in Section 6 .

\subsection{Top-down segmentation}

\subsubsection{Main foundation}

The segmentation of point clouds into individual objects in the scene is an initial step in processing 3D point clouds. The main objective of the segmentation processes is to divide points with similar attributes into homogeneous clusters. Among the various approaches, a popular paradigm is top-down segmentation, where initially all objects are assigned to a single cluster, which is then recursively partitioned. The subdivision continues until the predefined stopping criterion is met. However, the main difficulty of these methods is to define an appropriate stopping criterion which yields meaningful clusters under varying input scenarios.

\subsubsection{Normalized cut segmentation}

The normalized cut algorithm (Shi and Malik, 2000) is a top-down method for data segmentation. This method to construct a low-dimensional representation of the input 3D points uses the eigenvalues associated with the object similarity matrix (Polewski et al., 2015b). A graph is constructed based on the similarity matrix which quantifies pairwise compatibility between primitives from a predefined set, such as cubic voxels or irregular super-voxels provided by any kind of pre-segmentation like mean shift of $k$-means. A recursive bisection of the graph's vertices into disjoint clusters $A$ and $B$ is performed such that the within-cluster similarity is maximized while simultaneously the inter-cluster similarity is minimized. The corresponding normalized cut is:

$$
\operatorname{NCut}(A, B)=\frac{C u t(A, B)}{A \operatorname{ssoc}(A, V)}+\frac{C u t(A, B)}{A \operatorname{ssoc}(B, V)}
$$

with $\operatorname{Cut}(A, B)=\sum_{i \in A, j \in B} w_{i j}$ as the total sum of the weights between the segment $A$ and $B$, while $A \operatorname{ssoc}(A, V)=\sum_{i \in A, j \in V} w_{i j}$ is the sum of weights of all edges ending in segment $A$. The similarity function for normalized cut 
is based on the pair-wise similarity of the clusters. The mentioned segmentation is controlled by several parameters whose values can be optimized in experiments. The most important parameter that controls the subdivision of the graph is the normalized cut threshold $N C u t_{t h r}$, which has no physical interpretation. During the segmentation, when the NCut value of the obtained clusters A and B exceeds $N C u t_{t h r}$ the similarity between A and B is too high and the process must be terminated. Clearly, choosing a suitable threshold is critical to obtaining a reasonable segmentation, because a too small NCut thr will lead to under-segmentation with clusters consisting of unrelated objects. On the other hand, a too big value will result in oversegmentation and many small clusters. In real world applications, setting the most suitable value for this static threshold is challenging due to different input characteristics.

\subsubsection{Other top-down algorithms}

Although normalized cut is the most prominent representative of the topdown segmentation algorithms, other methods have also found use in various clustering applications. Two methods from the graph-cut family are Min Cuts (Wu and Leahy, 1993) as well as MinMax Cut (Nie et al., 2010), which partition the graph according to different optimization objectives. Also, a recursive, bisecting version of K-means has been developed (Savaresi and Boley, 2001). Essentially, any of these mentioned algorithms could benefit from the proposed adaptive stopping criterion approach. In this work we decided to utilize the normalized cut procedure as the core method, because (i) the implementation could potentially be modified to optimize one of the other related criteria from the spectral clustering family (Min Cuts, MinMax Cut) with moderate effort, and (ii) the normalized cut has already been applied in literature for the studied problem of tree segmentation.

\subsection{Adaptive stopping criterion}

\subsubsection{Outline}

Consider a point cloud representing a forest scene with multiple coniferous trees. The normalized cut algorithm recursively partitions the $3 \mathrm{D}$ data, starting with the entire point cloud, until the level of single trees is reached. Let $S_{i}$ represent an intermediate point cluster obtained at recursion level $m$ of the partitioning. $S_{i}$ may contain one or more trees. The main idea behind our adaptive stopping criterion is to detect tree crowns by fitting local quadric surfaces to candidate tree tops, and using this information to determine whether the currently processed cluster of points represents a single or multiple trees. In the former case, the segmentation is stopped, otherwise the current cluster is split and the partitioning process continues. The method proceeds as follows. We use a local maxima detection approach to find candidate peaks of single trees. Then, we apply the RANdom SAmple Consensus (RANSAC) method to estimate the best fitting quadratic surface parameters for points 
around each detected local maximum. The signed distances between the fitted surface and local points are binned to form histogram features. These features provide a basis for classifying the neighborhood of each local maximum either as a true tree top or a false positive. After classification in a probabilistic manner, the spatial overlap ratios $o p_{r}$ (proportion of shared volumes) between all pairs of positively classified candidate tree tops are calculated. If a pair of fitted surfaces have an overlap ratio below a threshold value $\max _{o p_{r}}$, then it is decided that the current cluster contains more than one tree and the segmentation has to proceed. If no such pair is found, the stopping criterion is activated and the segmentation of the current cluster is terminated. The entire processing pipeline is presented in Fig.4.1. In the following, we explain the steps of our method in detail.

\subsubsection{Local maxima detection}

The input ALS data is a set of $3 \mathrm{D}$ point clouds with $3 \mathrm{D}$ coordinates $p_{i}\left(x_{i}, y_{i}, z_{i}\right)$ for each point. In our approach, the local maxima are detected only within a currently segmented scene in the normalized cut segmentation process. We examine a spherical neighborhood around each point to determine whether is has the locally maximal $z$ coordinate or not. The neighborhood radius is balanced between allowing nearby tree tops and not producing too many insignificant local maxima. Note that, we do not apply smoothing step to the original point clouds. Fig.4.2 shows the detected local maxima for two clusters $S_{i}$ and $S_{j}$ with true tree tops and a false positive, respectively.
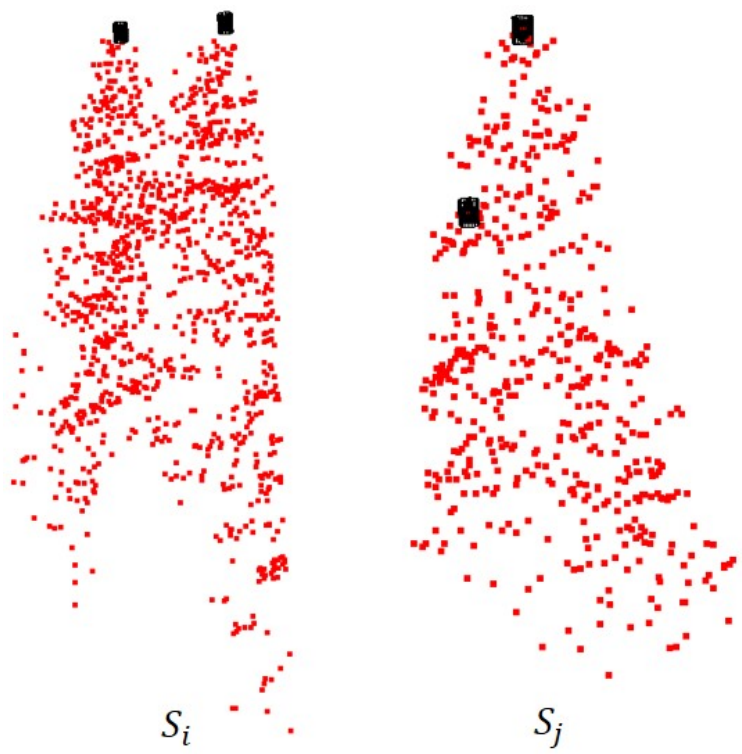

Figure 4.2: Detected local maxima for two pair of clusters $S_{i} ; S_{j}$. The $S_{i}$ represents a cluster with 2 true tree tops; the $S_{j}$ shows a false positive scenario in the cluster with at least one false detected local maximum. 


\subsubsection{Shape fitting with RANSAC}

The RANdom SAmple Consensus algorithm (Fischler and Bolles, 1981) is a general robust parameter estimation approach designed to deal with a large proportion of outliers in the input data. In this step, the mentioned algorithm is applied to estimate the best fitting elliptic paraboloid parameters around each local maximum based on points inside a cylinder with a predefined length $c y l_{l}$ and radius $c y l_{r}$. The mentioned parameters of the cylinder are defined experimentally. The center of the paraboloid is indicated by the detected local maximum, and RANSAC is used to compute the remaining paraboloid parameters. Fig.4.3 illustrates the fitted elliptic paraboloids based on detected local maxima for two clusters $S_{i}$ and $S_{j}$.

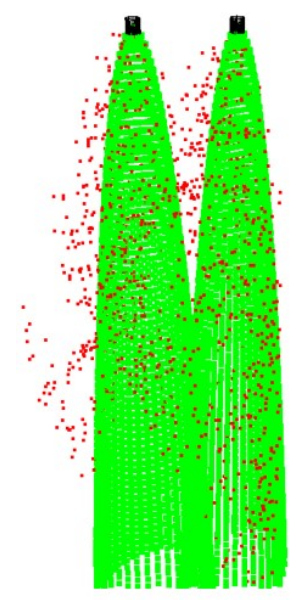

$S_{i}$

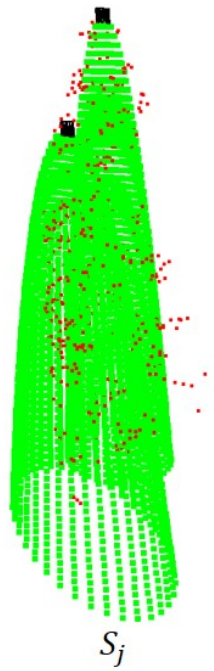

$S_{j}$

Figure 4.3: Fitted paraboloid surfaces on detected local maxima. The $S_{i}$ represents a cluster with two true tree tops; the $S_{j}$ shows a false positive cluster with at least one wrong detected local maximum.

\subsubsection{Elliptic paraboloids}

A second order algebraic surface is given by the following general equation

$$
\begin{aligned}
& a x^{2}+b y^{2}+c z^{2}+2 f y z+2 g z x+2 h x y+2 p x+2 q y+2 r z+d=0 \\
& E=\left[\begin{array}{llll}
a & h & g & p \\
h & b & f & q \\
g & f & c & r \\
p & q & r & d
\end{array}\right]
\end{aligned}
$$


where $E$ stands for the coefficient matrix of the surface. The quadratic surfaces have different standard form types. In this study, we use the elliptic paraboloid, a quadratic surface which has an elliptical cross section (Dai et al., 2007). We assume the paraboloid axis is known and coincides with the world $Z$ axis due to the phenomenon of gravitropism of trees. Therefore, the simplified version of the Eq.4.2 as an elliptic paraboloid of height $Z$, semi-major axis $a$ and semi-minor axis $b$ without any rotation angle can be specified parametrically as a function of $(x, y)$ :

$$
Z(x, y)=-\frac{\left(x-x_{c}\right)^{2}}{a^{2}}-\frac{\left(y-y_{c}\right)^{2}}{b^{2}}+z_{c}
$$

The paraboloid's center $\left(x_{c}, y_{c}, z_{c}\right)$ is fixed to the current detected local maximum, whereas the semi-axis lengths $a$ and $b$ need to be determined through RANSAC estimation.

\subsubsection{Details of RANSAC estimation}

Consider two samples $\left(x_{0}, y_{0}, z_{0}\right)$ and $\left(x_{1}, y_{1} ; z_{1}\right)$ with $\left(x_{c}, y_{c}, z_{c}\right)$ as the fixed center of paraboloid. For the mentioned samples, by using the following equation (Eq.4.5) it is possible to calculate the axis lengths $a$ and $b$.

$$
\left[\begin{array}{ll}
\left(x_{0}-x_{c}\right)^{2} & \left(y_{0}-y_{c}\right)^{2} \\
\left(x_{1}-x_{c}\right)^{2} & \left(y_{1}-y_{c}\right)^{2}
\end{array}\right]\left[\begin{array}{l}
1 / a^{2} \\
1 / b^{2}
\end{array}\right]=\left[\begin{array}{l}
z_{0}-z_{c} \\
z_{1}-z_{c}
\end{array}\right]
$$

After calculating the axis lengths $a$ and $b$, for all points their distances to the paraboloid surface are determined. Additionally, the absolute distance $\left|z-z_{i}\right|$ is taken as the error measure for the RANSAC.

\subsubsection{Classification}

Although the RANSAC procedure yields the optimal paraboloid shape anchored at the chosen local maximum, it is still possible that the paraboloid does not represent a true, distinct tree top, but rather is located at the side of an adjacent, dominant tree (see Fig.3). Therefore, it is necessary to further classify each fitted paraboloid based on its spatial characteristics in order to retain only the ones representing true tree tops. We use the kernelized logistic regression (KLR) with $L_{2}$ norm regularization as classifier. Logistic regression models the probability distribution of the class label $Y$ and histogram features $X$ (see section 3.4.1) as follows:

$$
P(Y=1 \mid X=x)=\frac{1}{1+\exp \left[-\sum_{j=1}^{N} \alpha_{j} k\left(x_{i}, X\right)\right]}
$$

where $j, j=1 \ldots N$ denotes $N$ feature vectors of training examples and $Y$ the corresponding binary label. The term $k$ represents a positive semi-definite kernel function.

Training the model amounts to maximizing the regularized log-likelihood of the training examples in the Eq.6 as:

$$
\max _{\alpha} \quad \ell(\alpha)-\frac{\lambda}{2} \alpha^{T} K \alpha
$$


where $K=\left(k\left(x_{i}, x_{j}\right)\right)_{1 . . N, 1 . . N}$ represents the design matrix, and the expression $\alpha^{T} K \alpha$ denotes the $L_{2}$ norm regularization term. Eq.7 represents a convex optimization problem and we solved it by the Newton-Raphson method (Roscher et al., 2012). The model's log-likelihood and the functional form of the used Gaussian kernel are given respectively by Eqs.8 and 9 .

$$
\begin{gathered}
\ell(\alpha)=\sum_{j=1}^{N} \log P\left(Y=y_{i} \mid X=x_{i}\right) \\
K_{\gamma}\left(x_{i}, y_{j}\right)=\exp \left(-\frac{1}{2} \frac{\left|x_{i}-x_{j}\right|^{2}}{\gamma}\right)
\end{gathered}
$$

where $x_{i}$ and $x_{j}$ are two sample feature vectors. The two main parameters, Gaussian kernel bandwidth $\gamma$ and regularization coefficient $\lambda$, are determined through grid search on an exponential grid, using Cohen's kappa coefficient as the error measure and 10-fold cross-validation.

We classify the candidate local maxima (and their associated paraboloids) of single coniferous trees into two classes of "positive" and "negative", respectively. The "positive" class corresponds to true tree tops, whereas the "negative" class indicates false positives. See Sec. 4.2 for a description of the training procedure.

\subsubsection{Elliptic paraboloids features}

The features for classifying the local maxima are based on projected distances of local points to the fitted shape. Specifically, we consider all points located in the aforementioned cylinder around the local maximum (see Sec. 3.3) and compute an approximate projection onto the paraboloid using algebraic distance, i.e. for a point $Q_{i}=\left(x_{i}, y_{i}, z_{i}\right)$ we take the point $\left(x_{i}, y_{i}, Z\left(x_{i}, y_{i}\right)\right)$, where $Z\left(x_{i}, y_{i}\right)$ is the fitted surface's $Z$ position at coordinate $(x, y)$, as in Eq. 4. We decided to use this approximate method instead of true projection onto the paraboloid due to the fact that computing the true projection of a point onto a quadric requires solving a 6 -th degree polynomial equation for each point (Dai et al., 2007), which could be prohibitively computationally expensive. The signed distance from point $Q_{i}$ to the surface is thus $z_{i}-$ $Z\left(x_{i}, y_{i}\right)$. In this part of our method, the signed distances are binned to form histogram features $X$ for the classification purpose mentioned in the section 3.4. These features capture the shape of point distribution around the paraboloids apex. Fig.4.4 shows the process of generating features for the local maxima classification. For local maxima representing true tree tops, the distances should be approximately symmetrically distributed around zero, while for the case of a false local maximum depicted in Fig.4.3 $\left(S_{j}\right)$, the signed distance distribution should be significantly biased towards large positive residuals.

\subsubsection{Calculating spatial overlap ratio}

After the classification step, a number of fitted paraboloids remain which represent the detected true tree tops. However, in some cases more than one 

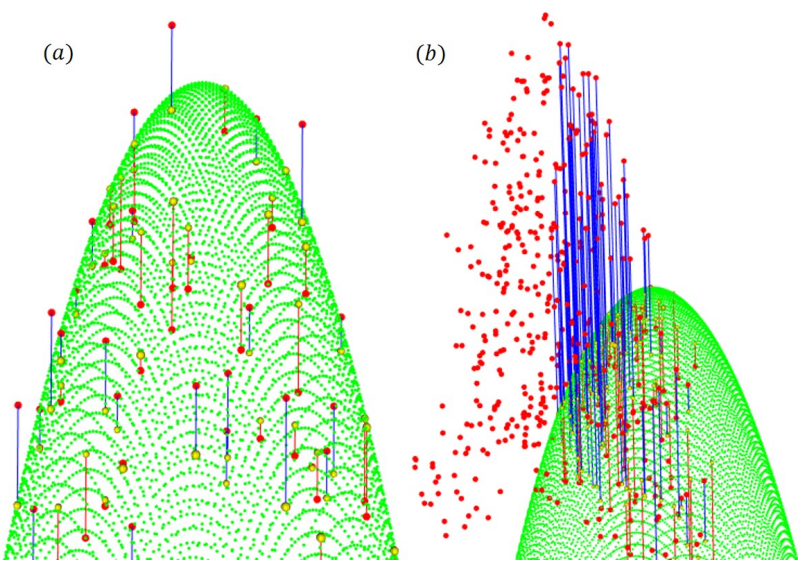

Figure 4.4: Signed distances between data points (in red) and their approximate projections (in yellow) onto the fitted paraboloid (green). Positive and negative distances are indicated respectively by blue and red lines. (a) Residuals around a true tree top, distributed symmetrically around zero, (b) Residuals around false local maximum located at the side of the tree, showing bias towards large positive values.

local maximum may represent the same tree, which makes it necessary to filter them out. Therefore, we define a feature for a pair of paraboloids $S_{i}$ and $S_{j}$ which evaluates the ratio of their spatial overlap $o p_{r}$, i.e. the ratio of volume shared by both shapes to the volume of an individual paraboloid. The ratio is normalized between a value of one and zero, indicating no overlap between the paraboloids, and one, corresponding to full paraboloid overlap. If the overlap ratio exceeds the maximum value $\max _{o p_{r}}$, then we assume that both paraboloids represent the same tree. In the current step, since it is difficult to analytically derive parameters for elliptic surfaces, we apply the Monte Carlo simulation method to estimate the spatial overlap of the fitted paraboloids. Fig.4.5 represents the idea behind calculating the intersection volume using a random simulation method. We generate a number of sample points $N$ within the interior of the first paraboloid. Afterwards, for each point we check the possibility if that point lies also in the second paraboloid. The overlap ratio $o p_{r}$ can be approximated as the number of points which are located inside both paraboloids divided by the number of generated sample points $N$. Note that in the current experiment, a uniform spatial distribution of the points is generated in the entire volume of the paraboloid. To ensure this uniformity, we perform the sampling with two steps. In the first step, we draw the vertical distance $Z$ from the paraboloid center randomly according to the triangular distribution, $P(Z \leq z) \propto z^{2}$. For the second step, we generate a point from the interior of the ellipse which constitutes the cross section of the paraboloid at the drawn height of $z$ from the previous step. Fig.4.6 shows the process of uniformly sampling points in the paraboloid. In order to maintain a uniform point density across the entire paraboloid, the local densities in every vertical 'slice' of the volume should be equal. However, the 

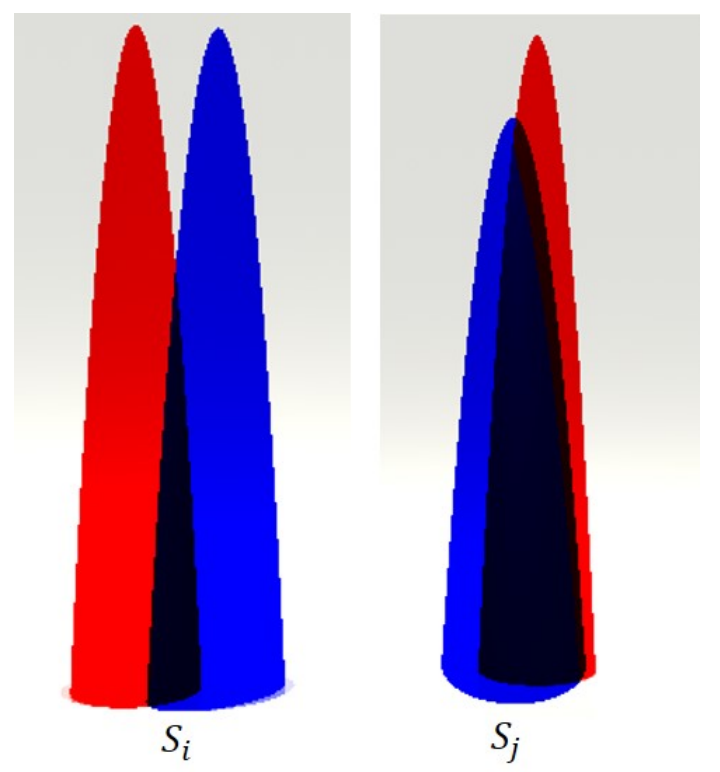

Figure 4.5: Estimating the spatial overlap ratio $o p_{r}$ between fitted paraboloid surfaces. The $S_{i}$ represents a cluster with 2 true tree tops; the $S_{j}$ shows a false positive cluster with at least one false detected local maximum.

volume of a slice at height $z$ is proportional to $z$. Since the density remains constant, the number of generated points at a layer must also be proportional to its height. The spatial overlap ratio of paraboloids $o p_{r}$ for the adaptive stopping criterion method is given by algorithm Alg.1. The rnd() function in the algorithm refers to the uniform random number generator in the range 0 to 1 . This Monte Carlo based procedure yields an unbiased estimator of the true ratio of overlapping volume.

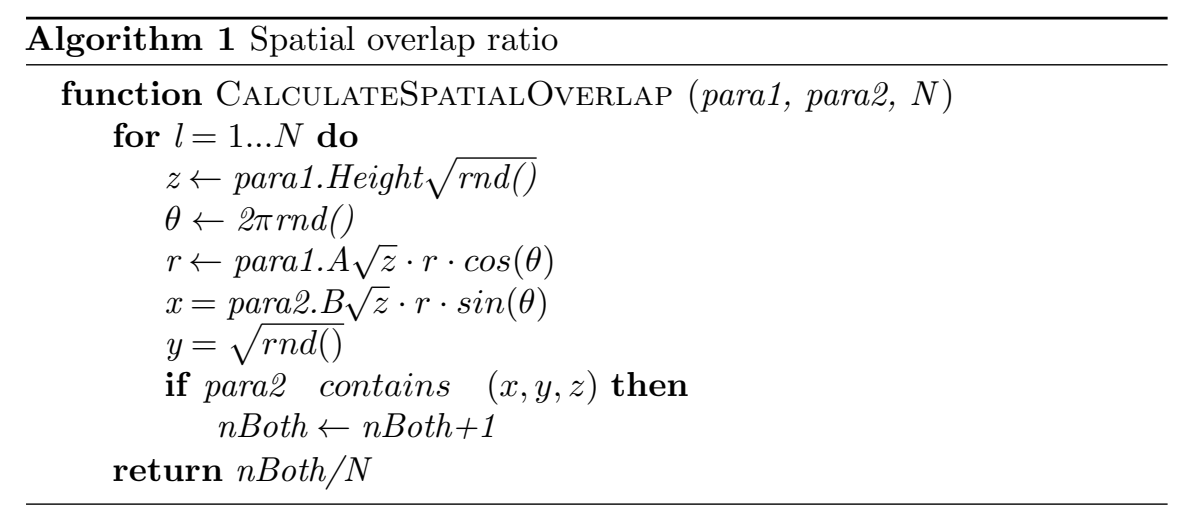




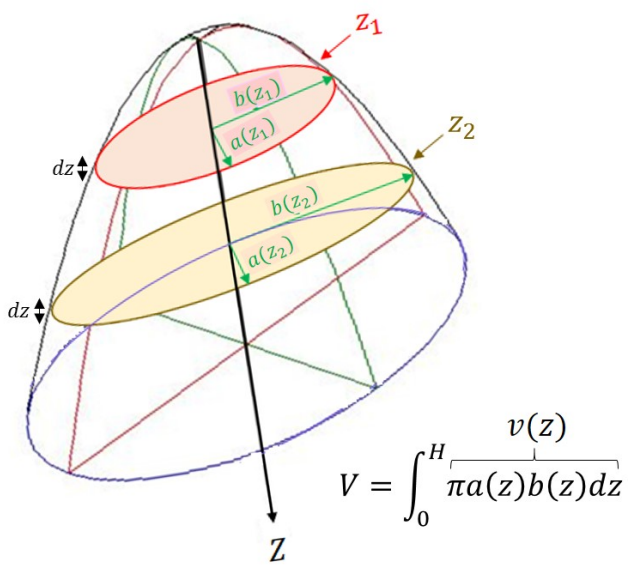

Figure 4.6: The entire volume of a paraboloid $V$ can be decomposed into an infinite number of elliptical slices with infinitely small thickness $d z$ and volumes $v(z)$ which are functions of their heights $z$. $H$ represents the height of the paraboloid. The number of points generated in each slice should be proportional to its cross section area.

\subsection{Experiment}

\subsubsection{Materials}

Our experiments were conducted for three sample plots in the Bavarian Forest National Park $\left(49^{\circ} 3^{\prime} 19^{\prime \prime} \mathrm{N}, 13^{\circ} 12^{\prime} 9^{\prime \prime} \mathrm{E}\right)$, a temperate forest located in the southeastern part of Germany along the border to the Czech Republic. The sample plots contain a mixture of mountainous and subalpine forest types dominated by Norway spruce (Picea abies) and European beech (Fagus sylvatica) (Cailleret et al., 2014). The airborne full waveform data were acquired using a Riegl LMS-Q560 scanner in May 2007 in a leaf-on condition with an average point density of 25 points $/ \mathrm{m}^{2}$. The flying altitude of 400 $\mathrm{m}$ resulted in a footprint size of $20 \mathrm{~cm}$. We used the mixture-of-Gaussians decomposition model (Reitberger et al., 2009) on the collected waveforms, obtaining a 3D point cloud. The 3D visualization of point clouds for the plots (coniferous-dominated stands) is shown by Fig.4.7. Table 1 summarizes the characteristics of our sample plots, estimated based on the reference data from field inventory.

\subsubsection{Classifier training}

Additionally, we chose 100 point cloud clusters from areas outside of the test plots as training data for the local maxima classifier. The clusters consisted both of scenes containing only a single tree and ones comprising multiple adjacent trees, in order to ensure a possibly wide range of training scenarios 

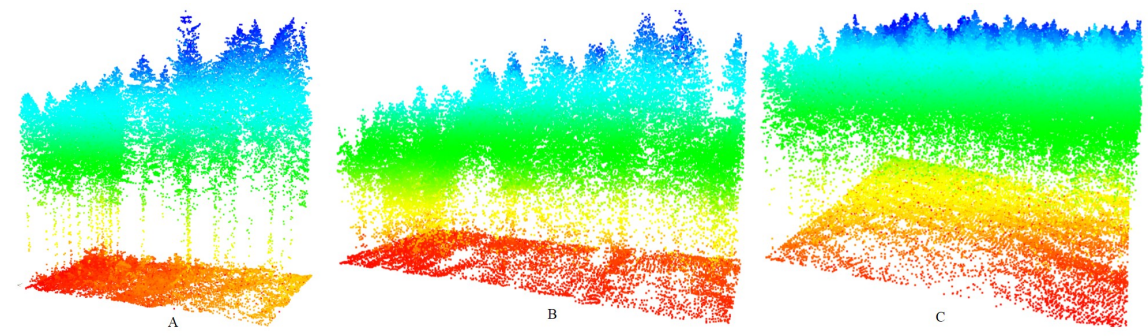

Figure 4.7: ALS point clouds of Plot A (100\% coniferous), Plot B (99\% coniferous) and Plot $\mathrm{C}$ (70\% coniferous) colored by height over DTM.

Table 4.1: Properties of sample plots.

\begin{tabular}{cccc}
\hline Property & Plot A & Plot B & Plot C \\
\hline Size [ha] & 0.10 & 0.10 & 0.30 \\
Trees/ha & 450 & 2150 & 700 \\
Ave. DBH* (cm) & 46.2 & 17.9 & 35 \\
Ave. Crown base height $(\mathrm{m})$ & 20.9 & 7.08 & 16.7 \\
Ave. Tree height (m) & 36.9 & 16.08 & 35.45 \\
Dominant species & Spruce & Spruce & Spruce \\
Deciduous trees[\%] & 0 & 1 & 29 \\
Understory trees [Number] & 0 & 76 & 11 \\
Intermediate layer [Number] & 4 & 85 & 33 \\
Overstory trees [Number] & 41 & 54 & 165 \\
\hline
\end{tabular}

*Diameter at Breast Height.

(with the proportion of 50\%-50\%). These clusters were obtained from various intermediate steps, i.e. partial segmentations, of the standard normalized cut algorithm with the static normalized cut threshold $N C u t_{t h r}$. For each training cluster, the local maxima detection was performed, and each local maximum was labeled as either a true tree top or a false positive based on visual interpretation of the point cloud. The paraboloid-based features (see section 3.4.1) were extracted for each local maximum. The set of all extracted features together with the local maxima labels formed the basis for training the classifier to detect the characteristic paraboloid shape of coniferous trees. The classifier provides a probability of $p$ that a local maximum represents a 'true positive', i.e. tree top. Later, the minimum acceptance probability threshold min $_{p_{t h r}}$ is used as a control parameter, i.e. a local maximum is processed further is its probability $p$ exceeds this threshold value.

\subsubsection{Reference data}

The ground truth data for the test plots was acquired by field measurements. In each of the 3 plots, at least 40 single trees with diameter at breast height $(\mathrm{DBH})$ bigger than $10 \mathrm{~cm}$ were present. Several individual tree parameters such as total tree height, stem position, DBH and tree species were 
measured with the help of GPS and tacheometry. Moreover, the single trees in the scene are subdivided into three layers with respect to the top tree height $h_{t o p}$ in the plot. The top tree height $h_{t o p}$ is defined as the average height of the 100 highest trees per ha (Heurich, 2006). The lower layer contains all trees below $50 \%$ of $h_{t o p}$, the intermediate layer corresponds to all trees between $50 \%$ and $80 \%$ of $h_{t o p}$, and the upper layer refers the rest of the trees. Plot A has no trees at the understory layer and less than $10 \%$ of total number of trees at the intermediate layer. However, plot B contains higher number of trees at intermediate and understory layer compared to plots $\mathrm{A}$ and $\mathrm{C}$. Plot $\mathrm{B}$ has the highest number of trees at the intermediate and understory layers among the other plots. In this study, we removed both lower and intermediate layers based on the top tree height $h_{t o p}$ and focused on the upper layer, where the single tree crowns are clearly shaped as elliptic paraboloids. The total number of single trees in the three sample plots was 260.

\subsubsection{Experimental setup}

We conducted 4 groups of experiments. These experiments are concerned with assessing the performance of the entire method for the single tree segmentation particularly the benefit of using the newly introduced adaptive stopping criterion. For the first two sets, we used the basic normalized cut segmentation algorithm with normalized cut threshold $N C u t_{t h r}$. In the other two groups of experiments, the tree segmentation was based on our adaptive stopping criterion. To demonstrate that our approach is independent of the segmentation granularity, we executed the experiments with two kinds of primitives for merging: (i) voxel-based and (ii) obtained from mean shift clustering. In the voxel-based approach, the main idea is to subdivide the tree into a voxel space, which results in equal-sized primitives in the form of voxels with a side length $d_{v o x}=0.5 \mathrm{~m}$. On the other hand, the mean shift algorithm generates a segmentation of the point cloud consisting of non-uniformly sized clusters. We used a cylindrical kernel with base radius $h_{r}=2.4 \mathrm{~m}$ and height $H=2.4 m$ (See Yao et al. (2013)). The similarity function was the standard exponential model as in Reitberger et al. (2009). The graph's adjacency relation was based on a cylindrical neighborhood with a predefined radius and unlimited height, as described in our previous work (Amiri et al., 2016). Aside from geometric information, the similarity function contained a term reflecting the mean pulse intensities and widths averaged over the clusters' member points.

\subsubsection{Choice of parameters}

The different control parameter values that we used in our approach are summarized in the Table 4.2. The values were assigned empirically based on the forest characteristics. The cylinder radius $c y l_{r}$ is based on the largest radius of a single coniferous tree crown which we expect to find in the plots. Similarly, the cylinder length $c y l_{l}$ approximates the maximum expected range of the upper tree crown in the study area. Moreover, the local maxima 
neighborhood radius $r_{n e i g}$ corresponds roughly to the average size of the tree crown segments. All parameter values were reused for all test plots.

Table 4.2: Control parameters of the adaptive stopping criterion method for single tree segmentation.

\begin{tabular}{llc}
\hline Parameters & symbols & values \\
\hline Maximum overlap ratio & max $_{o p_{r}}$ & 0.3 \\
Minimum probability threshold & min $_{p_{t h r}}$ & 0.5 \\
Local maxima neighborhood radius & $r_{n e i g}$ & 1.2 \\
Signed distance bin width & $s d_{b w}$ & 1.0 \\
Signed distance range & $s d_{r a n g e}$ & 20.0 \\
Cylinder length & $c y l_{l}$ & 5.0 \\
Cylinder radius & $c y l_{r}$ & 1.0 \\
RANSAC inlier distance & sac $_{d}$ & 0.05 \\
\hline
\end{tabular}

\subsubsection{Evaluation}

The output of our processing pipeline consists of set of points which correspond to the individual segmented trees. The matching between segmented and reference trees was calculated using the strategy proposed by Reitberger et al. (2009). We considered the segmented and reference trees as matched if (i) the distance to the reference single tree is smaller than $60 \%$ of the mean tree distance within the sample plot and (ii) the height difference between and the height of the reference tree is smaller than $20 \%$ of the top height of the plot. Moreover, if a reference tree is associated with more than one tree position, the tree position with the shortest distance to the reference tree is taken. A segmented tree cluster without a link to a reference tree is called a false positive segment.

In the current experiment, we use the 'correctness' and 'completeness' metrics to measure the quality of the obtained segmentation results. The 'correctness' metric is defined as the number of segmented trees that were successfully linked to reference trees as a fraction of the total number of segmented trees. The 'completeness' expresses the ratio of the number of reference trees which have at least one associated segmented tree to the total number of reference trees.

\subsection{Results and discussion}

\subsubsection{Sensitivity analysis}

Clearly, the adaptive stopping criterion approach requires proper values for the control parameters. We conducted tests to find the most important parameters' values and their sensitivity. We demonstrate the performance of the adaptive stopping criterion with two main control parameters: the maximum overlap ratio $\max _{o p_{r}}$ and minimum probability threshold $\min _{p_{t h r}}$. The results from the maximum overlap ratio $\max _{o p_{r}}$ showed that the smaller values 
produce relatively higher correctness and completeness, respectively. A value of 0.3 represents a good trade off between the correctness and completeness which can successfully split the cluster into adjacent tree crowns. Fig.4.8 shows the segmentation performance by ROC curves on the sample plots mentioned in the section 4.2 when different values of maximum overlap ratio $\max _{\text {op }_{r}}$ are applied. The three plots dominated by coniferous trees share the properties that at the threshold of 0.3 of the spatial overlap between clusters, over $70 \%$ of trees can be detected correctly. In plot C, which contains almost $30 \%$ deciduous trees, the true positive rate on average did not exceed $65 \%$ for various threshold values. Among the test datasets, plot A exhibits the relatively higher correctness and completeness rates.

In Fig. 4.9 the adaptive segmentation performance for the probability threshold of the same sample plots (see section 4.2) is presented by ROC curves. For the minimum probability threshold $\min _{p_{t h r}}, 0.5$ was selected as an optimal trade off value. A larger minimum probability threshold $\min _{p_{t h r}}$ led to more segments, which resulted in an overall higher completeness but lower correctness across the entire sample plots. The sensitivity analysis of our segmentation method for the selected plots was similar if a minimum probability threshold $\min _{p_{t h r}}$ value smaller than 0.3 was selected. Also, on the plot A the ROC curves with different threshold values continue upwards and attain a completeness of 0.75 , whereas on the other two plots, a value of 0.65 is not exceeded. The lowest true positive rate with the highest false positive rate is achieved by plot B. Note that the obtained values of 0.3 for the maximum overlap ratio $\max _{o p_{r}}$ and 0.5 for the minimum probability threshold min $_{p_{t h r}}$ were nearly optimal on all three considered plots.

Moreover, for the baseline experiment, we demonstrate the sensitivity analysis for the normalized cut threshold $N C u t_{t h r}$ which controls the subdivision of the segments in the procedure. Tests in terms of correctness and completeness for the sample plots mentioned in the section 4.2 showed the best performance was achieved by the value of 0.16 .

Table 4.3: Results of analysis on the upper canopy layer for the sample plots $\mathrm{A}, \mathrm{B}$ and $\mathrm{C}$.

\begin{tabular}{cccc}
\hline Segmentation scenario & Plot A & Plot B & Plot C \\
\hline \hline & Completeness & & \\
\hline Mean Shift + NCut & 0.69 & 0.56 & 0.61 \\
Voxel-based + NCut & 0.66 & 0.58 & 0.61 \\
Mean Shift + NCut (Adaptive) & 0.76 & 0.67 & 0.68 \\
Voxel-based + NCut (Adaptive) & 0.77 & 0.67 & 0.69 \\
\hline \hline & Correctness & & \\
\hline Mean shift + NCut & 0.59 & 0.64 & 0.70 \\
Voxel-based + NCut & 0.53 & 0.63 & 0.69 \\
Mean shift + NCut (Adaptive) & 0.68 & 0.70 & 0.79 \\
Voxel-based + NCut (Adaptive) & 0.65 & 0.70 & 0.76 \\
\hline \hline
\end{tabular}




\subsubsection{Adaptive segmentation approach}

The results of the segmentation performance on the upper canopy layer with respect to the two set of merging primitives: (i) voxel-based and (ii) obtained from mean shift clustering are summarized in the Table 4.3. For all plots, the correctness and completeness terms are estimated. In the current plots, due to the point density and forest characteristics (particularly deciduous trees) up to $30 \%$ of the upper layer trees could not be correctly segmented. The task of adaptive single tree segmentation in the mixed deciduous and coniferous plots proved to be even more challenging than it was for the coniferous dominated stands. In upper canopy layer, the reliability of the method is high because the 3D tree structure captured within the point clouds does exhibit the full shape of elliptic paraboloids.

The results in terms of correctness and completeness for the adaptive segmentation by using two different primitives are presented. For three plots, an improvement of $6-9 \%$ and $7-10 \%$ respectively for correctness and completeness is achieved, compared to the basic normalized cut segmentation with mean shift primitives. Also, the adaptive approach of the normalized cut with voxel-based primitives for the same plots compared to the basic segmentation with voxel-based step performs a gain of $7-8 \%$ and $8-10 \%$ respectively in terms of correctness and completeness. The experiments on three plots confirm that the higher completeness and correctness rate can be achieved for the adaptive normalized cut with voxel-based and mean shift clustering primitives compared to the basic segmentation approaches. In the case of adaptive segmentation by voxel-based primitives, for plot A the good trade off results between correctness and completeness rate are 0.65 and 0.77 , respectively. For plot B, identical correctness and completeness results for both voxel-based and mean shift primitives are achieved. Finally, for plot C, 0.69 for the completeness and 0.76 for the correctness rate are accomplished. For all the plots, when the adaptive segmentation scenario is applied, the correctness and completeness rates are both increased. The average rate of false detected tree segments in the plot $\mathrm{A}, \mathrm{B}$ and $\mathrm{C}$ amount to $0.23,0.33$ and 0.31 , respectively. Note that the method is evaluated by the single trees located in the upper canopy layer.

Figures 4.10 and 4.11 show examples of the segmentation results by primitives obtained from mean shift clustering for a part of test plot A. Note that the sample plot contains only coniferous trees. The results indicate that our method was successful in overcoming the over- and under-segmentation problems in the test plot. Fig.4.10 presents the comparison of segmentation results between the normalized cut combined with mean shift clustering and the adaptive stopping criterion method. The tree clusters are classified using fitted elliptic paraboloids and the spatial overlap ratios between them are calculated. The red box in Fig.4.10a shows an over-segmented cluster which is delineated as a single tree (see Fig4.10b) by using the adaptive segmentation approach.

Moreover, the case of under-segmentation is indicated by Fig.4.11. In this case, we used the adaptive stopping criterion method to reduce the undersegmentation error of the normalized cut algorithm with the clusters obtained 
from the mean shift step. In Fig.4.11a, the red box is focused on an example cluster consisting of multiple trees, which is known as under-segmentation. Using the adaptive segmentation method, it is revealed in Fig.4.11b how the under-segmentation for the current cluster is removed.

The computational cost for both the static and the adaptive versions were similar, which indicates that the processing time was dominated by solving the generalized eigenvalue problem on the NCut similarity matrix. A simple heuristic may be used to reduce the number of times the adaptive stopping criterion is invoked: for a given point cluster, if its 3D bounding box exceeds the dimensions of the largest possible single object (i.e. tree), the segmentation must continue. In principle our approach is applicable for the larger forest areas.

Our method takes the advantage of fitting the elliptic paraboloids to the point clouds and determining whether the currently processed cluster of points represents a single coniferous tree or multiple trees. The sensitivity analysis shows that for all the sample plots the same set of parameters as maximum overlap ratio $\max _{o p_{r}}$ and minimum probability threshold $\min _{p_{t h r}}$ achieves the best trade off values between the correctness and completeness. The restrictions of the current approach are: (i) mainly trees in upper canopy layer in the 3D point cloud can be segmented accurately, (ii) the method fails in the plots with concentrations of deciduous trees where the segments' points cannot be clearly clustered. The crown boundaries of deciduous trees are not clear in the point clouds due to the complex geometry of these trees in the leaf-on condition. Therefore, no benefit was achieved despite the adaptive segmentation approach for the deciduous trees. In case that the study area is dominated by coniferous trees, single tree segments are successfully delineated by the classifier training and elliptic paraboloids fitting method. Moreover, the normalized cut threshold $N C u t_{t h r}$ has no physical meaning, however our method's main control parameters are closely tied with the appearance of single trees in the forest scene, which makes them more easily interpretable. 

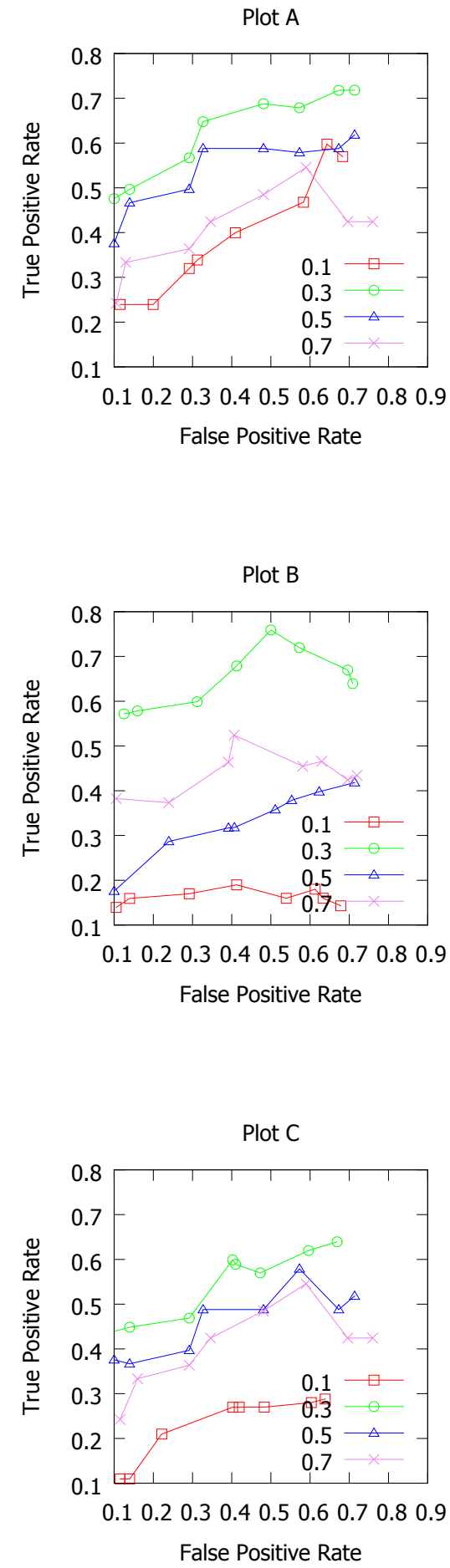

76 Figure 4.8: ROC curve of single tree adaptive segmentation for the plots A $(100 \%$ coniferous $)$, B (99\% coniferous) and C (70\% coniferous). Each diagram contains 4 ROC curves which correspond to various thresholds of the maximum overlap ratio $\max _{o p_{r}}$. 

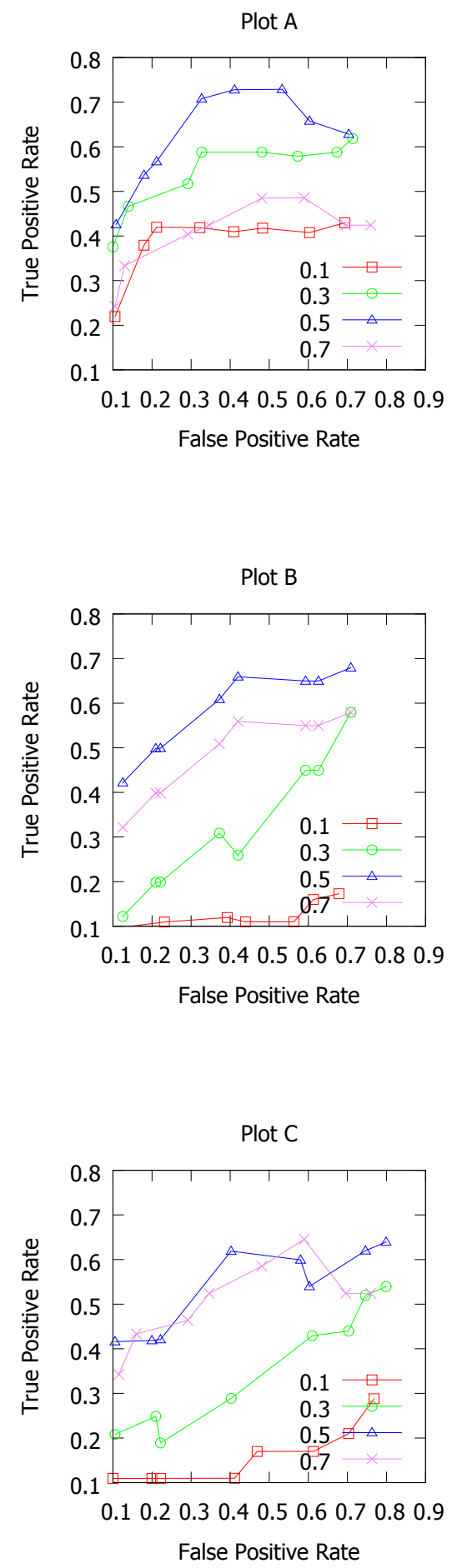

Figure 4.9: ROC curve of single tree adaptive segmentation for the plots A ( $100 \%$ coniferous), B (99\% coniferous) and C (70\% coniferous). Each diagram contains 4 ROC curves which correspond to various thresholds of the minimum probability threshold $\min _{p_{t h r}}$. 


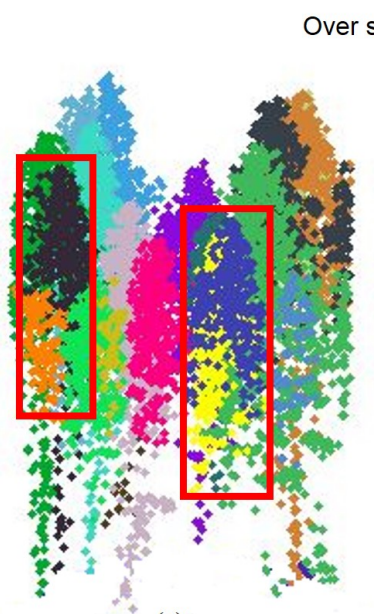

(a)
Vver segmentation

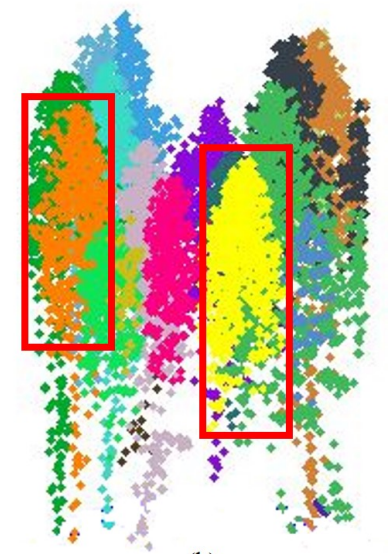

(b)

Figure 4.10: Single tree segmentation results for a part of plot A: (a) corresponds to the normalized cut segmentation with mean shift clustering results; and (b) represents the adaptive stopping criterion for normalized cut segmentation by fitting paraboloids, respectively. Each set of colored points represents a delineated single tree. The red boxes outline the over-segmentation issue on an example single tree.

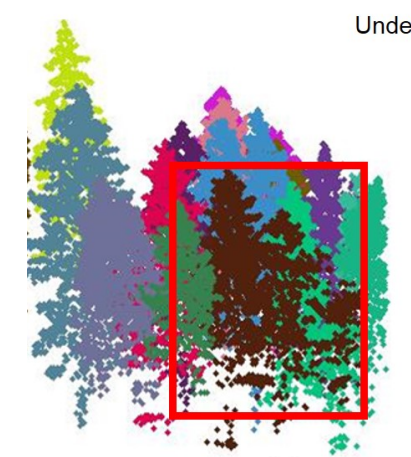

(a) Under segmentation

Figure 4.11: Single tree segmentation results for a part of plot A: (a) corresponds to the normalized cut segmentation with mean shift clustering results; and (b) represents the adaptive stopping criterion for the normalized cut segmentation by fitting paraboloids, respectively. Each set of colored points shows a delineated single tree. The red boxes outline the under-segmentation issue on an example group of single trees. 


\subsection{Conclusions}

The study presents a novel method for single tree segmentation in temperate coniferous forest by applying an adaptive stopping criterion to top-down segmentation in ALS point clouds. Following the study on segmentation of fallen stems (Polewski et al., 2015b), our results also confirm that the use of an appearance based stopping criterion can benefit a top-down segmentation process in different scenarios. Our method is directly applied to the 3D ALS point clouds, targeting coniferous trees through modeling their crowns by elliptic paraboloids. The adaptive segmentation approach on average appears to lead to an improvement of up to $10 \%$ in both correctness and completeness. We did not try to include the tree species composition in the analysis; this will constitute a future research issue. Moreover, the accuracy of the segmentation was negatively impacted by the higher number of deciduous trees in the upper canopy layer.

Further improvements to the method would be achieved by extending the adaptive segmentation approach to the deciduous tree species to deal with the over- and under-segmentation problems. Although some deciduous tree species may follow a well defined geometric crown shape, it is challenging to propose a single model which can accurately represent all broad-leaf trees. Moreover, higher point density ALS data as well as data acquisition in leaf-off condition could lead to more precise reconstruction of the single tree segments. Furthermore, we would like to utilize the stems obtained from the proposed method by Amiri et al. (2017) to enhance the single tree segmentation in the intermediate and lower canopy layers by providing prior knowledge about tree locations. 



\section{Tree species classification by fusing multispectral lidar and aerial imagery}

This chapter is based on:

Amiri, N., Heurich, M., Krzystek, P., \& Skidmore, A. K. (2018). Feature Relevance Assessment Of Multispectral Airborne Lidar Data For Tree Species Classification. International Archives of Photogrammetry and Remote Sensing, (Vol. XLII-3, pp. 31-34). International Society for Photogrammetry and Remote Sensing (ISPRS). DOI: 10.5194/isprs-archivesXLII-3-31-2018.

Amiri, N., Krzystek, P., Heurich, M., \& Skidmore, A. K. (Revised manuscript submitted). Tree species classification by fusing multispectral lidar and aerial imagery. Submitted to: ISPRS Journal of Photogrammetry and Remote Sensing 


\section{Abstract}

Forest inventory and management requires precise and detailed information about the distribution of tree species. While individual tree-based approaches using single wavelength lidar can successfully distinguish broad-leaf and coniferous trees, they cannot conduct more detailed tree species classification due to limited spectral ranges. Recent advances in sensor technology have led to the development of new Multispectral Laser Scanning (MSLS) systems that provide up to three different wavelengths. Fusing these MSLS data with multispectral aerial imagery significantly increases the radiometric range of the dataset for classifying multiple tree species. Thus far, the status and potential uses of MSLS data for classifying tree species have not been fully explored. In this study, different feature sets were extracted from an MSLS point cloud and multispectral aerial imagery to classify three tree species (Norway spruce, European beech, Silver fir), and dead spruce trees with crowns (snags) in the Bavarian Forest National Park, Germany. The MSLS data were acquired by combining data from three different sensors under leaf-on conditions with an average point density of 37 points $/ m^{2}$. First, the combined 3D point cloud was segmented into 3D clusters using the Normalized Cut segmentation approach. Second, various features from the MSLS point cloud and aerial imagery were generated. Third, forward stepwise feature selection was conducted to reduce the number of redundant or irrelevant features. Finally, the classification was conducted using multinomial logistic regression. We tested our classification procedure using 20 sample plots with measured reference single trees. The results for the individual MSLS point cloud and aerial imagery datasets showed that cross-validated (15-fold) accuracies of $78 \%$ and $66 \%$ were achieved, respectively. An improvement of 7-13\% over single wavelength approaches were achieved when the MSLSS data are used. However, there was no considerable improvement in the classification accuracy if the multispectral imagery features were fused with the MSLS data features. Overall, the contribution of the MSLS point cloud radiometric features to the classification accuracy was higher than that of the geometric features by approximately $10 \%$. Our results show that the features derived from a MSLS point cloud have a great potential to improve detailed tree species mapping. 


\subsection{Introduction}

Remote sensing can provide valuable information for understanding ecosystem structures and functions over large areas (Baldeck et al., 2015) that influence biodiversity (Turner et al., 2003). Aside from area-based approaches (Korpela et al., 2009), a large number of studies have mentioned the fundamental role of species identification at the single tree level in a wide range of forest inventory and management activities (Treitz and Howarth, 2000; Heinzel and Koch, 2012; Fassnacht et al., 2016) as well as biodiversity monitoring (Skidmore and Pettorelli, 2015). Therefore, in order to maintain up-to-date information, effective methods and techniques need to be developed that accurately classify single tree species.

Single tree species can initially be identified using individual tree detection approaches and later mapped by a classification strategy. Recent innovative methods for single tree detection have utilized a 3D approach instead of using the canopy height model (CHM) alone to resolve the over/under-segmentation problems (Wang et al., 2008). The detection rates for single trees can be improved significantly by applying the spectral clustering Normalized Cut method (NCut) to a (super) voxel forest structure (Reitberger et al., 2009; Yao et al., 2013), and introducing a classifier-based adaptive stopping criterion (Amiri et al., 2018b). Moreover, to segment individual trees Strîmbu and Strîmbu (2015) proposed an approach that captures the topological structure of the forest in hierarchical data structures and quantifies the tree crown component relationships in a weighted graph. Overall, accurate single tree segmentation is an important step for high quality species determinations at the individual tree level.

\subsection{Related work}

Over decades, optical imagery that can remotely measure the spectral reflectance of an object has been used as a standard source to discriminate tree species (Erikson, 2004; Fassnacht et al., 2016). Optical aerial instruments can record the spectral signatures of tree species not only in the visible spectral range (RGB), but also in the near-infrared (NIR), short-wave infrared (SWIR), and even thermal infrared. Depending on the radiometric resolution, the radiation can be measured in multiple bands. Multispectral sensors typically provide up to 10 spectral bands, whereas hyperspectral sensors have hundreds of bands. Recently, dense matching has become a mature technique used to reconstruct objects from a series of highly overlapping images on the pixel level with excellent subpixel accuracy (Hirschmüller, 2008). Regarding forestry applications, this novel computer vision method enables a dense point cloud to be generated from canopy surfaces that later can be used for tree species classification either on the tree level, or using an area-based approach. Moreover, multispectral and hyperspectral sensors can be combined with lidar to enrich the limited radiometric information of lidar. Ullah et al. (2017) demonstrated that estimating forest structural parameters at the stand and forest compartment level can be improved by 
using point clouds generated from aerial imagery. Nevalainen et al. (2017) combined an RGB camera and a frame format hyperspectral camera mounted on a copter drone. They showed that the single tree detection rate is strongly dependent on the boreal forest stand characteristics and ranges between $40 \%$ and $95 \%$. Moreover, the tree species classification accuracy (four different tree species) achieved an overall accuracy of $95 \%$ and a F-score of 0.93 , respectively. However, the main drawback of these passive sensors for forest applications is the limited forest canopy surface penetration, hence, the forest structure beneath the canopy cannot not be fully captured in $3 \mathrm{D}$.

Over the past decade, Airborne Laser Scanning (ALS) point clouds have become beneficial for classifying tree species. Several studies have proposed using structural features from the ALS point clouds, such as crown shapes, height distribution percentiles, and proportions of first/single returns for distinguishing between tree species (Brandtberg, 2007; Holmgren et al., 2008; Ørka et al., 2009; Lindberg et al., 2015). Separating trees by height is important for single tree classification, especially in forests where tree height distributions differ between species (Holmgren and Persson, 2004; Brandtberg, 2007). After the advent of single full waveform lidar systems, several studies reported accuracy improvements by applying waveform features that use detailed backscattered pulse information, such as the intensity and pulse width (Shang and Chazette, 2014; Hovi et al., 2016). Höfle et al. (2008) used calibrated waveforms from the ALS data to distinguish between European larch, English oak, durmast oak, and European beech, and found that echo width could separate larch from broad-leaf trees. However, the responses of the oak and beech represented by the backscatter cross-section and echo width were similar. Reitberger et al. (2009) found that radiometric information derived from full waveform lidar, such as the intensity and pulse width, provide a strong basis for distinguishing between broad-leaf and coniferous trees. Heinzel and Koch (2011) explored a set of waveform-based features for classifying four groups of tree species in a mixed temperate forest with an overall accuracy of $78 \%$. Yao et al. (2012) found that single wavelength ALS data $(1550 \mathrm{~nm})$ could be used to classify coniferous and broad-leaf trees in the Bavarian Forest National Park with a maximum overall accuracy of $90 \%$. However, Shi et al. (2018a) found that the classification accuracy decreases by $30 \%$ if the detailed tree species mapping (six different tree species) is attempted for the same study area. Further, Hovi et al. (2016) focused on systematically analyzing the identification potential of ALS point cloud features by investigating the sources of the within-species variations. They achieved an overall accuracy of $75 \%$ for the identification of three main tree species in Finland using lidar waveform features. Overall, considering the limitations of optical imagery, single wavelength ALS point clouds (full waveform) are superior data sources for classifying tree species (Korpela et al., 2010b). However, due to the lack of spectral information, detailed tree species identifications have yet to reach sufficiently high accuracies (up to 90\%) (Leckie et al., 2003; Yu et al., 2017).

The aforementioned results suggest that the intensity of single wavelength lidar (full waveform) is useful for classifying tree species. Moreover, the reflectivity of each object is dependent on the laser wavelength. For instance, 
according to Kim (2008), by using single wavelength lidar (1064 nm) under leaf-on conditions, the average intensity values of broad-leaf trees are higher than those of most coniferous tree species. This difference is mainly due to differences in the tree structures. Broad-leaf trees have larger single leaves, while coniferous trees have needles with a non-continuous leaf surface (Kim et al., 2009). Recently, Shi et al. (2018a) verified that the intensity features make a more significant contribution to tree species classification in mixed temperate forests than the geometric features. Moreover, the bidirectional reflectance and geometry of the volumetric target surfaces significantly influence the intensity values recorded by a lidar system (Fassnacht et al., 2016).

Integrating the two different (active and passive) remote sensing approaches combines the advantages of each method and resolves some of the disadvantages when they are used alone. Previous studies reported that integrating single wavelength ALS data with hyper/multispectral imagery can improve the accuracy of tree species classification by at least 10\% (Trier et al., 2018). Holmgren et al. (2008) classified three tree species in a boreal forest and achieved an overall accuracy of $96 \%$ when multispectral imagery and ALS data were combined. Dalponte et al. (2012) combined lidar and hyperspectral data for the classification of tree species using Random Forest in the southern Alps and achieved an overall accuracy of $74.9 \%$. However, some studies have reported serious commission errors mostly caused by ground vegetation illuminated by the sun that were subsequently recorded by the optical imagery (Leckie et al., 2003). Furthermore, geometric mis-registration can limit the effective performance of the combined datasets (Yu et al., 2017). Recently introduced MSLS technology is promising for improving forest mapping as it can provide a denser point cloud and higher spectral information. Few studies have focused on the potential of using MSLS point clouds for classifying tree species (Lindberg et al., 2015; St-Onge and Budei, 2015; Hopkinson et al., 2016; Yu et al., 2017; Axelsson et al., 2018; Budei et al., 2018). Lindberg et al. (2015) generated MSLS data using three different instruments during different flights with a point density of 20 points $/ m^{2}$ to characterize tree species. They employed visual interpretation to show that, if both spectral and geometric information from multi-wavelength lidar data are used, the tree species identification performance is better than that obtained when information from single wavelength ALS data are used. St-Onge and Budei (2015) used the intensity-based features extracted from three spectral channels of a Titan multispectral lidar system (Optech, 2015) to classify broad-leaf vs. needle-leaf trees in a Canadian boreal forest and achieved a classification accuracy over 90\%. Yu et al. (2017) used the same sensor and achieved an overall tree species classification accuracy of $85.6 \%$ for 3 different tree species in southern Finland using intensity-based features. Hopkinson et al. (2016) compared terrain and forest canopy attributes extracted from each wavelength of two multispectral lidar datasets (multisensor and singlesensor). They achieved an overall accuracy of $78 \%$ for the classification of land surface and vegetation ( 8 classes) by integrating spectral and structural information. Axelsson et al. (2018) used the Optech Titan $X$ System to investigate ten tree species in a boreal forest, and achieved a cross-validated 
accuracy of $76.5 \%$ using the height and intensity distribution of features from the tree segments. Budei et al. (2018) classified 10 tree species using the Optech Titan system with an overall accuracy of $75 \%$. So far, combinations of various features from MSLS point clouds have been mainly used to examine tree species classification in boreal forests.

In summary, the classification of coniferous and broad-leaf trees with single wavelength ALS data (full waveform) is possible with high accuracy. Furthermore, the fusion of single wavelength ALS data with hyper/multispectral imagery improves the tree species classification accuracies. Therefore, from a research point of view, it is meaningful to determine if combining multispectral data from lidar and aerial imagery significantly improves the classification accuracy in temperate forests. Moreover, due to the expected high dimensional feature space, techniques are mandatory to reduce the huge number of predictive variables to the most prominent ones.

The main objectives of this study were to evaluate the accuracy of tree species classification in a temperate forest located in southwest Germany using (i) multispectral aerial imagery features, (ii) MSLS point cloud features, (iii) combined features from both datasets, and (v) single spectral channel (1550 $\mathrm{nm}, 1064 \mathrm{~nm}$, and $532 \mathrm{~nm}$ ) features. Additionally, the classification performance of the combined features extracted from each spectral channel (1550 $\mathrm{nm}, 1064 \mathrm{~nm}$, and $532 \mathrm{~nm}$ ) and aerial imagery was assessed. Furthermore, we assessed the classification accuracy and identified the most important features based on a specific feature selection approach.

The remainder of the paper is structured as follows: Section 3 illustrates the study area, materials, and field measurements. Section 4 describes the details of our approach. The results are presented and discussed in Section 5. Finally, the conclusions are stated in Section 6 .

\subsection{Experiment}

\subsubsection{Materials}

Located in the Bavarian Forest National Park, our study area is a temperate and complex forest situated in the south-eastern part of Germany along the Czech Republic border covering an area of 24,250 ha. The forest is dominated by Norway spruce (Picea abies) with European beech (Fagus sylvatica) and Silver fir (Abies alba). Rare tree species are also present in the park, such as white birch (Betula pendula), sycamore maple (Acer pseudoplatanus), and common rowan (Sorbus aucuparia) (Cailleret et al., 2014). Fig.5.1 shows in yellow the sample plots site where the experiments were carried out on a color infrared orthophotograph of the Bavarian Forest National Park.

Acquisition of the multispectral aerial imagery (color infrared) was conducted on June 23, 2016 under leaf-on conditions over the Bavarian Forest National Park using a DCM camera with a ground sample distance of $20 \mathrm{~cm}$. Additionally, Global Positioning System (GPS) and Inertial Navigation System (INS) data were acquired to provide initial information about the exterior orientation. The mean above-ground flight height was $2918 \mathrm{~m}$. The images 


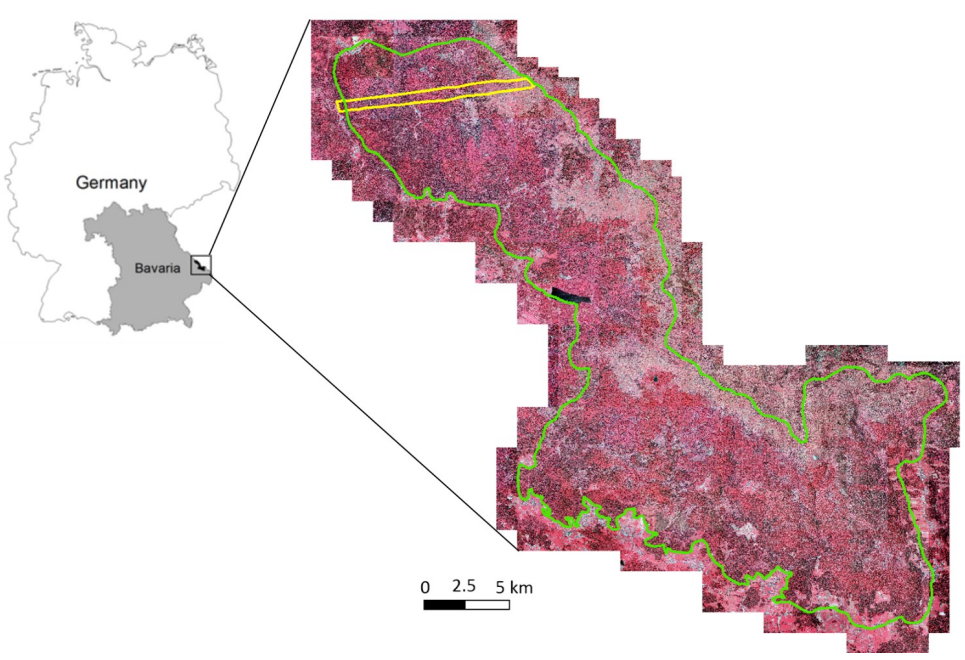

Figure 5.1: Color infrared orthophotograph of the Bavarian Forest National Park (park area in green), with the test area containing sample plots for classifying tree species marked in yellow.

had an end-lap of $75 \%$ and side-lap of $60 \%$. They contained three spectral bands $c h$ : near-infrared nir, red $r$, and green $g$. The aerotriangulation was calculated using the Trimble/INPHO ${ }^{\complement}$ software using as input the aerial images, the camera calibration model, and the image measurements for the control points. The Trimble/INPHO ${ }^{\complement}$ software also generated orthophotos utilizing the digital surface model of the forest area. Radiometric corrections were later applied to the orthophotos. A color infrared image of the study area that presents the difference between the trees (living vegetation) and snags (standing dead spruce trees with crowns) by color, is shown in Fig.5.2.

Table 5.1: Flight campaigns of multispectral lidar data.

\begin{tabular}{llll}
\hline Riegel scanner type & LMS-680i & LMS-Q780 & VQ-880-G \\
\hline Platform type & Airplane: & Airplane: & Airplane: \\
& D-HALL/AS350 & Tecnam P2006T & Tecnam P2006T \\
\hline Spectral wavelength $(\mathrm{nm})$ & 1550 & 1064 & 532 \\
\hline Beam divergence $(\mathrm{mrad})$ & 0.5 & 0.25 & 0.2 \\
\hline Flight speed $\left(\mathrm{m} / \mathrm{s}^{-1}\right)$ & 50 & $90-100$ & $90-100$ \\
\hline Flight height $(\mathrm{m})$ & 300 & 550 & 550 \\
\hline Footprint size $(\mathrm{mm})$ & 150 & 137 & 110 \\
\hline
\end{tabular}

The main goal of the experiment was to combine three different full waveform lidar sensors that each provided a specific spectral wavelength. The multispectral lidar data were acquired on August 18, 2016 during leaf-on conditions using three Riegl scanners: LMS-680i, LMS-Q780, and VQ-880-G. Note that the weather conditions remained constant during data acquisition. The combined $3 \mathrm{D}$ point cloud with an average point density of $37 \mathrm{point} / \mathrm{m}^{2}$ contained 


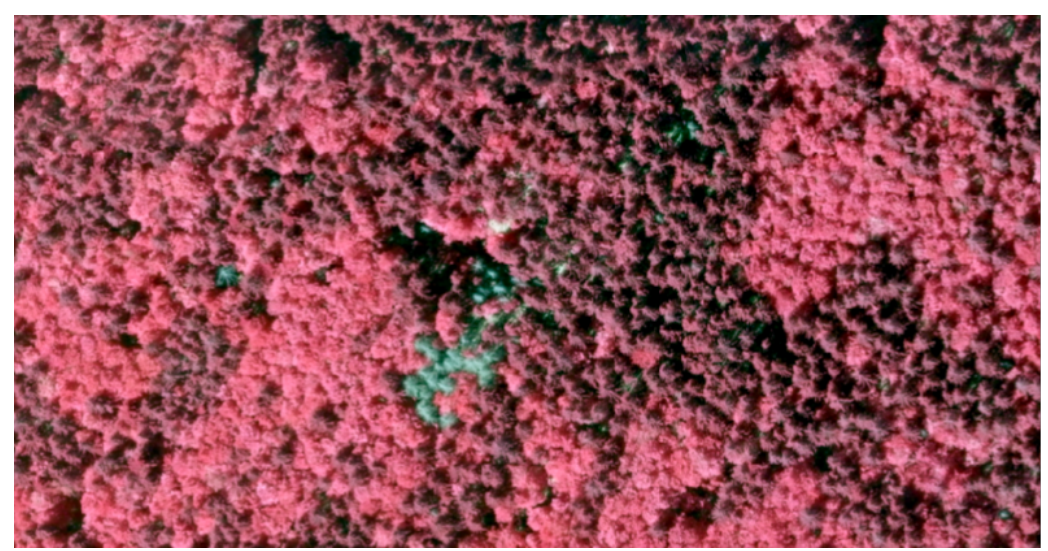

Figure 5.2: A forest scene in the color infrared aerial image with a ground pixel resolution of $20 \mathrm{~cm}$. Snags (standing dead spruce trees with crowns) appear in gray, while the living trees are shown in red (broad-leaf and coniferous trees).

three different spectral channels (1550 nm (Ch1), $1064 \mathrm{~nm}(C h 2)$, and 532 $\mathrm{nm}(C h 3))$. Table 5.1 contains the acquisition flight details.

The VQ-880-G and LMS-Q780 sensors were flown in an one airplane, while the LMS-680i instrument was carried in another airplane. The MSLS data are visualized in Fig.5.3. Prior to the segmentation step (see subsec.5.4.3), the spectral $C h 2$ and $C h 3$ channels were horizontally and vertically shifted to the $C h 1$ reference channel. The reference channel had been geometrically calibrated in advance based on vertical and planimetric objects, such as enclosed building polygons and flat areas, respectively.

\subsubsection{Reference data}

The ground truth data for the sample plots were acquired by field measurements and included 586 single trees that were measured from 20 circular sample plots with an area of $500 \mathrm{~m}^{2}$ for each plot. The reference trees positions (all trees with DBH (Diameter at Breast Height) $>7 \mathrm{~cm}$ ) were measured from the center of the plot with a vertex for the distance and a compass for the angle using the Leica GS GPS system (see Bässler et al. (2008)). Moreover, the DBH values for all the trees and heights of all trees were measured. The measurement campaign was conducted during the summer of 2017. Based on the available ground truth data and, due to the lack of reference data for some of the rare species, we selected three species for the study: Norway spruce (Picea abies), European beech (Fagus sylvatica), and Silver fir (Abies alba). Additionally, we added snags (standing dead spruce trees with a crown) to the list of tree classes to be identified. The distribution of the four tree classes in the sample plots for the classification were as follows: Norway spruce (43\%), European beech (30\%), Silver fir (10\%), and snags (16\%).

The reference data were unbalanced and dominated by beech and spruce 
trees. Therefore, the reference data were balanced for the classifier and the experiment was run 20 times by randomly selecting a subset of the dominant class each time so that the reference data contained no more than $32 \%$ of any class. The single trees were subdivided into three canopy layers with respect to the top tree height $h_{\text {top }}$ in the plot that was defined as the average height of the 100 tallest trees per ha (Van Laar and Akça, 2007; Heurich, 2008). We focused on the upper canopy layer that contained trees with heights that were at least $80 \%$ of the $h_{\text {top }}$ and could be identified in the reference data using a matching strategy.

For matching, we selected the aforementioned tree classes in the upper canopy layer from the reference data and linked them to the correctly segmented single trees by conducting the following procedure. We followed the strategy proposed by (Reitberger, 2010) to match the reference and segmented single trees. The single tree positions from the Normalized Cut segmentation (NCut) (see subsec.5.4.3) were matched with the reference trees if, (a) the distance from the center of a segment to the reference tree was smaller than $60 \%$ of the mean tree distance of the plot, and (b) the height difference between $h_{t o p}$ and the height of the reference tree was smaller than $20 \%$ of $h_{t o p}$ (Heurich, 2008). If a reference tree was assigned to more than one tree position detected by the NCut segmentation, the tree position with the minimum distance to the reference tree was selected, otherwise, it was removed from further analysis. The matched 3D tree segments in the upper canopy layer were extracted and assigned to the corresponding reference trees for all sample plots. The final number of the matched tree segments with the reference data in the upper canopy layer that were used for further classification analyses are summarized in Table5.2.

Table 5.2: The number of matched trees in the upper canopy layer with the overall tree species percentage in the plots.

\begin{tabular}{lll}
\hline & Tree species (\%) & Number of trees \\
\hline Norway spruce & 35 & 161 \\
\hline European beech & 30 & 138 \\
\hline Silver fir & 15 & 67 \\
\hline Snag & 18 & 82 \\
\hline
\end{tabular}

\subsection{Method}

\subsubsection{Pre-processing of lidar data}

A full waveform lidar system provides the reflected digitized (typically 15 $\mathrm{cm}$ ) waveform at regular intervals and includes information regarding the reflected intensity and pulse width. An appropriate waveform decomposition is required to obtain these parameters. By using superimposed Gaussian functions, the $3 \mathrm{D}$ coordinates $\left(x_{v}, y_{v}, z_{v}\right)$ of each reflecting object $v$ hit by the laser pulse were obtained along with the intensity $I_{v}$ and pulse width $P W_{v}$ as physical properties (Reitberger et al., 2009). Overall, this 
decomposition generated a point cloud for the forest area represented by the vector $P_{n}\left(x_{n}, y_{n}, z_{n}, I_{n}, P W_{n}\right), n=1, \ldots, N$ ( $N$ is the total number of points in the point cloud) (Amiri et al., 2016). The intensity $I_{n}$ can be interpreted as the pulse energy and is equal to the area of a single Gaussian function. This value is dependent on the traveling distance $r_{n}$ (in one direction) and must be normalized with respect to a reference distance $r_{r e f}$ according to Eq.5.1 (Hopkinson, 2007; Briese et al., 2008; Reitberger et al., 2008).

$$
I_{n}^{c o r r}=I_{n}\left(\frac{r_{n}}{r_{r e f}}\right)^{m}
$$

The $m$ parameter could be estimated from lidar data acquired in a special calibration flight (Reitberger, 2010). In this study, we used the theoretical value $m=2$. Commercial software packages, such as Riegl RiAnalyze ${ }^{\complement}$ can provide the amplitude $a_{n}$ and/or the reflectance $\rho_{n}$. The latter is also often referred to as an intensity and refers to the fraction of incident optical power reflected by a target at a certain wavelength. The amplitude $a_{n}$ is defined by each hardware manufacturer (see Riegl (2012)). In Riegl scanners, the amplitude $a_{n}$ is defined as a linear measure for the pulse energy. The reflectance $\rho_{n}$ is often referred to extending Eq.5.1 and following the suggestions of Höfle and Pfeifer (2007), the range-independent reflectance $\rho_{n}$ can be approximately converted from the amplitude $a_{n}$ by the following formula (Riegl (2012), private communication (Riegl, Nov.2017)):

$$
\rho_{n}=\rho_{r e f}\left(\frac{a_{n}}{a_{r e f}}\right)\left(\frac{r_{n}}{r_{r e f}}\right)^{2} \eta_{a t m}
$$

Eq.5.2, refers to an instrument whose emitted laser beam that perpendicularly hits a target area of $100 \%$ reflectance $\left(=\rho_{\text {ref }}\right)$ and measures at a distance of $r_{r e f}$ and an amplitude of $a_{r e f}$. The term $\eta_{a t m}=\exp (0.0000978 \times 2 \times$ $\left.\left(r_{n}-r_{r e f}\right)\right)$ describes the atmospheric attenuation (the loss of energy through the scattering and absorption of photons from the laser beam $(1550 \mathrm{~nm})$ in the atmosphere) is assumed to cover a visual range of $23 \mathrm{~km}$ (Reitberger, 2010). Note that the reflectance $\rho_{n}$ values are also affected by the angle of incidence (the angle between the emitted laser beam and the target surface normal)(Kashani et al., 2015). However, we assumed that the incident angle was unknown and thus, this effect has been neglected.

The $\rho_{n}$ reflectance values were calculated from the LMS-Q780 and VQ-880-G scanner data using the Riegl RiAnalyze ${ }^{\circledR}$ software. By default, these values were corrected considering the traveling distance $r_{n}$. However, for the LMS680i scanner data, the Riegl RiAnalyze $\left.{ }^{(}\right)$software could only provide the amplitude values. Therefore, the amplitude $a_{n}$ for each reflection point $P_{n}$ was approximately converted to the reflectance $\rho_{n}$ using Eq.5.2. In order to obtain reasonable values for the parameters $a_{\text {ref }}$ and $r_{r e f}$, a sample of the amplitude $a_{n}=165$ in Eq.5.2 was taken as the mean value from four small concrete areas $(4 \times 4 \mathrm{~m}$ ) located on an airfield (see Fig.5.4). Each area was selected in the nadir of four different lidar strips that had approximately the same flying height $r_{n}=420 \mathrm{~m}$. Finally, we defined that the $a_{r e f}=250$ and 
(a)

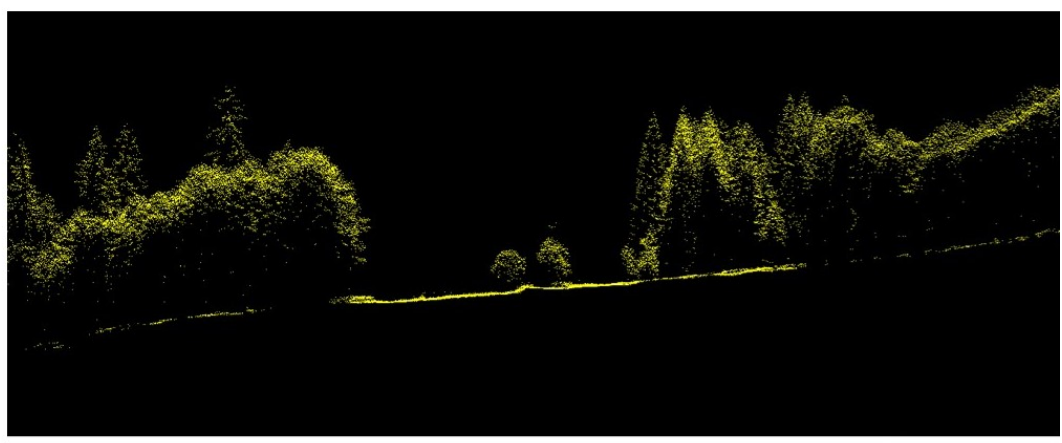

(b)

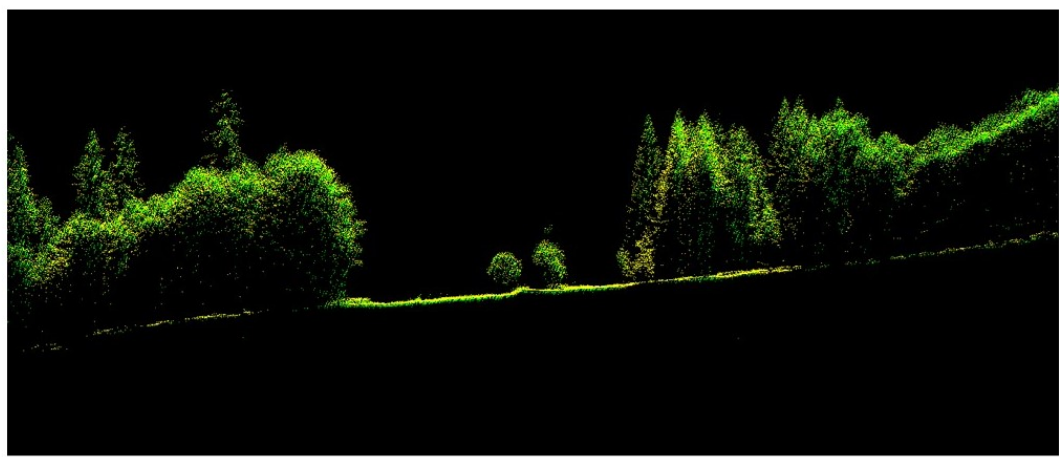

(c)

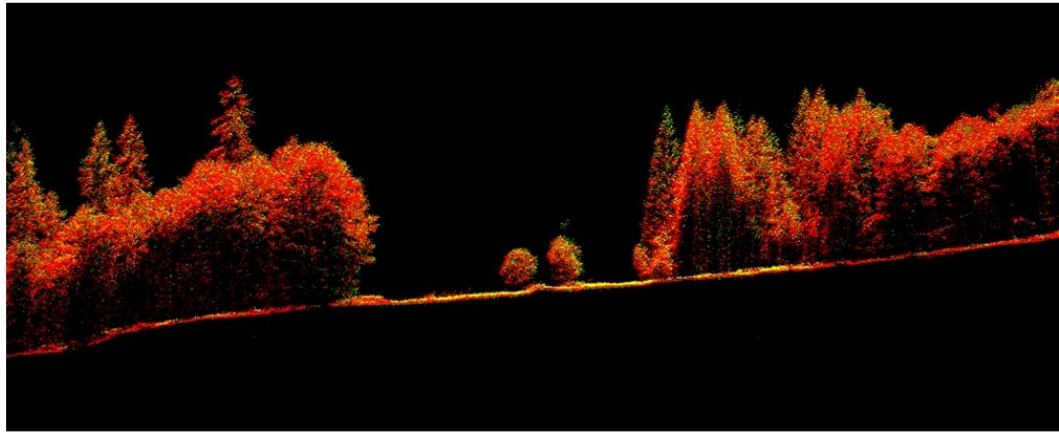

Figure 5.3: Vertical profile of a forest scene in the 3D point cloud; a) $532 \mathrm{~nm}$ spectral wavelength, b) addition of the $1064 \mathrm{~nm}$ wavelength, and c) the 1550 $\mathrm{nm}$ wavelength is combined with the 532 and $1064 \mathrm{~nm}$ wavelengths.

$r_{\text {ref }}=600 \mathrm{~m}$ parameters should refer to a reflectance of $\rho_{\text {ref }}=100 \%$. This in turn means that according to Eq.5.2, the amplitude $a_{n}=165$ is equivalent to a reflectance of $\rho_{\text {ref }}=31 \%$. After the pre-processing step, a final visual inspection of the overlaying lidar strip areas showed there were no tiling effects.

\subsubsection{Outline of method}

The method used in our study was as follows. We segmented the lidar point cloud (MSLS and single wavelength) into 3D segments representing 


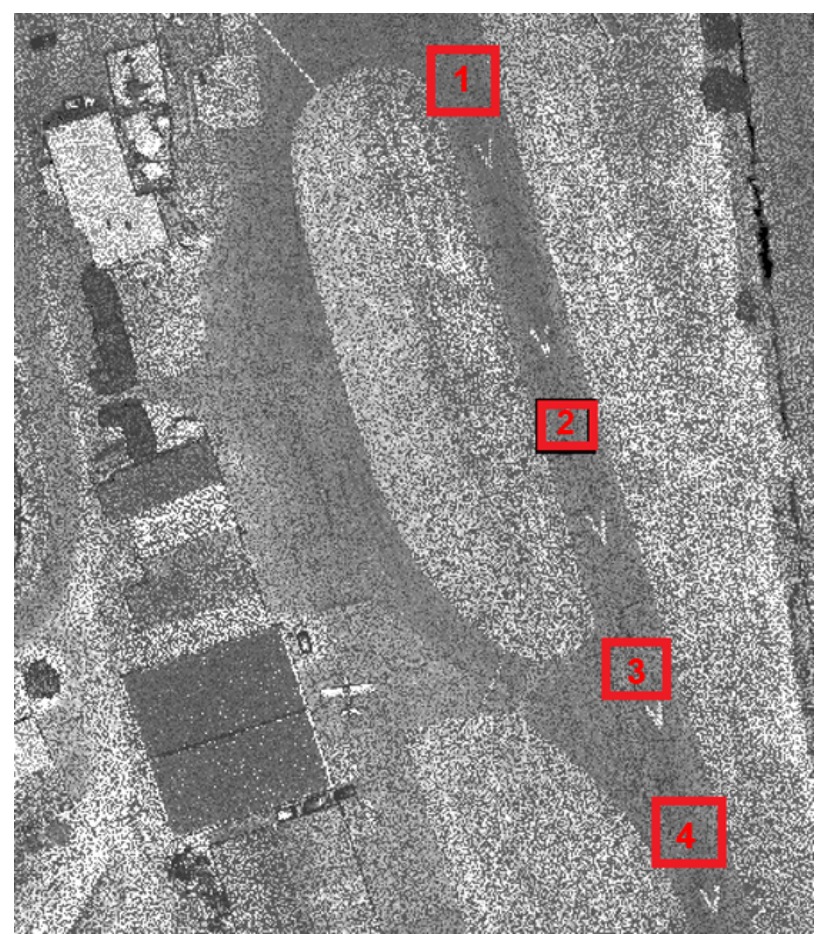

Figure 5.4: The four selected small target areas located on the airfield that were used for the amplitude calibration $\left(a_{r e f}\right)$.

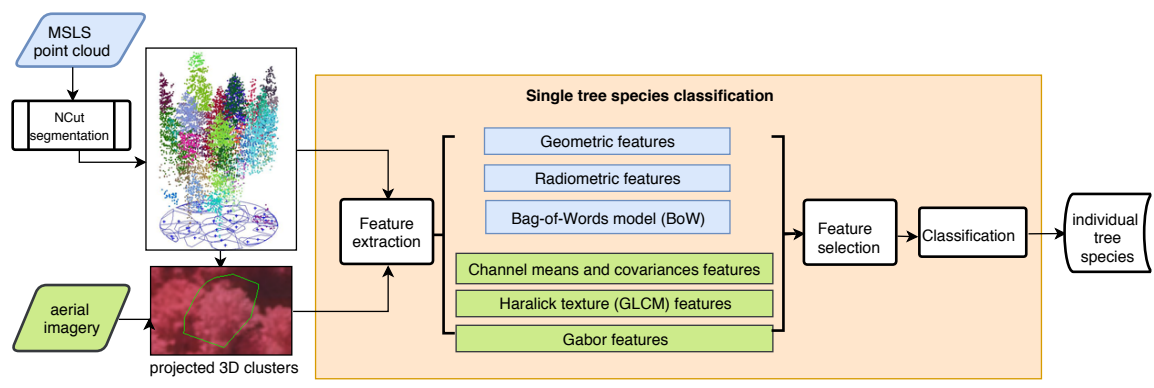

Figure 5.5: Overview of the feature-based strategy for tree species classification.

single trees using the Normalized Cut (NCut) algorithm (Shi and Malik, 2000; Reitberger et al., 2009) (see subsec.5.4.3) after performing the above mentioned pre-processing step (see subsec.5.4.1). The procedure recursively partitioned the 3D input data, beginning with the entire point cloud until the level representing single trees was achieved. The aim of the segmentation step was to divide points in the cloud with similar attributes into homogeneous 3D segments (Amiri et al., 2018b). We regarded this phase as an external procedure and the tree species classification step was our main focus. After 
the $3 \mathrm{D}$ point cloud was segmented, the convex hulls of each segment were projected onto the image plane to generate $2 \mathrm{D}$ polygons. As the coverage of the single trees in the upper canopy layer was better, we only considered the tree crowns that were visible from the top that could be identified in the reference data using the matching strategy (see subsec.5.3.2). We then extracted features in both the $2 \mathrm{D}$ image raster and in the $3 \mathrm{D}$ point cloud spaces. The forward stepwise selection method was applied to extract the most significant features for the tree species identification. The final selected features were used in a multinomial logistic regression for classification. A schematic of the entire processing procedure is presented in Fig. 5.5. This method is explained in detail by the following subsections.

\subsubsection{Normalized Cut segmentation}

The Normalized Cut algorithm (Shi and Malik, 2000) is a top-down method for segmenting objects over a discrete graph structure $G=(V, E)$ where the vertices $V$ represent the individual objects and the edges $E$ correspond to the neighborhood topology. In order to construct a low-dimensional representation of the input 3D points as objects, the method uses the eigenvalues associated with the object similarity matrix (Polewski et al., 2015b). The goal is to partition the input 3D point clouds into disjointed segments where the Normalized Cut criterion is minimized. A recursive bisection of the graph's vertices $V$ into disjointed segments $A$ and $B$ was performed while maximizing the intra-segment similarity of the objects is equivalent to minimizing their inter-segment dissimilarity (see Reitberger et al. (2009)). The frequently applied Normalized Cut is:

$$
\operatorname{NCut}(A, B)=\frac{C u t(A, B)}{A \operatorname{ssoc}(A, V)}+\frac{C u t(A, B)}{A \operatorname{ssoc}(B, V)}
$$

where $\operatorname{Cut}(A, B)=\sum_{i \in A, j \in B} w_{i j}$ is defined as the total sum of the weights between the $A$ and $B$ segments, while $A \operatorname{ssoc}(A, V)=\sum_{i \in A, j \in V} w_{i j}$ is the sum of the weights of all the edges ending in segment $A$. The Normalized Cut segmentation is based on multiple control parameters whose values can be optimized experimentally. The Normalized Cut segmentation (NCut) is based on multiple control parameters whose values can be optimized experimentally. The main control parameter values for the Normalized Cut segmentation ( $N C u t$ ) that we used in our experiment are summarized in the Table 5.3.

Table 5.3: Main control parameters for the single tree segmentation using the Normalized Cut method.

\begin{tabular}{lll}
\hline Parameters & Symbols & Values \\
\hline Normalized Cut threshold & NCut & 0.16 \\
\hline $\begin{array}{l}\text { Minimum number of points } \\
\text { in a segment }\end{array}$ & Min $_{\text {num }}$ & 10 \\
\hline
\end{tabular}


Table 5.4: Description of the generated features for each tree segment $i$.

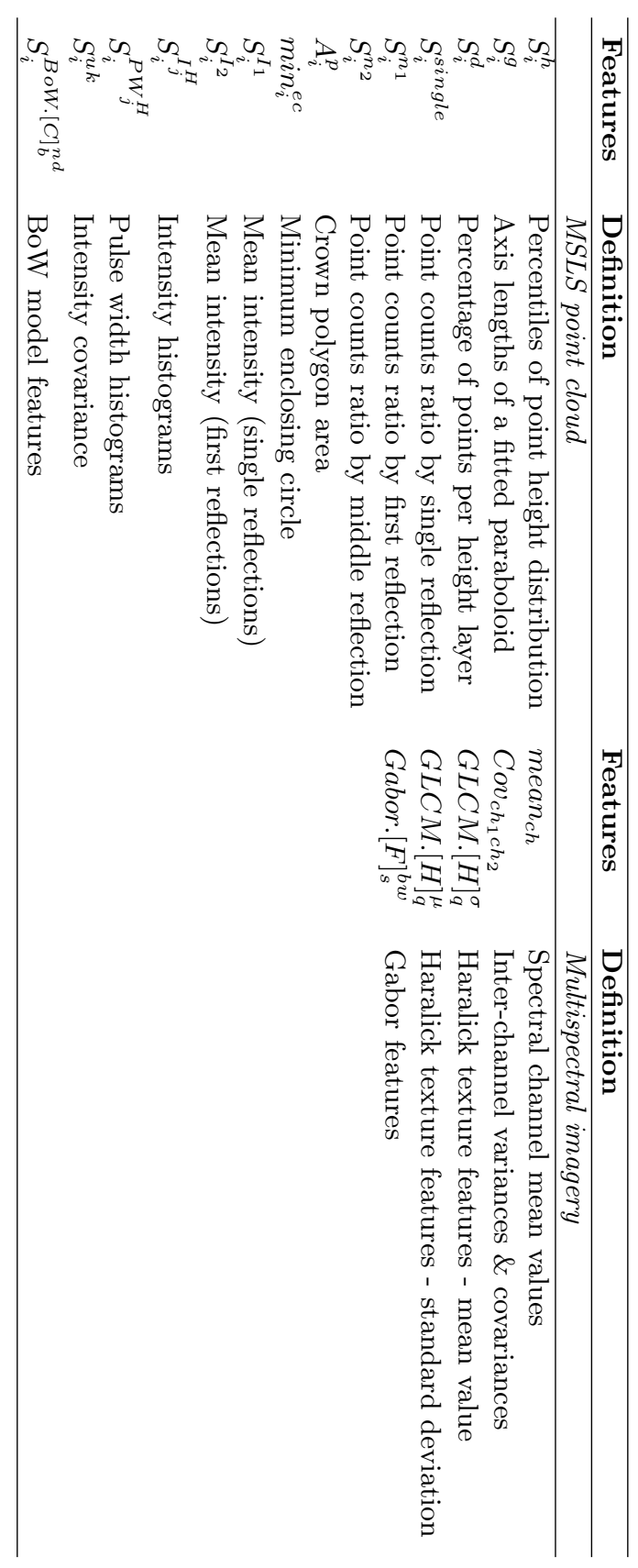

\subsubsection{Feature extraction}

The different feature sets derived from the multispectral aerial imagery and MSLS point cloud can be grouped into the categories presented below. All 
generated features are summarized in Table 5.4.

\subsubsection{Multispectral imagery features}

Values from the multispectral aerial imagery were extracted for each 3D tree crown polygon projected onto the image plane. These features can be grouped into the following three categories:

i) Channel mean values and covariance matrix:

The mean values of each spectral channel $c h$ over all pixels inside the $2 \mathrm{D}$ polygon mean $_{c h}$ and the six independent inter-channel variances and covariances $\operatorname{Cov}_{c h_{1}} \mathrm{ch}_{2}$ were extracted (ch refer to the near infrared nir, red $r$, and green $g$ bands (see subsec.5.3.1)).

ii) Haralick texture features:

In 1973, Haralick and Shanmugam introduced a popular statistical technique for extracting textural features (Haralick and Shanmugam, 1973). The Haralick texture features (GLCM) are computationally simple but efficient for many texture classification problems. In this study, first the images were converted to grayscale by taking the mean value of all the channels. For each tree segment, the minimum enclosing rectangle of the crown polygon (all pixels inside the rectangle) were used for GLCM calculations. Then, for each pixel a set of the 14 Haralick features $G L C M$. $[H]$ ( $H$ stands for contrast, correlation, variance, energy, entropy, homogeneity, and others) were calculated. In order to have each of the features rotationally invariant, the features were calculated for $K$ rotations that result in $K$ values from different image orientations. We used 4 orientations $\left(K=0^{\circ}, 45^{\circ}, 90^{\circ}\right.$, and $\left.135^{\circ}\right)$ to obtain the rotationally invariant values. A window size of $5 \times 5$ pixels was chosen. Furthermore, we introduced six levels of quantization $q$ $(2,4,8,16,32$, and 64$)$ in order to determine the optimal number of gray levels that would provide the most discriminative features for the classification task. Therefore, we tried different gray level quantizations because we did not know in advance which level would yield the optimal result. The subsec.5.4.5 feature selection step is capable of handling this increased number of features. Finally, per bounding box, the mean values $G L C M .[H]_{q}^{\mu}$ and the standard deviations $G L C M .[H]_{q}^{\sigma}$ of the 14 GLCMs after the $K$ orientations were calculated, respectively.

iii) Gabor features:

This model is an established method for representing high-level characteristics used for classification purposes (Toldo et al., 2010). The main aim of the Bag-of-Words (BoW) model is to approximate the feature vectors using a vector quantization algorithm with a set of prototypes. As proposed by Weinmann et al. (2015a), our BoW model $S_{i}^{B o W \cdot[C]_{b}^{n d}}$ contained eight geometric features generated from the local covariance matrix. In the BoW model, $C$ stands for the linearity, planar- 
ity, scattering, omnivariance, anisotropy, eigenentropy, sum of eigen values, and change of curvature that are commonly used in 3D ALS data processing (Weinmann et al., 2015b; Hackel et al., 2016). The frequency histograms for each feature were then constructed using 2-14 bins $b$ that generated a simple BoW model. This was conducted for seven spherical point neighborhood sizes with radii of $n d=0.2-1.6 \mathrm{~m}$ (0.1) that were later used for computing the covariance matrix. The radii later were divided into the three groups of $0.2 \mathrm{~m}, 0.3-0.8 \mathrm{~m}$ and 1.1-1.6 m. The optimal neighborhood radii $n d$ were defined using the tree crown diameters from the reference data to limit the generated feature count and prevent overfitting problems.

\subsubsection{MSLS point cloud features}

For each tree segment $i$, the following features $S_{i}=\left\{S_{i}^{h}, S_{i}^{g}, S_{i}^{d}, S_{i}^{\text {single }}, S_{i}^{n}, A_{i}^{p}\right.$ , $\left.\min _{i}^{e c}, S_{i}^{I^{H}}, S_{i}^{P W^{H}}, S_{i}^{I}, S_{i}^{B o W \cdot[C]_{b}^{n d}}, S_{i}^{u k}\right\}$ were extracted from the MSLS point cloud represented by $P_{n}\left(x_{n}, y_{n}, z_{n}, \rho_{n}, P W_{n}\right)\left(n=1, \ldots, N_{1}\left(N_{1}\right.\right.$ refers to the number of points in the tree segment $i))$. The features can be divided into three main groups:

i) Geometric features:

The features included: percentiles of the point height distribution in a tree segment $S_{i}^{h}$ (referred to as the height dependent variables) at $10 \%$ intervals from $h=10 \%-90 \%$; the axis lengths of a paraboloid fitted to a tree crown $\left[S_{i}^{g(a)}, S_{i}^{g(b)}\right]$; the percentage of points per height layer of a tree $S_{i}^{d}$ (referred to as the density dependent variables) at 10 intervals from $d=1-10$; and the point count ratios by reflection type (single $S_{i}^{\text {single }}$, first $S_{i}^{n_{1}}$, middle $S_{i}^{n_{2}}$ ) (see Reitberger et al. (2009)). Additionally, for each tree segment $i$, the crown polygon area $A_{i}^{p}$ and the minimum enclosing circle of the projected polygon $\min _{i}^{e c}$ were extracted.

ii) Radiometric features:

The mean intensity of the single $S_{i}^{I_{1}}$ and first $S_{i}^{I_{2}}$ reflections, the intensity $S_{i}^{I_{j}^{H}}$ histograms, and the pulse widths $S_{i}^{P W_{j}^{H}}(j=1, \ldots 10)$ were extracted for each spectral channel within each 3D tree segment $i$ (Reitberger et al., 2009; Yao et al., 2012). Furthermore, the number of spectral bands in the MSLS data offered a unique opportunity to measure the band variances and covariances that were impossible for approaches that use single wavelength ALS data. Six independent band variance and covariance features $S_{i}^{u k}$ (the intensity covariance between channels $u$ and $k(u=1, \ldots, 3 ; k=u, \ldots, 3))$ were obtained that corresponded to the upper triangle of the band covariance matrix.

iii) Bag-of-Words model:

This model is an established method for representing high-level characteristics used for classification purposes (Toldo et al., 2010). The 
main aim of the Bag-of-Words (BoW) model is to approximate the feature vectors using a vector quantization algorithm with a set of prototypes. As proposed by Weinmann et al. (2015a), our BoW model $S_{i}^{B o W \cdot[C]_{b}^{n d}}$ contained eight geometric features generated from the local covariance matrix. In the BoW model, $C$ stands for the linearity, planarity, scattering, omnivariance, anisotropy, eigenentropy, sum of eigen values, and change of curvature that are commonly used in 3D lidar data processing. The frequency histograms for each feature were then constructed using 2-14 bins $b$ that generated a simple BoW model. This was conducted for seven spherical point neighborhood sizes with radii of $n d=0.2-1.6 \mathrm{~m}(0.1)$ that were later used for computing the covariance matrix. The optimal neighborhood radii $n d$ were empirically determined using the tree crown diameters of the study area to limit the generated feature count and prevent overfitting problems.

\subsubsection{Feature selection}

In this study, we used a large number of features to classify the tree species. All the features presented in subsec.5.4.4 sum up to a long list with around 4000 elements. However, only a small number of the features were meaningful and suited for the classification. The high number of features raises the methodical problem that the large hyper-dimensional feature space faces a spare number of samples (Fassnacht et al., 2016). Therefore, a feature selection method needs to be applied that identifies and removes the irrelevant and redundant attributes from the data that do not contribute to the accuracy of the classification model. Guyon and Elisseeff (2003); Liu et al. (2010); Weinmann et al. (2015a) reported on feature selection techniques for finding the most robust subsets of the relevant features to optimize the classification accuracy and to improve the computational efficiency. They are subdivided into wrapper methods, filter methods, and embedded methods.

Here, we proposed a wrapper method referred to as stepwise forward selection (Hastie et al., 2001) that begins with a small feature set randomly selected from the full set and then proceeds in an iterative fashion, selecting one additional feature in each step. A single iteration inspected every available feature by adding it to the active feature set and obtaining an estimate of the classification error rate on the augmented data through cross-validation. The feature that entered the active set with the lowest error rate was incorporated into the result set, and the iterations proceeded. The process was terminated when the inclusion of additional features ceased to decrease the classification error rate. In order to avoid any randomness effects, the selection procedure was repeated 5 times for each scenario (see subsec.5.5.1) and the results were similar for all the iterations. Further, this wrapper method used the multinomial logistic regression of the subsec.5.4.6 as the predictive model. The final result was a list of features organized in ascending order according to the error rate generated in the classification. In this study, the first members on the list were interpreted as the most important features with the highest 
contribution to the classification result.

\subsubsection{Tree Species classification}

To classify tree species based on the features extracted and discussed in subsec.5.4.4, we applied a multinomial logistic regression. Logistic regression models represent the probability distribution of the class label $y$ as follows:

$$
p\left(y_{i}=k \mid x_{i} ; \Theta\right)=\exp \left(\theta_{k}^{T} x_{i}\right) /\left[1+\sum_{l} \exp \left(\theta_{l}^{T} x_{i}\right)\right]
$$

where $x_{i} \in X, i=1, \ldots, M$ denotes the $M$ feature vectors of the training examples and $y_{i}$ represents their corresponding labels. Training the model involves minimizing the penalized joint negative log-likelihood of the training examples with respect to the $\beta>0$ term, as shown in Eq.5.5:

$$
\min _{\Theta} \sum_{i=1}^{M}-\log p\left(y_{i} \mid x_{i} ; \Theta\right)+\beta\|\Theta\|_{1}
$$

where $\|\Theta\|_{1}$ is the regularization term that increases the sparsity of the coefficient vector $\Theta$. After feature selection, a few features remain and the sparsity effect is not very significant. Therefore, a penalized logistic regression can be applied using the following model:

$$
\frac{1}{M} \sum_{i=1}^{M} L(\beta, X, y)-\lambda\left[\frac{(1-\alpha)\|\beta\|_{2}^{2}}{2}+\alpha\|\beta\|_{1}\right]
$$

where $\alpha$ is a balance coefficient between penalties $L_{1}$ (lasso) and $L_{2}$ (ridge), and the optimal value for $\lambda$ can be selected using cross-validation. Eq.5.6 is defined as the 'elastic net penalty', that is a regularized-generalized model and is implemented in the glmnet function as an $R$ source package.

\subsubsection{Evaluation}

The classification accuracy was estimated and evaluated based on the results of the matching step between the reference data and the 3D segmented trees in the upper canopy layer (see subsec.5.3.2) (with a proportion of $60 \%$ $40 \%$ for training and testing the classifier) with respect to the tree species distribution. We used 15 -fold cross-validation to obtain the overall classification accuracy, correctness, and completeness as a compromise between the computational efficiency and reducing the effects of randomness.

Moreover, we evaluated the following combined feature scenarios for the tree species classification: (i) imagery features, (ii) MSLS point cloud features, (iii) combined features (from MSLS data and aerial imagery), (iv) single wavelength (1550 nm (Ch1), $1064 \mathrm{~nm}(C h 2)$, and $532 \mathrm{~nm}(C h 3)$ ) features, and (v) combined features extracted from single wavelength lidar data (1550 nm (Ch1), $1064 \mathrm{~nm}(C h 2)$, and $532 \mathrm{~nm}(C h 3))$ and aerial imagery. 
Table 5.5: Results of tree species classification. Best results are highlighted in bold.

\begin{tabular}{|c|c|c|}
\hline $\begin{array}{l}\text { Scenario } \\
\text { number }\end{array}$ & Feature set & $\begin{array}{l}\text { Average } \\
\text { overall } \\
\text { accuracy } \\
(\%)\end{array}$ \\
\hline 1 & $\begin{array}{l}\text { Multispectral imagery : Channel mean values and covari- } \\
\text { ance matrix }\end{array}$ & 54 \\
\hline 2 & Multispectral imagery : Gabor & 64 \\
\hline 3 & Multispectral imagery : GLCM & 68 \\
\hline 4 & $\begin{array}{l}\text { Multispectral imagery : Channel mean values and covari- } \\
\text { ance matrix + Gabor + GLCM }\end{array}$ & 66 \\
\hline 5 & MSLS point cloud : Geometric & 68 \\
\hline 6 & MSLS point cloud : Geometric + Radiometric & 75 \\
\hline 7 & MSLS point cloud : BoW (0.3-1.6 m) & 54 \\
\hline 8 & $\begin{array}{l}\text { MSLS point cloud : Geometric }+ \text { Radiometric }+ \text { BoW } \\
(0.3-1.6 \mathrm{~m})\end{array}$ & 78 \\
\hline 9 & Combined*: MSLS (Geometric + Radiometric) + GLCM & 75 \\
\hline 10 & $\begin{array}{l}\text { Combined*: MSLS (Geometric + Radiometric + BoW } \\
(0.3-1.6 \mathrm{~m}))+ \text { GLCM }\end{array}$ & 76 \\
\hline 11 & $\begin{array}{l}\text { Combined*: MSLS (Geometric + Radiometric + BoW } \\
(0.3-1.6 \mathrm{~m}))+ \text { GLCM + Gabor }\end{array}$ & 74 \\
\hline 12 & $\begin{array}{l}\text { Single wavelength (channel Ch1): Geometric }+ \text { Ra- } \\
\text { diometric }+\operatorname{BoW}(0.3-1.6 \mathrm{~m})\end{array}$ & 69 \\
\hline 13 & $\begin{array}{l}\text { Single wavelength (channel Ch2) : Geometric }+ \text { Ra- } \\
\text { diometric }+ \text { BoW }(0.3-1.6 \mathrm{~m})\end{array}$ & 71 \\
\hline 14 & $\begin{array}{l}\text { Single wavelength (channel Ch3) : Geometric }+ \text { Ra- } \\
\text { diometric }+\operatorname{BoW}(0.3-1.6 \mathrm{~m})\end{array}$ & 65 \\
\hline 15 & Single wavelength (channel $C h 1)+$ Multispectral imagery & 69 \\
\hline 16 & Single wavelength (channel $C h 2)+$ Multispectral imagery & 72 \\
\hline 17 & Single wavelength (channel $C h 3)+$ Multispectral imagery & 60 \\
\hline
\end{tabular}




\subsection{Results and discussion}

\subsubsection{Main outcomes}

The quantitative evaluation results of the tree species classification for the different feature combinations is presented in Table 5.5. The numbers in Table 5.5 refer to the average classification accuracy, and the best result from each strategy is highlighted. Note that ca. $10 \%$ of the total trees were removed from the further analyses due to the matching strategy and the height filtering (see subsec.5.3.2). In Table 5.5, the initial classification accuracy obtained using only the channel mean values and the covariance matrix for the aerial imagery (near infrared $n i r$, red $r$, and green $g$; scenario 1) could be improved by $14 \%$ if the GLCM features were included (scenario 3 ). However, as shown in scenario 2 , the accuracy was approximately $4 \%$ lower when the Gabor features were used than that with the GLCM features in scenario 3. Overall, the Gabor features did not considerably contribute to the classification accuracy. Further, we conducted scenarios 1 to 4 to demonstrate the sole power of the multispectral aerial imagery and the impact of missing lidar intensity on the tree class classification.

We continued with scenarios 5 to 8 related to the classification accuracy provided by the MSLS point cloud. The results of scenario 6 demonstrated that adding the radiometric features to the geometric features (scenario 5) improved the classification accuracy by about $7 \%$. This was consistent with the recent studies by Heinzel and Koch (2011) and Shi et al. (2018a), who emphasized the importance of the radiometric features from single wavelength ALS data for tree species classification. Among the MSLS point cloud-based features, the BoW model features alone provided the lowest accuracy by $54 \%$ (scenario 7). Comparing the results collected from different feature sets (scenarios 9 to 11) indicated that the overall accuracy was better when using only the MSLS point cloud features (scenario 8) than the classification based on the aerial imagery (scenario 4), or on the combined features from both datasets (scenarios 9 to 11 ).

In this study, the addition of imagery-based features to the MSLS point cloud features did not notably contribute to the tree species identification. It is evident from Table 5.5 that in scenario 9 the same accuracy of $75 \%$ was achieved by adding the GLCM features to the MSLS point cloud features (radiometric and geometric features). Adding the BoW model features (scenario 10) to scenario 9 only increased the accuracy by $1 \%$. However, the Gabor features reduced the accuracy to $74 \%$ (scenario 11). Furthermore, according to scenarios 12,13 , and 14 , accuracies of $69 \%, 71 \%$, and $65 \%$ were estimated for the single spectral channels of $C h 1, C h 2$, and $C h 3$ (when used individually), respectively. These results were clearly worse in performance compared to the combined features (scenarios 9 to 11) and the MSLS point cloud features (scenario 8). Regarding the scenarios focused on the combined features from single wavelength lidar data with aerial imagery, the best result of $72 \%$ was achieved for the combination of the single wavelength channel Ch2 (1064 $\mathrm{nm}$ ) and the aerial imagery features (scenario 15). The results indicated that an overall accuracy of $69 \%$ was achieved for the fused features extracted 
from the single wavelength channel $C h 1(1550 \mathrm{~nm})$ and the multispectral imagery (scenario 16). Furthermore, in the case of the features extracted and combined from the single wavelength channel $C h 3(532 \mathrm{~nm})$ and the aerial imagery, a classification accuracy of $60 \%$ was obtained (scenario 17).

We now focus on the detailed Norway spruce, European beech, Silver fir, and snags mapping. The confusion matrix for the tree classes classification that summarize the correctness and completeness are presented in Tables 5.6, 5.7 and 5.8 based on the scenarios 4,8 and 10 from Table 5.5, respectively. The multispectral imagery features alone (scenario 4) produced the lowest kappa value of 0.57 (Table 5.6), while the combined features (scenario 10), provided a remarkably higher value of 0.64 for the kappa (Table 5.8). The results showed that the features from the multispectral aerial imagery did not provide strong information for the detailed classification in comparison to the features extracted from the MSLS point cloud. Furthermore, in scenarios 8 (Tables 5.7) and 10 (Tables 5.8) for Norway spruce and European beech, very similar correctness and completeness values were achieved. However, in Table 5.7, Silver fir and snags had slightly better accuracies in terms of correctness and completeness compared to the ones in Table 5.8.

Table 5.6: Confusion matrix of classification performance from scenario 4 using features extracted from multispectral imagery.

\begin{tabular}{|c|c|c|c|c|c|c|}
\hline & \multicolumn{6}{|c|}{ Reference } \\
\hline \multirow{7}{*}{ 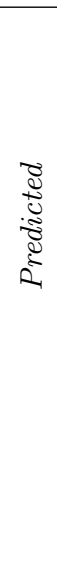 } & & $\begin{array}{l}\text { Norway } \\
\text { spruce }\end{array}$ & $\begin{array}{l}\text { European } \\
\text { beech }\end{array}$ & Silver fir & Snag & $\begin{array}{l}\text { Correctness } \\
(\%)\end{array}$ \\
\hline & $\begin{array}{l}\text { Norway } \\
\text { spruce }\end{array}$ & 63 & 5 & 12 & 1 & 78 \\
\hline & $\begin{array}{l}\text { European } \\
\text { beech }\end{array}$ & 8 & 60 & 6 & 7 & 74 \\
\hline & Silver fir & 10 & 1 & 19 & 6 & 50 \\
\hline & Snag & 1 & 3 & 8 & 30 & 63 \\
\hline & $\begin{array}{l}\text { Completeness } \\
(\%)\end{array}$ & 71 & 67 & 46 & 51 & \\
\hline & $\begin{array}{l}\text { Overall } \\
\text { accuracy: } \\
66 \%\end{array}$ & $\begin{array}{l}\text { Kappa } \\
\text { value: } 0.57\end{array}$ & & & & \\
\hline
\end{tabular}

The highest level of accuracy (scenario 8, kappa value: 0.69, Table 5.7) was obtained for the combination of the radiometric, geometric, and BoW model (0.3-1.6 $\mathrm{m})$ features extracted from the MSLS point cloud. High values of $95 \%$ and $93 \%$ for correctness and completeness, respectively, were achieved for Norway spruce. The correctness and completeness for European beech with $83 \%$ and $86 \%$, respectively, were also fairly good. Silver fir trees that were proportionally less represented in the study area than spruces, were classified with $58 \%$ and $61 \%$ completeness and correctness, respectively. Finally, snags were detected with $73 \%$ completeness and $76 \%$ correctness. The average rate of false negatives for the fir trees and snags in scenario 8 amounted to 
Table 5.7: Confusion matrix of classification performance from scenario 8 using features extracted from MSLS point cloud.

\begin{tabular}{|c|c|c|c|c|c|c|}
\hline & & & Reference & & & \\
\hline & & $\begin{array}{l}\text { Norway } \\
\text { spruce }\end{array}$ & $\begin{array}{l}\text { European } \\
\text { beech }\end{array}$ & Silver fir & Snag & $\begin{array}{l}\text { Correctness } \\
(\%)\end{array}$ \\
\hline & $\begin{array}{l}\text { Norway } \\
\text { spruce }\end{array}$ & 76 & 0 & 0 & 4 & 95 \\
\hline 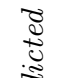 & $\begin{array}{l}\text { European } \\
\text { beech }\end{array}$ & 0 & 69 & 11 & 3 & 83 \\
\hline है & Silver fir & 1 & 7 & 22 & 7 & 61 \\
\hline & Snag & 5 & 3 & 4 & 39 & 76 \\
\hline & $\begin{array}{l}\text { Completeness } \\
(\%)\end{array}$ & 93 & 86 & 58 & 73 & \\
\hline & $\begin{array}{l}\text { Overall } \\
\text { accuracy: } \\
78 \%\end{array}$ & $\begin{array}{l}\text { Kappa } \\
\text { value:0.69 }\end{array}$ & & & & \\
\hline
\end{tabular}

Table 5.8: Confusion matrix of classification performance from scenario 10 using combined features extracted from MSLS point cloud and multispectral imagery.

\begin{tabular}{|c|c|c|c|c|c|c|}
\hline & & & Reference & & & \\
\hline & & $\begin{array}{l}\text { Norway } \\
\text { spruce }\end{array}$ & $\begin{array}{l}\text { European } \\
\text { beech }\end{array}$ & Silver fir & $\overline{\text { Snag }}$ & $\begin{array}{l}\text { Correctness } \\
(\%)\end{array}$ \\
\hline & $\begin{array}{l}\text { Norway } \\
\text { spruce }\end{array}$ & 74 & 0 & 0 & 4 & 94 \\
\hline 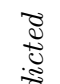 & $\begin{array}{l}\text { European } \\
\text { beech }\end{array}$ & 0 & 69 & 12 & 3 & 83 \\
\hline జे & Silver fir & 2 & 7 & 19 & 8 & 54 \\
\hline & Snag & 5 & 3 & 6 & 36 & 71 \\
\hline & $\begin{array}{l}\text { Completeness } \\
(\%)\end{array}$ & 92 & 86 & 51 & 69 & \\
\hline & $\begin{array}{l}\text { Overall } \\
\text { accuracy: } \\
76 \%\end{array}$ & $\begin{array}{l}\text { Kappa } \\
\text { value:0.64 }\end{array}$ & & & & \\
\hline
\end{tabular}

$39 \%$ and $24 \%$, respectively. These results might be due to the spectral and structural similarities between these tree classes.

\subsubsection{Feature assessment}

In Table 5.9, we focused on analyzing the important features listed for the scenarios 4,8 , and 10 . The scenarios 8 and 10 presented the best classification results and the scenario 4 contained all of the features extracted from the aerial imagery. Note that these scenarios are representative for their different 
Table 5.9: List of the most important features under different classification scenarios (each scenario is defined in Table 5.5). Each scenario has 30 features selected via the feature selection step. The below mentioned feature abbreviations are explained in subsec.5.4.4.

\begin{tabular}{ll}
\hline Scenarios & Top five features \\
\hline Scenario 4: Imagery features & $\begin{array}{l}G L C M .\left[\text { contrast }_{8}^{\mu}, G L C M \cdot[\text { correlation }]_{8}^{\sigma},\right. \\
\text { mean }_{n i r}, \text { Cov }_{r r}, G L C M .[\text { energy }]_{8}^{\mu}\end{array}$ \\
\hline $\begin{array}{l}\text { Scenario 8: MSLS point cloud fea- } \\
\text { tures }\end{array}$ & $S^{I_{6}^{H}}(C h 2), S^{I_{1}}(C h 2), S^{21}, S_{1.3}^{g}$, \\
\hline & $S^{\text {BoW. [scattering }]_{7}^{0.3-0.8}}$ \\
\hline $\begin{array}{l}\text { Scenario 10: Combined* features } \\
\text { * Features extracted from MSLS point cloud } \\
\text { and aerial imagery are combined. The nir } \\
\text { and } r \text { terms stand for the near infrared and } \\
\text { red bands in the aerial imagery, respectively. }\end{array}$ & $S^{I_{6}^{H}}(C h 2), S^{I_{1}}(C h 2), S^{I_{1}}(C h 1), S^{21}$, min $_{e c}$ \\
\end{tabular}

dataset combinations (multispectral aerial imagery, MSLS point cloud, combined aerial imagery and MSLS point cloud). After the feature selection step, from 4000 features identified, 30 features were selected as the most important features for the classification. The classification based on imagery features (scenario 4) was mainly supported by the GLCM features GLCM.[contrast $]_{8}^{\mu}$, GLCM.[correlation $]_{8}^{\sigma}$, the mean of the near infrared nir channel, the variance of the red $r$ channel $C_{\text {ov }}$, and GLCM. [energy $]_{8}^{\mu}$. Apparently, the contrast represented by $G L C M$. $[\text { contrast }]_{8}^{\mu}$ was the best discriminative feature in this scenario mainly because of the star-shaped texture of the coniferous trees versus the nearly smooth texture of the broad-leaf trees. The top GLCMbased features represented by the GLCM.[contrast $]_{8}^{\mu}, G L C M .[\text { correlation }]_{8}^{\sigma}$, and $G L C M$. [energy $]_{8}^{\mu}$ clearly indicated that texture features measured from the images were helpful for discriminating between the four tree classes. Furthermore, these meaningful GLCM-based features were followed by features describing the near infrared nir and the variance of the red $r$ channels. This was expected because the red edge is best suited for vegetation classification (Schuster et al., 2012). In particular, the snags showed a specific spectral signature in the near infrared nir channel.

In this section we focus on scenarios 8 and 10 that represented the best MSLS point cloud cases and the combination of all features, respectively. The intensity histogram features $S^{I_{6}^{H}}$ that were provided by channel $C h 2$ exhibited the highest contribution to the species classification. Interestingly, the geometric features, such as the percentile of points per height layer of a tree $S_{i}^{h}$ describing the laser beam penetrations in the segmented 3D tree clusters had less impact compared to the intensity-based features on the classification results. This is due to the fact that these features were not present in the final top five features list for the classification. Moreover, the mean intensity of the single channel $C h 2$ reflection $S^{I_{1}}(C h 2)$ has performed better than those from channels $C h 1$ and $C h 3$. This result was consistent 
with the findings of previous studies, such as Yu et al. (2017) and Axelsson et al. (2018) who emphasized the importance of the features extracted from channel $C h 2$. Note that the classification based on the MSLS point cloud was $10 \%$ better than the classification with aerial imagery (see Table 5.5). Finally, when we analyzed the top five features for the combined features (scenario 10) we noticed that the top three features $S^{I_{6}^{H}}(C h 2), S^{I_{1}}(C h 2)$, and $S^{I_{1}}(C h 1)$ were provided by the MSLS point cloud. This in turn explained why the classification with the extracted features from the MSLS data (best case $78 \%$ - scenario 8) was nearly identical to the classification using the combined features (best case 76\% - scenario 10) (see also Table 5.5). Interestingly, the minimum enclosing circle of the crown polygon $\min _{e c}$ also supported these scenarios. Apparently, the single tree crown sizes were a discriminative feature in this scenario, mainly due to the different crown diameters between the coniferous and broad-leaf trees.

Finally, we address the learning curves that the feature selection process provided for each scenario. The learning curves in Fig.5.6a,b showed that approximately 15 features for the BoW model and 20 features for the other sets played a remarkable role in improving the accuracy. Beyond this point, the inclusion of more features did not improve the classification accuracy. In Fig.5.6a,b, the classification accuracy of the combined geometric and radiometric features (scenario 6, Table 5.5) was better than that of the BoW features (three neighborhood sizes). However, the BoW features appeared to contain additional information because their inclusion increased the final accuracy from $75 \%$ to $78 \%$ (see Table 5.5, scenarios 6 and 8). If we compared the three BoW models in Fig5.6a,b, the $0.2 \mathrm{~m} S_{i}^{B o W^{0.2}}$ point neighborhood was the least informative. The best results were obtained for neighborhood sizes of $0.3-0.8 \mathrm{~m} S_{i}^{B o W^{0.3-0.8}}$. Further, the feature selection performance of the spectral channel $C h 2(1064 \mathrm{~nm})$ is presented in Fig.5.6b. The best performance was achieved for the combined geometric and radiometric features (scenario 13, Table 5.5). Moreover, among the aerial imagery scenarios, the lowest classification error was achieved using the GLCM features (scenario $3)$.

The important feature selection step was applied to extract robust feature subsets that optimized the classification accuracy. It is important to note that the accuracy was on average reduced by nearly $6 \%$ when all the generated features (without feature selection step) were used. Recent feature selection approaches primarily applied wrapper methods, filter methods, or embedded methods (Guyon and Elisseeff, 2003). In general, the wrapper methods (such as forward feature selection) performed better than the other mentioned techniques in terms of classification accuracy (Sikora and Piramuthu, 2007). However, our feature selection method required as a wrapper method high computational effort because the classifier model needed retraining as part of the cross-validation used for accuracy assessment purposes. 


\section{Feature selection performance}

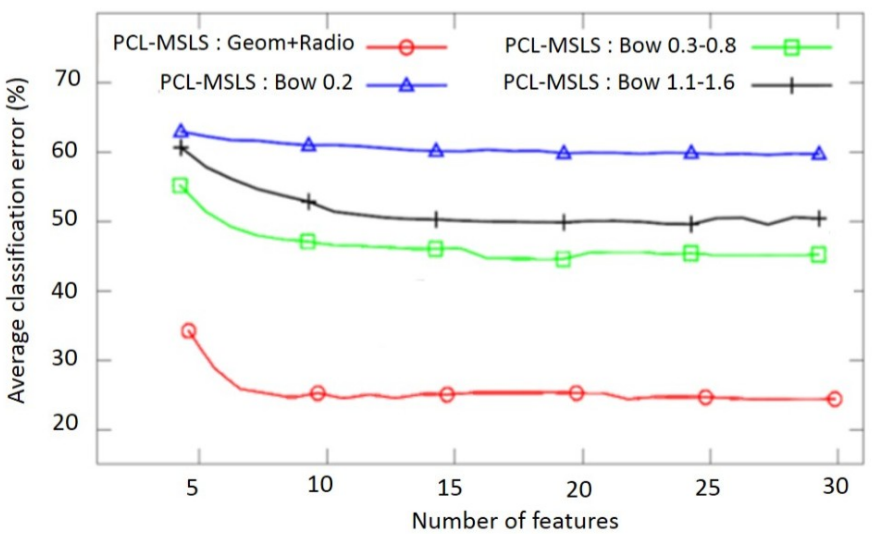

(a)

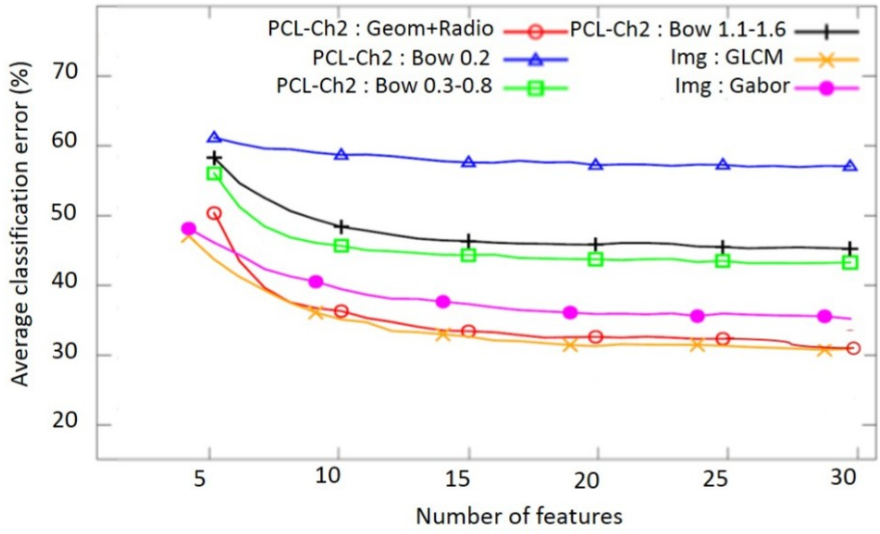

(b)

Figure 5.6: Learning curves describing the average error as a function of feature count; $P C L-M S L S$ refers to the MSLS point cloud, Img is the aerial imagery, $P C L-C h 2$ refers to single wavelength channel $C h 2$, and $B o W$ refers to the BoW model with various neighborhood sizes. The curves correspond to the classification performance in terms of average error (\%) after feature selection step using features extracted from a) the MSLS point cloud and b) the aerial imagery and single wavelength channel $C h 2$.

\subsubsection{Comparison with related work}

The results of this study should be compared to those of previous studies (Shi et al., 2018a,b) conducted on the Bavarian Forest National Park that classified the six tree species (Spruce, Beech, Fir, Maple, Rowan, and Birch). The accuracy of our study was $20 \%$ higher under leaf-on conditions than the findings of Shi et al. (2018a), who used only features extracted from single 
wavelength ALS data for multiple tree species classification. However, Shi et al. (2018b) recently showed a higher accuracy of $85 \%$ (5\% improvement) by combining the hyperspectral imagery and single wavelength ALS data features. This was mainly related to the notable contribution of the functional plant traits (equivalent water thickness, leaf mass per area and leaf chlorophyll) and spectral features from the hyperspectral data.

Our results demonstrated that the MSLS point cloud features increased the accuracy of the classification of four tree classes by approximately 7 to $13 \%$ compared to the single wavelength features. Moreover, combining the features from the aerial imagery and MSLS data did not greatly enhance the results compared to only applying features from multispectral lidar data. Our study verified the findings of $\mathrm{Yu}$ et al. (2017) (overall accuracy: 85.9\%, kappa: 0.75 ) and Axelsson et al. (2018) (overall accuracy: $75.5 \%$ ), who stated that intensity-related features extracted from MSLS data, specifically those from channel $C h 2$, were the most important for classifying tree species. We found that in both scenarios 8 and 10 (see Table 5.9) the intensity histogram-based feature $S^{I_{6}^{H}}(C h 2)$ played a more important role in the species identification than any other radiometric or geometric feature. As far as the classification using single wavelengths was concerned, our findings confirmed the results of Shi et al. (2018a) who concluded the importance of the intensity-related features (within the top seven features) when employing an embedded feature ranking strategy for the Random Forest classifier. Moreover, Budei et al. (2018) showed that the intensity-based features of infrared and green channels of a three-wavelength ALS system using the Random Forest classifier can improve the detailed species identification accuracy in a temperate forest compared to single channel ALS systems. Concluding, our analysis was based on sample plots with relatively limited variability between the tree species. Therefore, the applicability of our identified important features needs to be tested further in other study sites.

Finally, we compare the snag classification with other approaches obtained from the same forest site. Yao et al. (2012) tackled for the first time snag detection using only the lidar intensity (single wavelength $1550 \mathrm{~nm}$ ) and geometric features such as the crown shape and point height distribution. The study reported a classification accuracy between $71 \%$ to $73 \%$. Recently, Polewski (2017) presented a method that uses single tree 3D segments along with multispectral aerial imagery. Based on features generated from the covariance matrix of the three image channels, a two-class classification (snag and non-snag) led to an accuracy of ca. $88 \%$ that was remarkably better than our results. However, the study of Polewski (2017) used only reference data generated by visual inspection of the tree point cloud and used orthophotos superimposed with the crown polygons.

In the end, we show in Fig.5.7 a 3D point cloud representing a forest scene with three tree species and snag classification. 


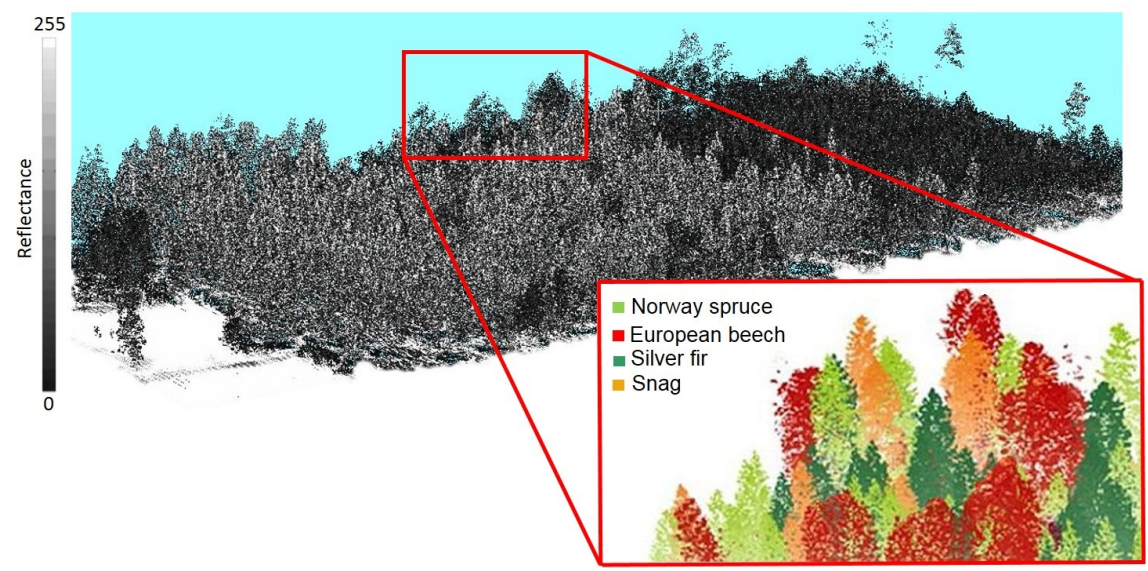

Figure 5.7: Classified individual tree classes for a sample area in Bavarian Forest National Park (spectral wavelength of $1064 \mathrm{~nm}$ ).

\subsection{Conclusions}

In summary, our experiment showed that MSLS point clouds can aid in the characterization of tree species and snags in approaches that work at the single tree level. The use of features extracted from the MSLS point cloud selected using the feature selection step, mainly the intensity-related features from spectral channel $C h 2(1064 \mathrm{~nm})$, notably improved the classification rate, achieving an overall accuracy of $78 \%$. Moreover, the classification accuracy did not improve when multispectral imagery were added to the MSLS data. If no lidar-based features (geometry and intensity) were used, the combination of features extracted from the multispectral aerial imagery led to a weak classification accuracy of $66 \%$.

This study performed one of the first experiments examining the applicability of MSLS data for tree species classification on a temperate forest. Instead of using one single instrument, we simulated MSLS data by combining sensor data acquired from two different instruments on the same day with stable weather conditions. The flying height and the atmospheric attenuation was the same for the two sensors and almost the same for the third one. Of course, this system configuration has different scan angles and different sensors as well. All in all, we believe that this setup provided radiometric data comparable to those captured by a single instrument consisting of three non-collinear lidar units, e.g. the Titan sensor from Optech. Clearly, the use of a single instrument has advantages over multiple sensors in terms of data processing. However, both instrument approaches have the drawback that non-collinear laser beams are used meaning that the backscattered pulses do not necessarily result from the same part of the object.

Furthermore, as expected, the feature selection step considerably reduced the high dimensional feature space used to optimize the classification accuracy. Based on the prominent features list, the feature selection procedure was 
able to identify the most discriminative features. Moreover, the classification deteriorated by about $6 \%$ if all the extracted features without the feature selection step had been used.

From a practical point of view, the accuracy level is not yet optimal (up to $90 \%$ ). Apparently, the radiometric information from the non-collinear MSLS data provided at three distinct wavelengths limited the classification of the tree species and snags to this accuracy level in a temperate forest. Further research needs to be conducted to investigate the relationships between the structural tree crown characteristics and the MSLS point cloud-based features to determine their impact on the classification accuracies for multiple tree species. Furthermore, a collinear multispectral lidar system (whose emitted laser beams strike the same target simultaneously) or the fusion of lidar with hyperspectral imagery may improve the detailed tree species classification.

\subsection{Acknowledgment}

We would like to thank Riegl company for providing us the excellent lidar data and the equation to convert amplitude to reflectance values. 
Synthesis

6 


\subsection{Introduction}

Forest structure analysis can describe various ecosystem services, help to understand the underlying canopy processes, and permit projection of future climate conditions. Therefore, accurate estimation of forest structure variables is essential in order to fully understand changes in the forest ecosystem. In the temperate or boreal forests of Europe, the majority of forest structural variables are directly or indirectly measured by standard forest inventories. However, these traditional methods are time-consuming and expensive (Hyyppä et al., 2000). Therefore, an efficient approach for measuring the forest structural attributes is required to ensure sustainable forest management at different scales.

As is mentioned in Chapter 1, forest structure analysis has a fundamental role in forest management, particularly for the authorities that use methods operating at single tree level. Although various methods for forest structure analysis have been investigated and are well established within some of the Scandinavian countries, this still requires detailed exploration in the central European region. Therefore, to develop and promote the current stage, effective methods combined with available data sources are required.

Remote sensing-based methods, and particularly airborne lidar, have shown powerful potential for supporting forest inventories during the last decades (White et al., 2013). Forest structural variables such as tree height, DBH, and mean tree diameter have been widely studied and measured using lidar data across temperate and boreal forest areas. With respect to these facts, this thesis made attempts to reach the primary aim of providing operational methods for lidar-based analysis of forest structural variables.

The final chapter evaluates the most important conclusions of the work carried out as the basis for this thesis. The four selected forest structure variables, namely regeneration coverage, tree stem count per hectare, tree segmentation, and tree species classification using lidar were studied and quantified. Moreover, for further research it is necessary to propose new directions based on the limitations associated with the presented methods. The main findings are categorized according to their relationships to the general research objectives.

\subsection{Estimation of regeneration coverage in a temperate forest by 3D segmentation}

Estimation of regeneration coverage using ALS data have been the focus in earlier studies regarding the important role of the understory canopy layer in future forest planning. The importance of the understory canopy layer lies mainly in the changes in it that happen due to various factors such as tree harvest (Nakamura et al., 1996), forest fire (Bataineh et al., 2006), and insect outbreaks and infestations (McMillin and Allen, 2003). Therefore, the understory layer's status and dynamics turn out to be major indicators of forest health (Kerns and Ohmann, 2004). For instance, Fig.6.1 shows natural 


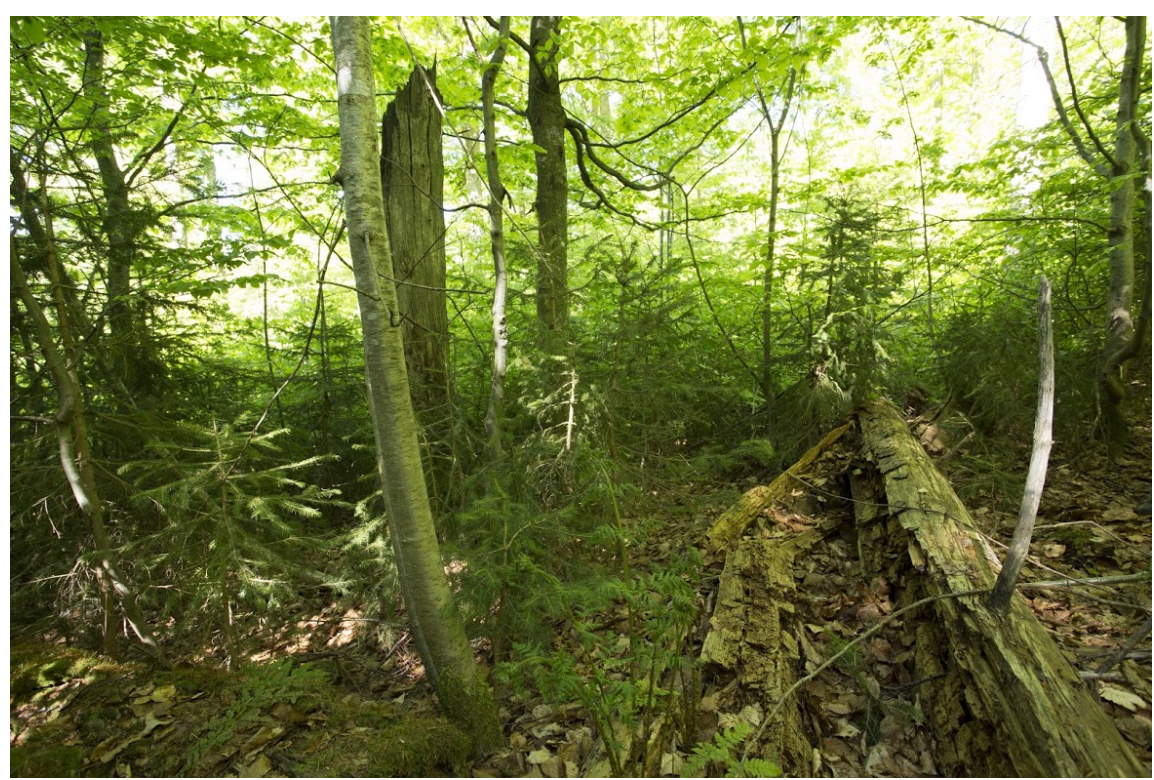

Figure 6.1: An example forest scene with regeneration in the Bavarian Forest National Park.

forest development and regeneration growth after windfalls during 1983 and 1990 in the Bavarian Forest National Park.

Remote sensing methods for 3D analysis of the forest understory have been mostly developed via lidar data that penetrates through the overstory layer and reaches the lower layers. However, there is still limited experience in applying methods to estimate regeneration coverage in multi-layered forests due to the presence of ground vegetation and overlapping crowns in the overstory (Amiri et al., 2016).

The first objective, the structural variable of understory canopy coverage is addressed here. One of the main aims was to propose a framework for the reconstruction of regeneration patches within a sparse lidar 3D point cloud. This was achieved by using the enhanced 3D segmentation algorithm (mean shift clustering combined with Normalized Cut (Yao et al., 2013)), starting from the single point level to construct the 3D regeneration objects through clustering, merging, and filtering steps. The experimental results show that under a low or moderate overstory canopy layer, the proposed method is able to estimate the regeneration coverage with high reliability compared to ground truth data (overall accuracy up to $70 \%$ ).

From a forestry perspective, the extraction of information on regeneration as part of the understory canopy layer is as important as information on any of the overstory layer elements, especially in natural forest regions. Regarding the findings of Amiri et al. (2016), full waveform lidar provides sufficient 
information for the regeneration coverage estimation, due to the high penetration rate of the laser beam through the overstory trees. The study focus in the framework of this thesis was on the estimation of regeneration coverage at the understory canopy layer, which is spatially restricted in protected temperate forest areas with high structural heterogeneity. The encouraging outcomes mainly suggest the possibility of reproducing these experimental regeneration coverage results at practical scales using full waveform lidar data (20-30 points $\left./ \mathrm{m}^{2}\right)$, which is normally available from the managed and nonmanaged forest administrative units in central Europe. Moreover, Fig.6.2a clearly shows the effect and role of the high density overstory canopy layer on the presence or absence of regeneration. Canopy gaps due to natural hazards, cut off regions, or dead trees, as seen in Fig.6.2b, can increase the chance of regeneration growth. The discrete ALS sensors can only record the first and the last laser return pulses and therefore the beam is often not able to represent precisely the details of the intermediate and understory canopy layers. Therefore, 3D point clouds acquired with full waveform ALS scanners can provide an accurate estimation of the regeneration coverage.

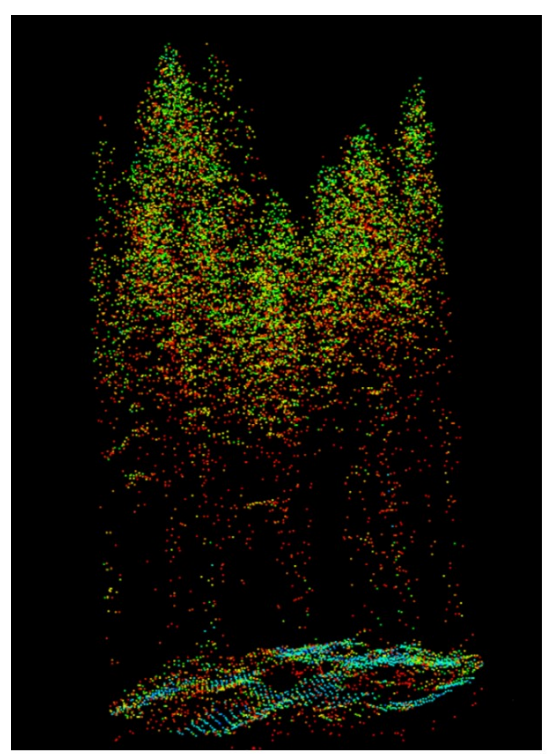

(a)

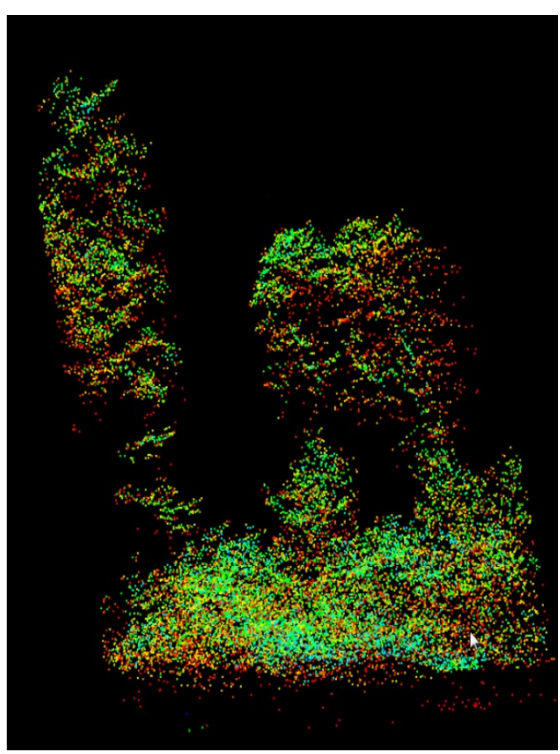

(b)

Figure 6.2: 3D point cloud (30 point $/ \mathrm{m}^{2}$ ) visualization of two sample circular plots with a) absence and b) presence of the regeneration (point clouds are colored by height over DTM). 


\subsection{Detection of single tree stems in forested areas from high density ALS point clouds}

Regarding the detection of single tree stems using ALS point clouds, there has been a relatively low number of contributions that focused on the extraction of positions of individual tree stems. However, the use of TLS data for assessing detailed wood and leaf separation is quite well established (Liang and Hyyppä, 2013; Pfeifer and Winterhalder, 2004). Up to now, different studies that focused on attempts to automatically detect and reconstruct individual tree stems from TLS point clouds have been published. However, this thesis does not consider them because its main focus is on high density ALS data for detection of single tree stems.

The second objective of this thesis was to develop an efficient classifierbased method at point, segment, and object levels for reconstructing lines representing single tree stems from a dense $3 \mathrm{D}$ point cloud. The common flight height of ALS applications usually results in point densities up to 30 points $/ \mathrm{m}^{2}$. However, decreasing the flight height to below $150 \mathrm{~m}$ leads to more laser reflections from tree stems and significantly increases point density. So far, there has been limited persistence regarding the stem detection of single trees using high point density ALS data.

The main goal of the this objective was to detect linear structures in the $3 \mathrm{D}$ point cloud that are likely to represent single tree stems. The proposed method was adapted from the study by Polewski et al. (2015b), which was designed for fallen tree segmentation. It uses a line fitting and optimization approach in the point cloud to determine the lines representing single tree stems. In the first step, the likelihood of points belonging to a tree stem is estimated and the segments containing the highest probability of belonging to the class of stem points are detected. In the next step, the segments are merged through hierarchical clustering to obtain single tree stems. The segment refers to the grouping of points within a fixed length cylindrical neighborhood, which are likely to represent parts of a tree stem. Objects refer to entire tree stems that are composed of groups of similarly aligned segments.

This method indicates that high point density ALS data has great potential for accurately assessing forest structural variables. As seen in Fig.6.3 in the high point density lidar data, there are a relatively higher number of reflections from the stems compared to those in the standard ALS data (up to 30 points $/ \mathrm{m}^{2}$ ). This renders possible the line fitting approach to locate tree stems. As mentioned earlier, the general capacity of high point density lidar data compared to common and low resolution lidar data for practical analysis of forest structural variables is not yet fully explored. From a practical point of view, high point density ALS data is highly recommended, because the stems are poorly visible in the sparse 3D point cloud. However, even with a dense point cloud the proposed method achieves relatively lower accuracy in terms of correctness and completeness for broad-leaf trees than for coniferous trees (Dersch, 2018). The stem position detection indicates that the work flow has some limitations regarding small and very densely packed trees, especially 
in the mixed species plots where the tree crown density is high. Therefore the method requires further investigation.

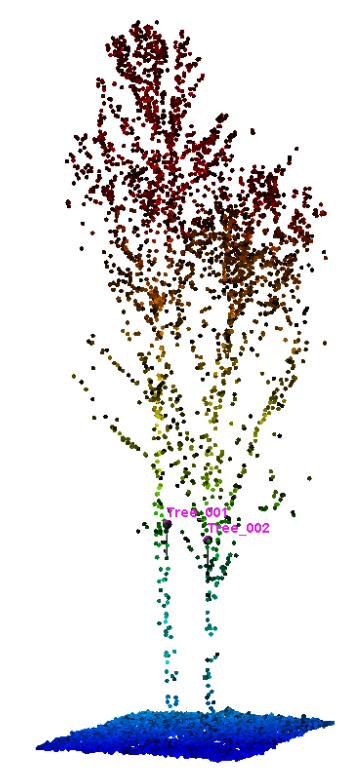

Figure 6.3: 3D visualization of single trees in the high density ALS data with visible stems (point cloud is colored by height over DTM).

Furthermore, the results of the introduced stem detection method can be later used in segmentation algorithms to improve the reliability of the top trees detected through use of the local maxima. The preliminary test on high point density ALS data shows the potential to detect and delineate single trees from bottom to top more accurately (see master thesis of Dersch (2018)). Fig.6.4 shows the preliminary results on broad-leaf tree delineation based on the Normalized Cut algorithm with and without inclusion of stem positions. The extracted stem positions in the sample study areas were combined with the tree tops detected via local maxima. The final accuracy of the Normalized Cut segmentation was slightly improved by merging the local maxima and tree stem positions generated by the stem detection step. The quantitative results in broad-leaf and mixed tree species areas show that the correctness and completeness values were improved. Specifically, the detected stem lines enriched the segmentation quality of the broad-leaf trees. Although, in the cases of very close trees and dense understory, the classifiers and feature sets used at the point and segment levels require further development. Furthermore, the single-tree segmentation work-flow still has difficulty partitioning tree clusters precisely. This could be due to the static value of the Normalized Cut threshold, which needs to be replaced by an adaptive and dynamic approach (Amiri et al., 2018b). 


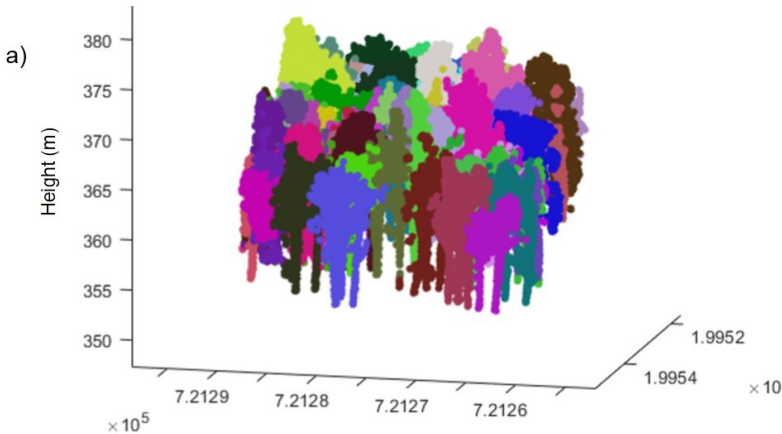

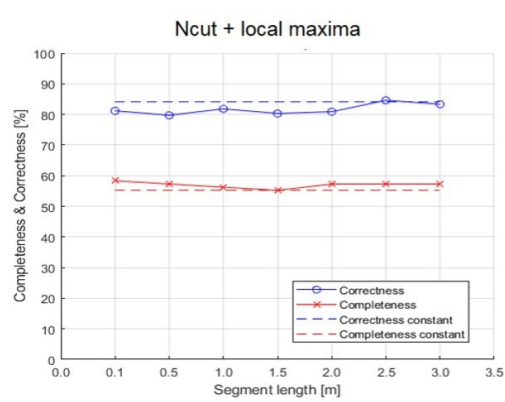

b)

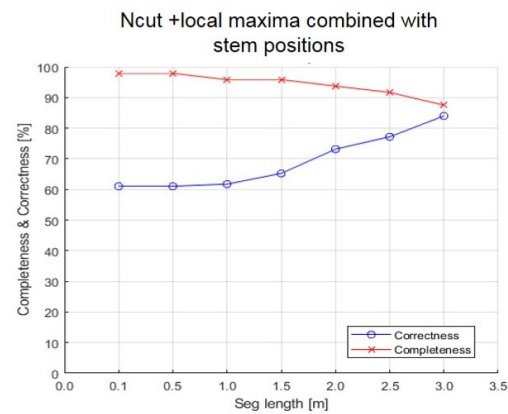

c)

Figure 6.4: a) Visualization of single tree segmentation on a sample area dominated by broad-leaf trees. Correctness and completeness results on the sample plot based on the b) local maxima and c) stem positions combined with local maxima (Dersch, 2018).

\subsection{Adaptive stopping criterion for top-down segmentation of ALS point clouds}

There are multiple lidar-based methods developed for single tree delineation directly from 3D point clouds or from processed CHM. Straightforward methods such as watershed segmentation applied on CHM extracted from lidar achieved up to $45 \%$ delineation accuracy at the overstory level (Heurich, 2008). More sophisticated top-down segmentation approaches such as Normalized Cut applied to waveforms have been reported to deliver more accurate results, especially on the understory level, by taking into account all the information within the 3D data (Reitberger et al., 2009; Yao et al., 2014). However, overlapping crowns and different geometric shapes of nearby trees cause over/under-segmentation in the final results. In order to assess the applicability and generality for forest inventory purposes, these methods still need to be investigated. Therefore, the third objective of the thesis was focused on single tree segmentation in order to reduce the effects of 
over/under-segmentation on the overstory layer by defining an adaptive criterion for stopping or continuing the partitioning process.

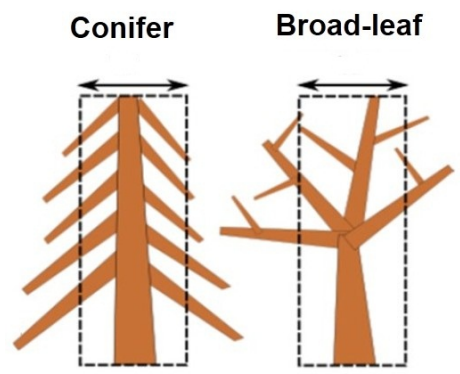

Figure 6.5: Example depiction of geometry shapes of coniferous and broad-leaf trees.

In this context, the main goal was to propose a classifier-based framework in order to improve the segmentation accuracy based on the visual appearance of trees within the 3D point clouds (Fig.6.5). The static scheme of the control parameters in the segmentation algorithm can be replaced by an adaptive scenario applied on the decision level, in order to have flexible tree crown delineation. This was achieved by introducing an adaptive stopping criterion based on the modeling of the coniferous tree crowns with elliptic paraboloids to infer whether a given $3 \mathrm{D}$ scene contains only one or multiple trees. The method was carried out for the coniferous trees in particular, due to the welldefined geometry of their shapes. However, further investigation is required of broad-leaf trees as well, because they appear to be one of the main reasons behind the segmentation errors. In the first step, candidate tree peaks were extracted using the local maxima approach. Then, paraboloid surfaces were fitted at the peaks using a random sample consensus procedure and classified by their geometric properties. At the decision stage to stop or continue the partitioning, a set of non-overlapping paraboloids was used. The results of the classifier learning in the adaptive segmentation algorithm, indicate that the fitting paraboloid function and stopping criterion can both operate quite well across such as the test plots.

The proposed adaptive segmentation method was not fully successful in the partitioning process. In the case of clusters containing more than one single tree but less than two trees, the classifier was not able to provide detailed information for the decision level in the Normalized Cut algorithm (see Fig.6.6). In addition, in the case of broad-leaf trees, the proposed method was not successful at providing satisfactory results. Although the control parameters for the adaptive segmentation can be estimated by a grid search method and empirically for a localized forest area, their transferability to larger scenes could face difficulties. 


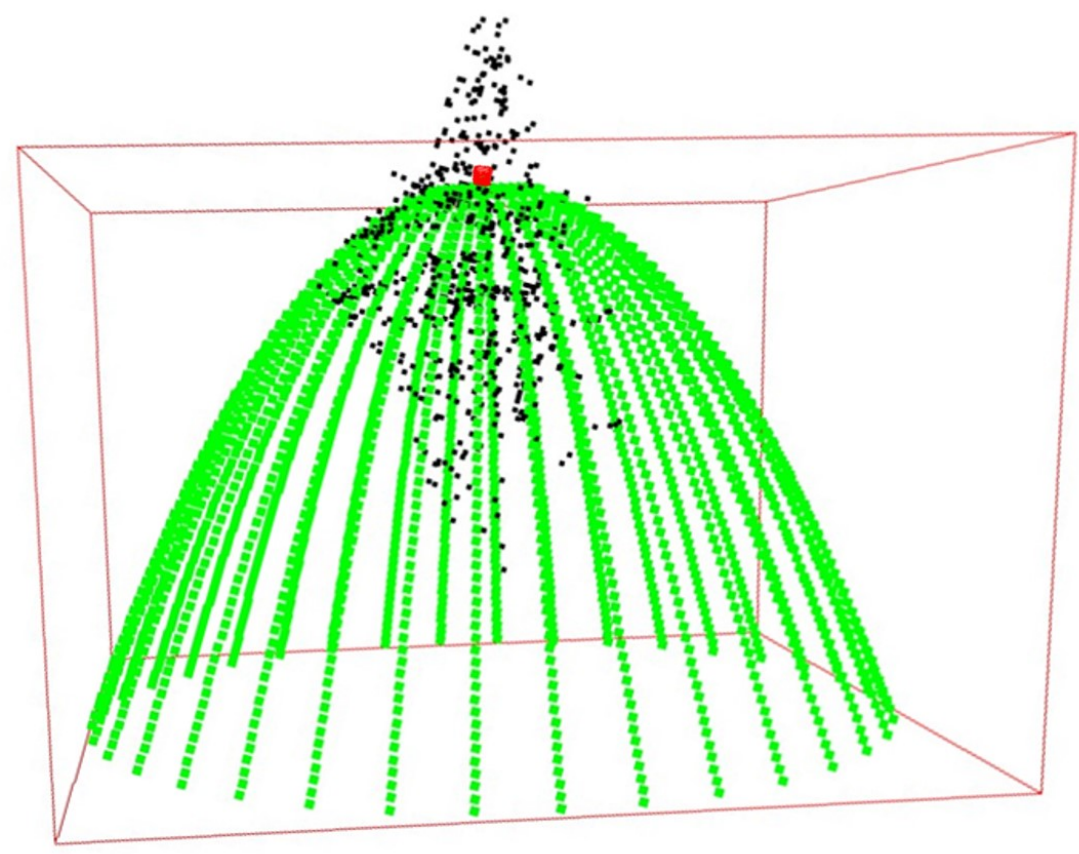

Figure 6.6: An example coniferous tree cluster in a point cloud where the adaptive segmentation and paraboloid fitting was not fully successful in decision level to continue partitioning (the red cylinder shows the detected local maximum for the cluster).

To improve the decision level for the cases mentioned above, alpha shapes and other basic shape descriptor methods were implemented, but the outcomes did not show any significant improvement. Aside from these points, the low point density at the intermediate layer, as well as the non-reliably detected local maxima increased the difficulty of the delineation task. For a practical application, higher point density lidar data combined with the stem detection method proposed in Chapter 3 using 3D shape descriptors is recommended. The idea can be implemented to deal with the mentioned limitations regarding over/under-segmented clusters, accuracy of broad-leaf tree segments and reliability of the local maxima. The detected stem positions can be used in the adaptive segmentation to improve the segmentation accuracy. The tree tops detected by the local maxima can be replaced or improved by the stem positions, especially in the case of broad-leaf trees where local maxima contain false negatives.

At the current stage, there is a necessity to acquire higher point density lidar data. As already shown, standard ALS data cannot contribute to the tree segmentation accuracy and cannot later be classified, particularly in the case of temperate forests with complex overlapping crowns. It is worth mentioning that the geometrical shape of different tree species can change over time due to different factors such as the effect of neighboring trees. Therefore most of 
the shape-based classifiers in temperate forests are not yet fully successful for finding and classifying tree species exactly.

\subsection{Tree species classification by fusing multispectral lidar and aerial imagery}

Previous studies of tree species classification by the application of only geometric features extracted from lidar data show promising results and can be considered the basis for further practical application in temperate central Europe forests. However, it is necessary to note the basic inefficiency of geometrical features for providing detailed information on detailed tree species, which keeps the mentioned approach still dependent on spectral information, field data, and additional 3D features (Hovi et al., 2016; Ørka et al., 2009). A study by Fassnacht et al. (2016) offers a wide range of analysis, a review of remote sensing-based methods, and data for the purpose of tree species classification (for further reading). While the majority of promising results based on lidar data for tree species identification have been provided for the Nordic boreal zone in Europe, the practicality of approaches based on the combination of lidar and spectral (optical imagery) data have been frequently approved in the studies carried out within the temperate zone. As an example, Jakubowski et al. (2013) used a height threshold to separate non-canopy from canopy elements followed by a species classification step based on optical information.

Regardless of the methods applied, there is a high number of tree species studies on combining the features extracted from optical (mainly hyperspectral imagery) and lidar data (Fassnacht et al., 2016). Recently, Shi et al. (2018b) achieved a relatively high accuracy of $85 \%$ using the combined features from hyperspectral imagery and single wavelength ALS data for the classification of six tree species in the Bavarian Forest National Park. It is worth mentioning the that high accuracy was mainly related to the significant contribution of functional plant traits and their spectral features from hyperspectral data. Although the availability of lidar data is useful for deriving information on all the vertical canopy layers, the conducted analysis proved that the information on the forest species composition is similarly important. Therefore, it is suggested that the spectral information be extracted from commonly-available aerial imagery across the temperate European forests and used together with the complementary information in lidar data (Latifi, 2011). As described above, combining spectral and spatial properties of optical imagery and lidar data has gotten great attention for enhancing the existing results for forest structure analysis using single sensor data for tree species classification. However, despite the reported achievements, one of the main limitations in most single sensor-based (lidar) tree species classification methods in temperate forests occurs due to the lack of reasonable spectral signatures of the different tree species (Leckie et al., 2003; Lim et al., 2003; Ghosh et al., 2014).

The fourth objective was focused on tree species classification using multispectral lidar data and aerial imagery. The recently developed multispectral 
lidar techniques are attractive options for forest studies, because not only can they provide 3D structural information, but they can also add spectral information in three different wavelengths (532, 1064, and $1550 \mathrm{~nm}$ ) (StOnge and Budei, 2015; Yu et al., 2017; Axelsson et al., 2018; Amiri et al., 2018a). The intention was to explore the potential of the features extracted from multispectral lidar and aerial imagery for detailed tree species mapping in temperate forests. An improvement of $7-13 \%$ over single wavelength lidar approaches was gained when multispectral lidar data was used. The main results for the individual multispectral point cloud and aerial imagery datasets show that cross-validated (15-fold) accuracies of $78 \%$ and $66 \%$ were achieved, respectively. Moreover, the contribution of the radiometric features of the multispectral lidar data to the classification accuracy was higher than that of geometric features by about $10 \%$. However, the main limitation of the currently available multispectral lidar sensors is the non-collinearity of the laser beams. Additionally, the spectral features extracted from multispectral imagery are included in the pool of features. The selection of the most significant features has to be done carefully: all of the features extracted by a step-wise feature selection step are evaluated in order to select the most significant features for the multiple tree species classification. Recent studies showed that the spectral features extracted from $1064 \mathrm{~nm}$ wavelength data provide the most promising results for the multiple tree species classification (Yu et al., 2017; Axelsson et al., 2018; Amiri et al., 2018a).

Although the usage of the multispectral lidar data for tree species classification is still limited, the above mentioned studies show that multispectral lidar holds the potential to provide detailed information about tree species as long as the single sensor approach is concerned. Furthermore, advances in sensor technology either in multispectral lidar or in fusion of hyperspectral and lidar systems may also improve detailed classification of tree species.

\subsection{Implications of lidar for forest structure analysis in ecological studies}

Research during the past decade has focused on the applicability of lidar for forest inventory and biodiversity. Due to the ability to determine 3D surface coverage using laser scanning sensors over forests, new possibilities have arisen to investigate significant habitat factors. Forest soil analysis, which has a major role in ecosystem diversity, can be conducted using lidar under leaf-off conditions. In addition, it is important to have accurate information about the occurrence, frequency, and reproductivity of different tree species, which can in return help to understand and monitor biodiversity. This would determine how structure variables at tree level would impact the biophysical process of forest ecosystems. Furthermore, the approaches using lidar data can result in better habitat quality maps as a basis for forest biodiversity and management purposes.

Laser scanning has become a comprehensive tool to control and monitor biodiversity of forest areas more sustainably, compared to conventional meth- 
ods. Lidar data is highly accurate and opens up new opportunities for forest inventory and research application fields at single tree level. By using lidar it is possible to have comprehensive coverage of forest dynamics to better understand and describe forests. The estimation of forest structural variables covers different purposes such as habitat modeling, conservation monitoring and habitat suitability for different species.

The research conducted through the framework of this thesis on estimating forest structural variables greatly supports the previously reported gains from using 3D lidar data for single tree-based approaches. In this thesis, methods have been improved for regeneration coverage estimation using 3D segmentation, stem detection using classifier training, individual tree segmentation via an adaptive stopping criterion, and feature-based tree species classification. The method developments contribute valuable tools for forest inventory and biodiversity monitoring in terms of providing more generalized and certain statements on the role of influential factors for modeling forest habitats. The performance and quality of the methods for practical inventories has been investigated and achieved greater accuracy than did the conventional inventory methods.

In this respect, in Chapter 2, the regeneration coverage estimation using full waveform lidar data was investigated and proved that by using the 3D segmentation strategy, the detection rate of regeneration increases. The outcomes reveal that there is a number of other parameters such as the overstory canopy density and species composition that influence the applicability of the proposed method. Chapter 3 is about the detection of single tree stems using a novel classifier-based and line-fitting method and demonstrates that by having high point density ALS data, the tree positions are detected with high accuracy. Chapter 4 is focused on using elliptic paraboloids to deal with over/under-segmentation problems at overstory canopy layers and training a classifier to participate in the decision making level of the Normalized Cut segmentation. This approach significantly improves the Normalized Cut segmentation because the static stopping criterion based on a constant Normalized Cut threshold has been replaced by an adaptive procedure. The adaptive procedure is based on the paraboloid appearance of the tree crowns within the 3D point cloud, and is independent of any internal features of the underlying Normalized Cut segmentation algorithm. Specifically, the method was applied for coniferous tree species due to their well-defined geometric shape. It is worth mentioning that the stem detection method introduced in Chapter 3 can be used to improve the reliability of the local maxima and later the Normalized Cut segmentation results. Chapter 5 explores the potential of multispectral lidar data fused with aerial imagery for tree species classification. In particular, the findings of that chapter demonstrate that the radiometric features extracted from multispectral lidar data have significantly improved the classification accuracy for four tree classes up to $78 \%$. However, from a practical point of view this is not yet satisfactory. New collinear multispectral lidar might help to increase the classification accuracy.

From a practical point of view, the findings of this thesis show a clear improvement of the single tree segmentation for coniferous trees. The adaptive approach introduced in this study can reduce the number of over/under- 
segmented coniferous trees; however, the method needs to be improved for broad-leaf trees. One overall solution to segment broad-leaf trees accurately is to use data acquired under leaf-off conditions. A novel solution of this thesis is to apply the stem detection method (Chapter 3) to high point density ALS data. The outcomes of the thesis suggest that the single tree positions extracted from the stem detection approach could provide complementary information for the detection of local maxima (see Fig.6.3). This can be counted as a new approach for the segmentation of broad-leaf trees. Accurate estimation of the number of trees, particularly broad-leaf trees, is valuable for a variety of forest inventory purposes and applications. In addition, these procedures should be further investigated in order to detect unrecognized trees in the lower and intermediate canopy layers.

The considerations in this thesis show that it is neither possible nor meaningful to completely replace field inventories by remote sensing methods. However, combining the benefits of each method provides a better information density at the same or lower cost for the data collection procedures. The advantage lies in remote sensing data due to the comprehensive coverage ability of important forest structural parameters, which subsequently optimizes the inventory process. Moreover, to capture the necessary ground reference data for calibration of the remote sensing data field inventory is still required. However, one disadvantage of laser scanning systems lies in the high cost of acquisition over large areas. Further approaches at regional and global scales can benefit from lidar data when example projects such as national lidar programs are in operation.

Evaluations of single-tree based approaches for retrieval of forest structural variables which are conducted in this framework can be later used to capture the available degrees of the cost effectiveness of methods for landscape-level forest inventories. Area-based and single tree-based methods are mainly different in terms of required data and computational expenses, especially for area-wide inventories across larger spatial scales. Previous studies on the functionality of both methods mentioned their general practicality for large scale (regional or local) forest inventories in boreal forests (Yu et al., 2011). In case of the non-managed, natural condition of the Bavarian Forest National Park, this point is confirmed by studies such as Latifi et al. (2015a) including the advantages and disadvantages of each method. The methods developed in the context of this thesis for temperate forests are successful in extracting structural attributes at single tree level. However, further investigations are required for area-based practicality at local or global scales. These drawbacks are motivations for future investigations on the additional use of spectral information in a same spatial resolution as the lidar data. However, this can lead to total higher efforts for developing practical and area-wide algorithms for analysis of forest structural attributes similar to those that have been experimentally tested in small sites within the Bavarian Forest National Park in this thesis. Furthermore, assessing forest structure at local or global scale is necessary for addressing the issue of sustainable management of forest resources. To achieve this goal and to have more cost-effective approaches, relying on space-borne lidar missions is acknowledged to be a highly relevant solution. 
Future experiments combining the area-based and single tree-based approaches in the regions consisting of mature and young tree structures can provide a further potential for practical forest inventories based on lidar data. Moreover, concerning the tree species mapping in temperate forests using remote sensing data, the efforts towards practical mapping on national and beyond-national scales could be directed towards using a combination of spectral and structural information offered by multispectral lidar data. Furthermore, the forest management should be aware, that a sufficient number of reference data should be guaranteed beforehand. Besides the practicality, regional-scale applications require more single tree-type classifications which are recently experiencing a rapidly growing trend in the remote sensing literature (Fassnacht et al., 2016), mainly using airborne lidar data which has been meanwhile made available for some national forest inventory programs such as the National Ecological Observatory Network (NEON) in the United States and the Norwegian Mapping Authority. Investing in acquisition of a higher number of reference samples in both managed and natural temperate forests in central Europe does not automatically guarantee a higher quality on estimating forest structural attributes and can only be treated in a more economical way as long as the number of reference plots sufficiently represent the heterogeneity and species diversity existing within the forest types.

\subsection{Further research}

Developing better approaches is required for describing forest structural variables, because they are the basis for monitoring forest structure status and degradation processes. McElhinny et al. (2005) mentioned, that the structural, functional, and compositional attributes of a single tree are highly interdependent because the attributes from any of these groups can be considered as surrogates for others. Therefore, it is important to estimate the forest structure variables accurately. Moreover, a combination of structural attributes added by species diversity and composition are also reported to be useful measures to estimate, for example, the canopy biomass and vertical complexity of a tree (Davey, 1984).

The current implementation of the methods in this thesis is based on the classifier training step. The classification is done through a machine learning approach. The machine learning methods are not an optimal solution if detailed forest structure analysis is concerned. Deep learning approaches for 3D point clouds will provide a new way towards better forestry approaches. Improved research on developing automatic classification solutions such as deep learning using neural networks is currently available for the purpose of indoor mapping, and partly for vegetation analysis. It would be interesting to evaluate how the contribution of deep learning approaches could find a role in forest structure analysis. As a recent development at Stanford University, an open-source framework for semantic segmentation is now available (PointNet++).

Furthermore, the development of new sensors will not only benefit the remote sensing approaches, but will also improve forest structure analysis. 


\section{Bibliography}

Al-Subaihi, I., Watson, G., 2004. The use of the 11 and $1 \infty$ norms in fitting parametric curves and surfaces to data. Applied Numerical Analysis \& Computational Mathematics 1 (2), 363-376.

Amiri, N., Heurich, M., Krzystek, P., Skidmore, A. K., 2018a. Feature relevance assessment of multispectral airborne lidar data for tree species classification. ISPRS - International Archives of the Photogrammetry, Remote Sensing and Spatial Information Sciences XLII-3, 31-34.

Amiri, N., Polewski, P., Heurich, M., Krzystek, P., Skidmore, A. K., 2018b. Adaptive stopping criterion for top-down segmentation of als point clouds in temperate coniferous forests. ISPRS Journal of Photogrammetry and Remote Sensing 141, 265-274.

Amiri, N., Polewski, P., Yao, W., Krzystek, P., Skidmore, A. K., 2017. Detection of single tree stems in forested areas from high density als point clouds using 3d shape descriptors. ISPRS Annals of Photogrammetry, Remote Sensing and Spatial Information Sciences IV-2/W4, 35-42.

Amiri, N., Yao, W., Heurich, M., Krzystek, P., Skidmore, A. K., 2016. Estimation of regeneration coverage in a temperate forest by $3 \mathrm{~d}$ segmentation using airborne laser scanning data. International journal of applied earth observation and geoinformation 52, 252-262.

Asner, G. P., Martin, R. E., 2016. Spectranomics: Emerging science and conservation opportunities at the interface of biodiversity and remote sensing. Global Ecology and Conservation 8, 212-219.

Axelsson, A., Lindberg, E., Olsson, H., 2018. Exploring multispectral als data for tree species classification. Remote Sensing 10 (2), 183-198.

Baldeck, C. A., Asner, G. P., Martin, R. E., Anderson, C. B., Knapp, D. E., Kellner, J. R., Wright, S. J., 07 2015. Operational tree species mapping in a diverse tropical forest with airborne imaging spectroscopy. PLOS ONE $10(7), 1-21$.

Barbati, A., Corona, P., Marchetti, M., 2007. A forest typology for monitoring sustainable forest management: the case of european forest types. Plant Biosystems 141 (1), 93-103.

Bässler, C., Förster, B., Moning, C., Müller, J., 2008. The bioklim-project: biodiversity research between climate change and wilding in a temperate 
montane forest-the conceptual framework. Waldökologie, Landschaftsforschung und Naturschutz 7, 21-33.

Bataineh, A. L., Oswald, B. P., Bataineh, M. M., Williams, H. M., Coble, D. W., 2006. Changes in understory vegetation of a ponderosa pine forest in northern arizona 30 years after a wildfire. Forest Ecology and Management 235 (1-3), 283-294.

Berk, A., Bernstein, L., Anderson, G., Acharya, P., Robertson, D., Chetwynd, J., Adler-Golden, S., 1998. Modtran cloud and multiple scattering upgrades with application to aviris. Remote sensing of Environment 65 (3), 367-375.

Brandtberg, T., 2007. Classifying individual tree species under leaf-off and leaf-on conditions using airborne lidar. ISPRS Journal of Photogrammetry and Remote Sensing 61 (5), 325-340.

Brandtberg, T., Warner, T. A., Landenberger, R. E., McGraw, J. B., 2003. Detection and analysis of individual leaf-off tree crowns in small footprint, high sampling density lidar data from the eastern deciduous forest in north america. Remote Sensing of Environment 85 (3), 290-303.

Braun-Blanquet, J., 1928. Pflanzensoziologie. Springer, Berlin, Springer.

Breiman, L., 2001. Random forests. Machine Learning 45 (1), 5-32.

Briese, C., Höfle, B., Lehner, H., Wagner, W., Pfennigbauer, M., Ullrich, A., 2008. Calibration of full-waveform airborne laser scanning data for object classification. In: Laser Radar Technology and Applications XIII. Vol. 6950. International Society for Optics and Photonics, pp. 6950-6958.

Brolly, G., Király, G., 2009. Algorithms for stem mapping by means of terrestrial laser scanning. Acta Silvatica et Lignaria Hungarica 5, 119-130.

Budei, B. C., St-Onge, B., Hopkinson, C., Audet, F.-A., 2018. Identifying the genus or species of individual trees using a three-wavelength airborne lidar system. Remote Sensing of Environment 204, 632-647.

Cailleret, M., Heurich, M., Bugmann, H., 2014. Reduction in browsing intensity may not compensate climate change effects on tree species composition in the bavarian forest national park. Forest ecology and management 328, 179-192.

Cailliez, F., Alder, D., et al., 1980. Forest volume estimation and yield prediction Vol 1-Volume estimation; Vol. 2-Yield prediction. No. 22.

Cao, L., Coops, N. C., Innes, J. L., Dai, J., Ruan, H., She, G., 2016. Tree species classification in subtropical forests using small-footprint fullwaveform lidar data. International Journal of Applied Earth Observation and Geoinformation 49, 39-51.

Chambers, D., Périé, C., Casajus, N., de Blois, S., 2013. Challenges in modelling the abundance of 105 tree species in eastern north america using climate, edaphic, and topographic variables. Forest Ecology and Management 291, 20-29.

Chang, A., Eo, Y., Kim, Y., Kim, Y., 2013. Identification of individual tree crowns from lidar data using a circle fitting algorithm with local maxima and minima filtering. Remote sensing letters 4 (1), 29-37. 
Comaniciu, D., Meer, P., 2002. Mean shift: A robust approach toward feature space analysis. IEEE Transactions on Pattern Analysis and Machine Intelligence 24 (5).

Congalton, R. G., 1991. A review of assessing the accuracy of classifications of remotely sensed data. Remote sensing of environment 37 (1), 35-46.

Coppin, P. R., Bauer, M. E., 1996. Digital change detection in forest ecosystems with remote sensing imagery. Remote sensing reviews 13 (3-4), 207-234.

Corona, P., Chirici, G., McRoberts, R. E., Winter, S., Barbati, A., 2011. Contribution of large-scale forest inventories to biodiversity assessment and monitoring. Forest Ecology and Management 262 (11), 2061-2069.

Dai, M., Newman, T. S., Cao, C., 2007. Least-squares-based fitting of paraboloids. Pattern Recognition 40 (2), 504-515.

Dalponte, M., Bruzzone, L., Gianelle, D., 2012. Tree species classification in the southern alps based on the fusion of very high geometrical resolution multispectral/hyperspectral images and lidar data. Remote Sensing of Environment 123, 258-270.

Davey, S., 1984. Habitat preferences of arboreal marsupials within a coastal forest in southern new south wales. Possums and gliders, 509-516.

Dersch, S., 2018. 3D-Segmentation of single trees in high density ALS point clouds using prior knowledge of stem positions. Master's thesis, Munich University of Applied Sciences, Germany.

Ellison, A. M., Bank, M. S., Clinton, B. D., Colburn, E. A., Elliott, K., Ford, C. R., Foster, D. R., Kloeppel, B. D., Knoepp, J. D., Lovett, G. M. et al., 2005. Loss of foundation species: consequences for the structure and dynamics of forested ecosystems. Frontiers in Ecology and the Environment $3(9), 479-486$.

Erikson, M., 2004. Species classification of individually segmented tree crowns in high-resolution aerial images using radiometric and morphologic image measures. Remote Sensing of Environment 91 (3-4), 469-477.

Eskelson, B. N. I., Madsen, L., Hagar, J., Temesgen, H., 2011. Estimating riparian understory vegetation cover with beta regression and copula models. Forest Science 57 (3), 212-221.

Falkowski, M. J., Evans, J. S., Martinuzzi, S., Gessler, P. E., Hudak, A. T., 2009. Characterizing forest succession with lidar data: An evaluation for the inland northwest, usa. Remote Sensing of Environment 113 (5), 946-956.

Fassnacht, F. E., Latifi, H., Stereńczak, K., Modzelewska, A., Lefsky, M., Waser, L. T., Straub, C., Ghosh, A., 2016. Review of studies on tree species classification from remotely sensed data. Remote Sensing of Environment $186,64-87$.

Féret, J.-B., Asner, G. P., 2013. Tree species discrimination in tropical forests using airborne imaging spectroscopy. IEEE Transactions on Geoscience and Remote Sensing 51 (1), 73-84. 
Ferraz, A., Bretar, F., Jacquemoud, S., Gonalves, G., Pereira, L., Tom, M., Soares, P., 2012. 3-d mapping of a multi-layered mediterranean forest using ALS data. Remote Sensing of Environment 121, 210-223.

Fischler, M. A., Bolles, R. C., 1981. Random sample consensus: a paradigm for model fitting with applications to image analysis and automated cartography. Communications of the ACM 24 (6), 381-395.

Franklin, J. F., Denison, W., McKee, A., Maser, C., Sedell, J., Swanson, F., Juday, G., et al., 1981. Ecological characteristics of old-growth douglas-fir forests. Gen. Tech. Rep. PNW-GTR-118. Portland, OR: US Department of Agriculture, Forest Service, Pacific Northwest Research Station. 48 p 118.

Franklin, J. F., Spies, T. A., Van Pelt, R., Carey, A. B., Thornburgh, D. A., Berg, D. R., Lindenmayer, D. B., Harmon, M. E., Keeton, W. S., Shaw, D. C., et al., 2002. Disturbances and structural development of natural forest ecosystems with silvicultural implications, using douglas-fir forests as an example. Forest Ecology and Management 155 (1-3), 399-423.

Ghosh, A., Fassnacht, F. E., Joshi, P., Koch, B., 2014. A framework for mapping tree species combining hyperspectral and lidar data: Role of selected classifiers and sensor across three spatial scales. International Journal of Applied Earth Observation and Geoinformation 26, 49-63.

Gong, W., Sun, J., Shi, S., Yang, J., Du, L., Zhu, B., Song, S., 2015. Investigating the potential of using the spatial and spectral information of multispectral lidar for object classification. Sensors 15 (9), 21989-22002.

González-Ferreiro, E., Diéguez-Aranda, U., Barreiro-Fernández, L., Buján, S., Barbosa, M., Suárez, J. C., Bye, I. J., Miranda, D., 2013. A mixed pixel-and region-based approach for using airborne laser scanning data for individual tree crown delineation in pinus radiata d. don plantations. International journal of remote sensing 34 (21), 7671-7690.

Guo, L., Chehata, N., Mallet, C., Boukir, S., 2011. Relevance of airborne lidar and multispectral image data for urban scene classification using random forests. ISPRS Journal of Photogrammetry and Remote Sensing 66 (1), 56-66.

Guyon, I., Elisseeff, A., 2003. An introduction to variable and feature selection. Journal of machine learning research 3, 1157-1182.

Hackel, T., Wegner, J. D., Schindler, K., 2016. Fast semantic segmentation of $3 \mathrm{~d}$ point clouds with strongly varying density. ISPRS Annals of Photogrammetry, Remote Sensing \& Spatial Information Sciences 3 (3).

Haralick, R. M., Shanmugam, K., 1973. Textural features for image classification. IEEE Transactions on systems, man, and cybernetics 3 (6), 610-621.

Hardiman, B. S., Bohrer, G., Gough, C. M., Curtis, P. S., 2013. Canopy structural changes following widespread mortality of canopy dominant trees. Forests 4 (3), 537-552.

URL http://www.mdpi.com/1999-4907/4/3/537 
Harding, D. J., Dabney, P. W., Valett, S., 2011. Polarimetric, two-color, photon-counting laser altimeter measurements of forest canopy structure. In: International Symposium on Lidar and Radar Mapping 2011: Technologies and Applications. Vol. 8286. International Society for Optics and Photonics, p. 828629 .

Hastie, T., Tibshirani, R., Friedman, J., 2001. The Elements of Statistical Learning. Springer.

Heinzel, J., Huber, M. O., 2016. Detecting tree stems from volumetric TLS data in forest environments with rich understory. Remote Sensing 9 (1), 9 .

Heinzel, J., Koch, B., 2011. Exploring full-waveform lidar parameters for tree species classification. International Journal of Applied Earth Observation and Geoinformation 13 (1), 152-160.

Heinzel, J., Koch, B., 2012. Investigating multiple data sources for tree species classification in temperate forest and use for single tree delineation. International Journal of Applied Earth Observation and Geoinformation $18,101-110$.

Hen, Y. W., Khalid, M., Yusof, R., 2007. Face verification with gabor representation and support vector machines. In: Modelling \& Simulation, 2007. AMS'07. First Asia International Conference on. IEEE, pp. 451-459.

Henning, J. G., Radtke, P. J., 2006. Detailed stem measurements of standing trees from ground-based scanning lidar. Forest Science 52 (1), 67-80.

Heurich, M., 2006. Evaluierung und Entwicklung von Methoden zur automatisierten Erfassung von Waldstrukturen aus Daten flugzeuggetragener Fernerkundungssensoren. Ph.D. thesis, Technische Universität München.

Heurich, M., 2008. Automatic recognition and measurement of single trees based on data from airborne laser scanning over the richly structured natural forests of the bavarian forest national park. Forest Ecology and Management 255 (7), 2416-2433.

Heurich, M., Beudert, B., Rall, H., Křenová, Z., 2010. National Parks as Model Regions for Interdisciplinary Long-Term Ecological Research: The Bavarian Forest and Šumavá National Parks Underway to Transboundary Ecosystem Research. Springer Netherlands, book section 23, pp. 327-344.

Hill, R. A., Broughton, R. K., 2009. Mapping the understorey of deciduous woodland from leaf-on and leaf-off airborne lidar data: A case study in lowland britain. ISPRS Journal of Photogrammetry and Remote Sensing 64 (2), 223-233.

Hill, S., Latifi, H., Heurich, M., Müller, J., 2017. Individual-tree-and standbased development following natural disturbance in a heterogeneously structured forest: A lidar-based approach. Ecological Informatics 38, 1225.

Hilmers, T., Friess, N., Bässler, C., Heurich, M., Brandl, R., Pretzsch, H., Seidl, R., Müller, J., 2018. Biodiversity along temperate forest succession. Journal of Applied Ecology 55 (6), 2756-2766. 
Hirschmüller, H., 2008. Stereo processing by semiglobal matching and mutual information. IEEE Transactions on pattern analysis and machine intelligence 30 (2), 328-341.

Höfle, B., Hollaus, M., Hagenauer, J., 2012. Urban vegetation detection using radiometrically calibrated small-footprint full-waveform airborne lidar data. ISPRS Journal of Photogrammetry and Remote Sensing 67, 134 - 147.

Höfle, B., Hollaus, M., Lehner, H., Pfeifer, N., Wagner, W., 2008. Area-based parameterization of forest structure using full-waveform airborne laser scanning data. silvilaser 2008. In: 8th International Conference on LiDAR Applications in forest assessment and inventory, Heriot-Watt University, Edinburgh, UK. pp. 17-19.

Höfle, B., Pfeifer, N., 2007. Correction of laser scanning intensity data: Data and model-driven approaches. ISPRS Journal of Photogrammetry and Remote Sensing 62 (6), 415 - 433.

Holmgren, J., Persson, A., 2004. Identifying species of individual trees using airborne laser scanner. Remote Sensing of Environment 90 (4), 415-423.

Holmgren, J., Persson, Å., Söderman, U., 2008. Species identification of individual trees by combining high resolution lidar data with multi-spectral images. International Journal of Remote Sensing 29 (5), 1537-1552.

Hopkinson, C., 2007. The influence of flying altitude, beam divergence, and pulse repetition frequency on laser pulse return intensity and canopy frequency distribution. Canadian Journal of Remote Sensing 33 (4), 312324 .

Hopkinson, C., Chasmer, L., Gynan, C., Mahoney, C., Sitar, M., 2016. Multisensor and multispectral lidar characterization and classification of a forest environment. Canadian Journal of Remote Sensing 42 (5), 501-520.

Hovi, A., Korhonen, L., Vauhkonen, J., Korpela, I., 2016. Lidar waveform features for tree species classification and their sensitivity to tree-and acquisition related parameters. Remote Sensing of Environment 173, 224237.

Hu, B., Li, J., Jing, L., Judah, A., 2014. Improving the efficiency and accuracy of individual tree crown delineation from high-density lidar data. International Journal of Applied Earth Observation and Geoinformation $26,145-155$.

Husch, B., Beers, T., Kershaw, J., 2002. Forest Mensuration. Ecology (John Wiley and Sons). John Wiley \& Sons.

Hyyppä, J., Hyyppä, H., Inkinen, M., Engdahl, M., Linko, S., Zhu, Y. H., 2000. Accuracy comparison of various remote sensing data sources in the retrieval of forest stand attributes. Forest Ecology and Management 128 (1-2), 109-120.

Hyyppä, J., Hyyppä, H., Leckie, D., Gougeon, F., Yu, X., Maltamo, M., 2008. Review of methods of small-footprint airborne laser scanning for extracting forest inventory data in boreal forests. International Journal of Remote Sensing 29 (5), 1339-1366. 
Hyyppä, J., Kelle, O., Lehikoinen, M., Inkinen, M., 2001. A segmentationbased method to retrieve stem volume estimates from 3-d tree height models produced by laser scanners. IEEE Transactions on geoscience and remote sensing 39 (5), 969-975.

Hyyppä, J., Yu, X., Hyyppä, H., Vastaranta, M., Holopainen, M., Kukko, A., Kaartinen, H., Jaakkola, A., Vaaja, M., Koskinen, J., et al., 2012. Advances in forest inventory using airborne laser scanning. Remote Sensing 4 (5), 1190-1207.

Immitzer, M., Atzberger, C., Koukal, T., 2012. Tree species classification with random forest using very high spatial resolution 8-band worldview-2 satellite data. Remote Sensing 4 (9), 2661-2693.

Jakubowski, M. K., Li, W., Guo, Q., Kelly, M., 2013. Delineating individual trees from lidar data: A comparison of vector-and raster-based segmentation approaches. Remote Sensing 5 (9), 4163-4186.

Janssen, L. L., Huurneman, G., 2000. Principles of remote sensing. ITC, International Institute for Aerospace Survey and Earth Sciences.

Jaskierniak, D., Lane, P. N. J., Robinson, A., Lucieer, A., 2011. Extracting lidar indices to characterise multilayered forest structure using mixture distribution functions. Remote Sensing of Environment 115 (2), 573-585.

Jones, T. G., Coops, N. C., Sharma, T., 2010. Assessing the utility of airborne hyperspectral and lidar data for species distribution mapping in the coastal pacific northwest, canada. Remote Sensing of Environment 114 (12), 2841-2852.

Kashani, A. G., Olsen, M. J., Parrish, C. E., Wilson, N., 2015. A review of lidar radiometric processing: From ad hoc intensity correction to rigorous radiometric calibration. Sensors 15 (11), 28099-28128.

Kasparian, J., Rodriguez, M., Méjean, G., Yu, J., Salmon, E., Wille, H., Bourayou, R., Frey, S., André, Y.-B., Mysyrowicz, A., Sauerbrey, R., Wolf, J.-P., Wöste, L., 2003. White-light filaments for atmospheric analysis. Science 301 (5629), 61-64.

Kerns, B. K., Ohmann, J. L., 2004. Evaluation and prediction of shrub cover in coastal oregon forests (usa). Ecological Indicators 4 (2), 83-98.

Khosravipour, A., Skidmore, A. K., Isenburg, M., Wang, T., Hussin, Y. A., 2014. Generating pit-free canopy height models from airborne lidar. Photogrammetric Engineering \& Remote Sensing 80 (9), 863-872.

Kiener, H., Hulein, M., Englmaier, K. H., 2008. Management im nationalpark bayerischer wald. Natura 2000.

Killinger, D. K., Meyuk, N., 1987. Laser remote sensing of the atmosphere. Science 235 (4784), 37-45.

Kim, S., 2008. Individual tree species identification using lidar-derived crown structures and intensity data. Ph.D. thesis, University of Washington.

Kim, S., Hinckley, T., Briggs, D., 2011. Classifying individual tree genera using stepwise cluster analysis based on height and intensity metrics derived from airborne laser scanner data. Remote Sensing of Environment 115 (12), 3329-3342. 
Kim, S., McGaughey, R. J., Andersen, H.-E., Schreuder, G., 2009. Tree species differentiation using intensity data derived from leaf-on and leafoff airborne laser scanner data. Remote Sensing of Environment 113 (8), $1575-1586$.

Koch, B., Straub, C., Dees, M., Wang, Y., Weinacker, H., 2009. Airborne laser data for stand delineation and information extraction. International Journal of Remote Sensing 30 (4), 935-963.

Köhl, M., Magnussen, S. S., Marchetti, M., 2006. Sampling methods, remote sensing and GIS multiresource forest inventory. Springer Science \& Business Media.

Koop, H., 1989. Forest Dynamics: SILVI-STAR, a Comprehensive Monitoring System. Springer-Verlag.

Korpela, I., Hovi, A., Morsdorf, F., 2012. Understory trees in airborne lidar data selective mapping due to transmission losses and echo-triggering mechanisms. Remote Sensing of Environment 119, 92-104.

Korpela, I., Koskinen, M., Vasander, H., Holopainen, M., Minkkinen, K. 2009. Airborne small-footprint discrete-return lidar data in the assessment of boreal mire surface patterns, vegetation, and habitats. Forest Ecology and Management 258 (7), 1549-1566.

Korpela, I., Ørka, H. O., Hyyppä, J., Heikkinen, V., Tokola, T., 2010a. Range and agc normalization in airborne discrete-return lidar intensity data for forest canopies. ISPRS Journal of Photogrammetry and Remote Sensing 65 (4), 369-379.

Korpela, I., Ørka, H. O., Maltamo, M., Tokola, T., Hyyppä, J., et al., 2010b. Tree species classification using airborne lidar-effects of stand and tree parameters, downsizing of training set, intensity normalization, and sensor type. Silva Fennica 44 (2), 319-339.

Korpela, I., et al., 2004. Individual tree measurements by means of digital aerial photogrammetry.

Krzystek, P., Polewski, P., 2017. Objektbasierte segmentierung und klassifikation von lidar-punktwolken. In: Photogrammetrie und Fernerkundung. Springer, pp. 645-684.

Larson, B. C., Oliver, C. D., 1996. Forest stand dynamics. John Wiley \& Sons.

Latifi, H., 2011. The use of nonparametric methods for small-scale forest inventory by means of multiple remote sensing data sources. Ph.D. thesis, Universität.

Latifi, H., 2012. Characterizing forest structure by means of remote sensing: a review. In: Remote Sensing-Advanced Techniques and Platforms. InTech.

Latifi, H., Fassnacht, F. E., Müller, J., Tharani, A., Dech, S., Heurich, M., 2015a. Forest inventories by lidar data: A comparison of single tree segmentation and metric-based methods for inventories of a heterogeneous temperate forest. International Journal of Applied Earth Observation and Geoinformation 42, 162-174. 
Latifi, H., Heurich, M., Hartig, F., Müller, J., Krzystek, P., Jehl, H., Dech, S., 2015b. Estimating over- and understorey canopy density of temperate mixed stands by airborne lidar data. Forestry, 1-13.

Latifi, H., Hill, S., Schumann, B., Heurich, M., Dech, S., 2017. Multi-model estimation of understorey shrub, herb and moss cover in temperate forest stands by laser scanner data. Forestry: An International Journal of Forest Research 90 (4), 496-514.

Leckie, D. G., Gougeon, F. A., Tinis, S., Nelson, T., Burnett, C. N., Paradine, D., 2005. Automated tree recognition in old growth conifer stands with high resolution digital imagery. Remote Sensing of Environment 94 (3), 311-326.

Leckie, D. G., Gougeon, F. A., Walsworth, N., Paradine, D., 2003. Stand delineation and composition estimation using semi-automated individual tree crown analysis. Remote Sensing of Environment 85 (3), 355-369.

Lee, H., Slatton, K. C., Roth, B., Cropper Jr, W., 2010. Adaptive clustering of airborne lidar data to segment individual tree crowns in managed pine forests. International Journal of Remote Sensing 31 (1), 117-139.

Lefsky, M. A., Cohen, W., Acker, S., Parker, G. G., Spies, T., Harding, D., 1999. Lidar remote sensing of the canopy structure and biophysical properties of douglas-fir western hemlock forests. Remote sensing of environment 70 (3), 339-361.

Lefsky, M. A., Cohen, W. B., Harding, D. J., Parker, G. G., Acker, S. A., Gower, S. T., 2002a. Lidar remote sensing of above-ground biomass in three biomes. Global ecology and biogeography 11 (5), 393-399.

Lefsky, M. A., Cohen, W. B., Parker, G. G., Harding, D. J., 2002b. Lidar remote sensing for ecosystem studies. BioScience 52 (1), 19-30.

Li, J., Hu, B., Noland, T. L., 2013. Classification of tree species based on structural features derived from high density lidar data. Agricultural and forest meteorology 171, 104-114.

Li, W., Guo, Q., Jakubowski, M. K., Kelly, M., 2012. A new method for segmenting individual trees from the lidar point cloud. Photogrammetric Engineering \& Remote Sensing 78 (1), 75-84.

Liang, X., Hyyppä, J., 2013. Automatic stem mapping by merging several terrestrial laser scans at the feature and decision levels. Sensors 13 (2), 1614-1634.

Liang, X., Litkey, P., Hyyppä, J., Kaartinen, H., Vastaranta, M., Holopainen, M., 2012. Automatic stem mapping using single-scan terrestrial laser scanning. IEEE Transactions on Geoscience and Remote Sensing 50 (2), 661-670.

Lim, K., Treitz, P., Wulder, M., St-Onge, B., Flood, M., 2003. Lidar remote sensing of forest structure. Progress in physical geography 27 (1), 88-106.

Lin, Y., Herold, M., 2016. Tree species classification based on explicit tree structure feature parameters derived from static terrestrial laser scanning data. Agricultural and Forest meteorology 216, 105-114. 
Lin, Y., Hyyppä, J., Jaakkola, A., May 2011. Mini-uav-borne lidar for fine-scale mapping. IEEE Geoscience and Remote Sensing Letters 8 (3), 426-430.

Lindberg, E., Briese, C., Doneus, M., Hollaus, M., Schroiff, A., Pfeifer, N., 2015. Multi-wavelength airborne laser scanning for characterization of tree species. Proceedings of SilviLaser, 271-273.

Lindberg, E., Holmgren, J., Olofsson, K., Olsson, H., 2012. Estimation of stem attributes using a combination of terrestrial and airborne laser scanning. European Journal of Forest Research 131 (6), 1917-1931.

Liu, H., Motoda, H., Setiono, R., Zhao, Z., 2010. Feature selection: An ever evolving frontier in data mining. In: Feature Selection in Data Mining. pp. $4-13$.

Liu, Y., Wang, W., 2008. A revisit to least squares orthogonal distance fitting of parametric curves and surfaces. In: International Conference on Geometric Modeling and Processing. Springer, pp. 384-397.

Lovell, J., Jupp, D. L., Culvenor, D., Coops, N., 2003. Using airborne and ground-based ranging lidar to measure canopy structure in australian forests. Canadian Journal of Remote Sensing 29 (5), 607-622.

Mallet, C., Bretar, F., 2009. Full-waveform topographic lidar: State-of-the-art. ISPRS Journal of photogrammetry and remote sensing 64 (1), 1-16.

Mallet, C., Bretar, F., Soergel, U., 2008. Analysis of full-waveform lidar data for classification of urban areas. Photogrammetrie Fernerkundung Geoinformation 5, 337-349.

Maltamo, M., Bollandsås, O., Vauhkonen, J., Breidenbach, J., Gobakken, T., Næsset, E., 2010. Comparing different methods for prediction of mean crown height in norway spruce stands using airborne laser scanner data. Forestry 83 (3), 257-268.

Maltamo, M., Mehtätalo, L., Vauhkonen, J., Packalén, P., 2012. Predicting and calibrating tree attributes by means of airborne laser scanning and field measurements. Canadian Journal of Forest Research 42 (11), 1896-1907.

Maltamo, M., Næsset, E., Vauhkonen, J., 2014. Forestry applications of airborne laser scanning. Concepts and case studies. Managing Forest Ecosystems book series 27,460 .

Maltamo, M., Packalén, P., Yu, X., Eerikäinen, K., Hyyppä, J., Pitkänen, J., 2005. Identifying and quantifying structural characteristics of heterogeneous boreal forests using laser scanner data. Forest Ecology and Management $216(1-3), 41-50$.

Mas, J.-F., 1999. Monitoring land-cover changes: a comparison of change detection techniques. International journal of remote sensing 20 (1), 139 152.

McElhinny, C., Gibbons, P., Brack, C., Bauhus, J., 2005. Forest and woodland stand structural complexity: its definition and measurement. Forest Ecology and Management 218 (1-3), 1-24. 
McKenzie, D., Halpern, C. B., Nelson, C. R., 2000. Overstory influences on herb and shrub communities in mature forests of western washington, usa. Canadian Journal of Forest Research 30 (10), 1655-1666.

McMillin, J. D., Allen, K. K., 2003. Effects of douglas-fir beetle (coleoptera: Scolytidae) infestations on forest overstory and understory conditions in western wyoming. Western North American Naturalist, 498-506.

Moffiet, T., Mengersen, K., Witte, C., King, R., Denham, R., 2005. Airborne laser scanning: Exploratory data analysis indicates potential variables for classification of individual trees or forest stands according to species. ISPRS Journal of Photogrammetry and Remote Sensing 59 (5), 289-309.

Morsdorf, F., Kötz, B., Meier, E., Itten, K., Allgöwer, B., 2006. Estimation of lai and fractional cover from small footprint airborne laser scanning data based on gap fraction. Remote Sensing of Environment 104 (1), 50-61.

Morsdorf, F., Mårell, A., Koetz, B., Cassagne, N., Pimont, F., Rigolot, E. Allgöwer, B., 2010. Discrimination of vegetation strata in a multi-layered mediterranean forest ecosystem using height and intensity information derived from airborne laser scanning. Remote Sensing of Environment 114 (7), 1403-1415

Morsdorf, F., Meier, E., Kötz, B., Itten, K. I., Dobbertin, M., Allgöwer, B. 2004. Lidar-based geometric reconstruction of boreal type forest stands at single tree level for forest and wildland fire management. Remote Sensing of Environment 92 (3), 353-362.

Morsy, S., Shaker, A., El-Rabbany, A., LaRocque, P. E., 2016. Airborne multispectral lidar data for land-cover classification and land/water mapping using different spectral indexes. ISPRS Annals of Photogrammetry, Remote Sensing \& Spatial Information Sciences 3 (3).

Mund, J.-P., Wilke, R., Körner, M., Schultz, A., 2015. Detecting multi-layered forest stands using high density airborne lidar data. J. Geogr. Inf. Sci 1, $178-188$

Nakamura, G., et al., 1996. Harvesting forest biomass reduces wildfire fuel. California Agriculture 50 (2), 13-16.

Nevalainen, O., Honkavaara, E., Tuominen, S., Viljanen, N., Hakala, T., Yu, X., Hyyppä, J., Saari, H., Pölönen, I., Imai, N. N., et al., 2017. Individual tree detection and classification with uav-based photogrammetric point clouds and hyperspectral imaging. Remote Sensing 9 (3), 185-219.

Nie, F., Ding, C., Luo, D., Huang, H., 2010. Improved minmax cut graph clustering with nonnegative relaxation. In: Joint European Conference on Machine Learning and Knowledge Discovery in Databases. Springer, pp. $451-466$.

Olofsson, K., Holmgren, J., Olsson, H., 2014. Tree stem and height measurements using terrestrial laser scanning and the ransac algorithm. Remote sensing 6 (5), 4323-4344.

Optech, 2015. Optech titan. http://www . teledyneoptech.com/index.php/ product/titan/, accessed: 2018-04-28. 
Ørka, H. O., Næsset, E., Bollandsås, O. M., 2009. Classifying species of individual trees by intensity and structure features derived from airborne laser scanner data. Remote Sensing of Environment 113 (6), 1163-1174.

Ørka, H. O., Næsset, E., Bollandsås, O. M., 2010. Effects of different sensors and leaf-on and leaf-off canopy conditions on echo distributions and individual tree properties derived from airborne laser scanning. Remote Sensing of Environment 114 (7), 1445-1461.

Packalén, P., Suvanto, A., Maltamo, M., 2009. A two stage method to estimate species-specific growing stock. Photogrammetric engineering \& remote sensing 75 (12), 1451-1460.

Paletto, A., Tosi, V., 2009. Forest canopy cover and canopy closure: comparison of assessment techniques. European Journal of Forest Research 128 (3), 265-272.

Persson, A., Holmgren, J., Söderman, U., 2002. Detecting and measuring individual trees using an airborne laser scanner. Photogrammetric Engineering and Remote Sensing 68 (9), 925-932.

Pfeifer, N., Winterhalder, D., 2004. Modelling of tree cross sections from terrestrial laser scanning data with free-form curves. International Archives of Photogrammetry, remote sensing and spatial information sciences 36 (Part 8), W2.

Pirotti, F., 2011. Analysis of full-waveform lidar data for forestry applications: a review of investigations and methods. iForest-Biogeosciences and Forestry $4(3), 100$.

Polewski, P., Erickson, A., Yao, W., Coops, N., Krzystek, P., Stilla, U., 2016. Object-based coregistration of terrestrial photogrammetric and als point clouds in forested areas. ISPRS Annals of Photogrammetry, Remote Sensing and Spatial Information Sciences, 347-354.

Polewski, P., Yao, W., Heurich, M., Krzystek, P., Stilla, U., 2015a. Detection of fallen trees in ALS point clouds using a normalized cut approach trained by simulation. ISPRS Journal of Photogrammetry and Remote Sensing $105,252-271$.

Polewski, P., Yao, W., Heurich, M., Krzystek, P., Stilla, U., 2015b. Detection of single standing dead trees from aerial color infrared imagery by segmentation with shape and intensity priors. In: Pia15 + Hrigi15-Joint Isprs Conference, Vol. Ii. No. W4. International Society for Photogrammetry and Remote Sensing, pp. 181-188.

Polewski, P., Yao, W., Heurich, M., Krzystek, P., Stilla, U., 2017. A votingbased statistical cylinder detection framework applied to fallen tree mapping in terrestrial laser scanning point clouds. ISPRS Journal of Photogrammetry and Remote Sensing 129, 118-130.

Polewski, P. P., 2017. Reconstruction of standing and fallen single dead trees in forested areas from lidar data and aerial imagery. Ph.D. thesis, Technische Universität München. 
Poorter, L., 1999. Growth responses of 15 rain-forest tree species to a light gradient: the relative importance of morphological and physiological traits. Functional ecology 13 (3), 396-410.

Puttonen, E., Suomalainen, J., Hakala, T., Räikkönen, E., Kaartinen, H. Kaasalainen, S., Litkey, P., 2010. Tree species classification from fused active hyperspectral reflectance and lidar measurements. Forest Ecology and Management 260 (10), 1843-1852.

Pyysalo, U., Hyyppä, H., 2002. Reconstructing tree crowns from laser scanner data for feature extraction. International Archives Of Photogrammetry Remote Sensing and Spatial Information Sciences 34 (3/B), 218-221.

Razak, K. A., Straatsma, M., Van Westen, C., Malet, J.-P., De Jong, S., 2011. Airborne laser scanning of forested landslides characterization: Terrain model quality and visualization. Geomorphology 126 (1), 186-200.

Reitberger, J., 2010. 3D-Segmentierung von Einzelbäumen und Baumartenklassifikation aus Daten flugzeuggetragener Full Waveform Laserscanner. Verlag der Bayerischen Akademie der Wissenschaften.

Reitberger, J., Krzystek, P., Stilla, U., 2007. Combined tree segmentation and stem detection using full waveform lidar data. International Archives of Photogrammetry, Remote Sensing and Spatial Information Sciences 36, $332-337$

Reitberger, J., Krzystek, P., Stilla, U., 2008. Analysis of full waveform lidar data for the classification of deciduous and coniferous trees. International Journal of Remote Sensing 29 (5), 1407-1431.

Reitberger, J., Schnörr, C., Krzystek, P., Stilla, U., 2009. 3d segmentation of single trees exploiting full waveform lidar data. ISPRS Journal of Photogrammetry and Remote Sensing 64 (6), 561-574.

Riegl, 05 2012. LAS extrabytes implementation in RIEGL software. Tech. rep.

Riegl, Nov.2017. Private communication.

Risojević, V., Momić, S., Babić, Z., 2011. Gabor descriptors for aerial image classification. In: International Conference on Adaptive and Natural Computing Algorithms. Springer, pp. 51-60.

Rohde, W.G., O. J. C., 1972. Multispectral sensing of forest tree species. Remote Sensing of Environment 38, 1209-1215.

Roscher, R., Förstner, W., Waske, B., 2012. I 2 vm: incremental import vector machines. Image and Vision Computing 30 (4), 263-278.

Rusu, R. B., Marton, Z. C., Blodow, N., Beetz, M., 2008. Learning informative point classes for the acquisition of object model maps. In: Control, Automation, Robotics and Vision, 2008. ICARCV 2008. 10th International Conference on. IEEE, pp. 643-650.

Savaresi, S. M., Boley, D. L., 2001. On the performance of bisecting k-means and pddp. In: Proceedings of the 2001 SIAM International Conference on Data Mining. SIAM, pp. 1-14. 
Scaioni, M., Hö̈le, B., Baungarten Kersting, A., Barazzetti, L., Previtali, M., Wujanz, D., et al., 2018. Methods from information extraction from lidar intensity data and multispectral lidar technology. In: 2018 ISPRS TC III Mid-Term Symposium on Developments, Technologies and Applications in Remote Sensing. Vol. 42. International Society for Photogrammetry and Remote Sensing, pp. 1503-1510.

Schlerf, M., Atzberger, C., Hill, J., 2005. Remote sensing of forest biophysical variables using hymap imaging spectrometer data. Remote Sensing of Environment 95 (2), 177-194.

Schuster, C., Förster, M., Kleinschmit, B., 2012. Testing the red edge channel for improving land-use classifications based on high-resolution multispectral satellite data. International Journal of Remote Sensing 33 (17), $5583-5599$.

Shang, X., Chazette, P., 2014. Interest of a full-waveform flown uv lidar to derive forest vertical structures and aboveground carbon. Forests 5 (6), $1454-1480$.

Shi, J., Malik, J., 2000. Normalized cuts and image segmentation. IEEE Transactions on Pattern Analysis and Machine Intelligence 22 (8), 888-905.

Shi, Y., Skidmore, A. K., Wang, T., Holzwarth, S., Heiden, U., Pinnel, N., Zhu, X., Heurich, M., 2018b. Tree species classification using plant functional traits from lidar and hyperspectral data. International Journal of Applied Earth Observation and Geoinformation 73, 207 - 219.

Shi, Y., Wang, T., Skidmore, A. K., Heurich, M., 2018a. Important lidar metrics for discriminating forest tree species in central europe. ISPRS Journal of Photogrammetry and Remote Sensing 137, 163 - 174.

Sikora, R., Piramuthu, S., 2007. Framework for efficient feature selection in genetic algorithm based data mining. European Journal of Operational Research 180 (2), 723-737.

Skidmore, A., Pettorelli, 2015. Environmental science: agree on biodiversity metrics to track from space. Nature 523, 403-405.

Solberg, S., Naesset, E., Bollandsas, O. M., 2006. Single tree segmentation using airborne laser scanner data in a structurally heterogeneous spruce forest. Photogrammetric Engineering \& Remote Sensing 72 (12), 13691378.

Somers, B., Asner, G. P., 2014. Tree species mapping in tropical forests using multi-temporal imaging spectroscopy: Wavelength adaptive spectral mixture analysis. International Journal of Applied Earth Observation and Geoinformation 31, 57-66.

St-Onge, B., Budei, B., 2015. Individual tree species identification using the multispectral return intensities of the optech titan lidar system. Proceedings of SilviLaser, 71-73.

Stoker, J. M., Abdullah, Q. A., Nayegandhi, A., Winehouse, J., 2016. Evaluation of single photon and geiger mode lidar for the $3 \mathrm{~d}$ elevation program. Remote Sensing 8 (9), 767. 
Stone, J. N., Porter, J. L., 1998. What is forest structure and how to measure it? Northwest Science 72, 25-26.

Strîmbu, V. F., Strîmbu, B. M., 2015. A graph-based segmentation algorithm for tree crown extraction using airborne lidar data. ISPRS Journal of Photogrammetry and Remote Sensing 104, 30-43.

$\mathrm{Su}$, J. G., Bork, E. W., 2007. Characterization of diverse plant communities in aspen parkland rangeland using lidar data. Applied Vegetation Science 10 (3), 407-416.

Swanson, M. E., Franklin, J. F., Beschta, R. L., Crisafulli, C. M., DellaSala, D. A., Hutto, R. L., Lindenmayer, D. B., Swanson, F. J., 2010. The forgotten stage of forest succession: early-successional ecosystems on forest sites. Frontiers in Ecology and the Environment 9 (2), 117-125.

Swatantran, A., Tang, H., Barrett, T., DeCola, P., Dubayah, R., 2016. Rapid, high-resolution forest structure and terrain mapping over large areas using single photon lidar. Scientific reports 6, 28277.

Toldo, R., Castellani, U., Fusiello, A., Oct 2010. The bag of words approach for retrieval and categorization of $3 \mathrm{~d}$ objects. The Visual Computer 26 (10), $1257-1268$.

Treitz, P., Howarth, P., 2000. High spatial resolution remote sensing data for forest ecosystem classification: an examination of spatial scale. Remote Sensing of Environment 72 (3), 268-289.

Trier, Ø. D., Salberg, A.-B., Kermit, M., Rudjord, Ø., Gobakken, T., Næsset, E., Aarsten, D., 2018. Tree species classification in norway from airborne hyperspectral and airborne laser scanning data. European Journal of Remote Sensing 51 (1), 336-351.

Tuanmu, M.-N., Via, A., Bearer, S., Xu, W., Ouyang, Z., Zhang, H., Liu, J., 2010. Mapping understory vegetation using phenological characteristics derived from remotely sensed data. Remote Sensing of Environment 114 (8), $1833-1844$.

Turner, W., Spector, S., Gardiner, N., Fladeland, M., Sterling, E., Steininger, M., 2003. Remote sensing for biodiversity science and conservation. Trends in ecology \& evolution 18 (6), 306-314.

Ullah, S., Dees, M., Datta, P., Adler, P., Koch, B., 2017. Comparing airborne laser scanning, and image-based point clouds by semi-global matching and enhanced automatic terrain extraction to estimate forest timber volume. Forests 8 (6), 215-230.

Van Der Heijden, F., Duin, R., De Ridder, D., Tax, D. M., 2005. Classification, parameter estimation and state estimation: an engineering approach using MATLAB. John Wiley \& Sons.

Van Laar, A., Akça, A., 2007. Forest mensuration. Vol. 13. Springer Science \& Business Media.

Vastaranta, M., Kankare, V., Holopainen, M., Yu, X., Hyyppä, J., Hyyppä, H., 2012. Combination of individual tree detection and area-based approach in imputation of forest variables using airborne laser data. ISPRS Journal of Photogrammetry and Remote Sensing 67, 73-79. 
Vauhkonen, J., 2010. Estimating crown base height for scots pine by means of the $3 \mathrm{~d}$ geometry of airborne laser scanning data. International Journal of Remote Sensing 31 (5), 1213-1226.

Vauhkonen, J., Ene, L., Gupta, S., Heinzel, J., Holmgren, J., Pitkänen, J., Solberg, S., Wang, Y., Weinacker, H., Hauglin, K. M., et al., 2011. Comparative testing of single-tree detection algorithms under different types of forest. Forestry 85 (1), 27-40.

Vauhkonen, J., Ørka, H. O., Holmgren, J., Dalponte, M., Heinzel, J., Koch, B., 2014. Tree species recognition based on airborne laser scanning and complementary data sources. In: Forestry applications of airborne laser scanning. Springer, pp. 135-156.

Véga, C., Hamrouni, A., El Mokhtari, S., Morel, J., Bock, J., Renaud, J.-P., Bouvier, M., Durrieu, S., 2014. Ptrees: A point-based approach to forest tree extraction from lidar data. International Journal of Applied Earth Observation and Geoinformation 33, 98-108.

Vierling, L. A., Xu, Y., Eitel, J. U., Oldow, J. S., 2013. Shrub characterization using terrestrial laser scanning and implications for airborne lidar assessment. Canadian Journal of Remote Sensing 38 (6), 709-722.

Vosselman, G., Maas, H.-G., 2010. Airborne and terrestrial laser scanning. CRC.

Wagner, W., Hollaus, M., Briese, C., Ducic, V., 2008. 3d vegetation mapping using small-footprint full-waveform airborne laser scanners. International Journal of Remote Sensing 29 (5), 1433-1452.

Wagner, W., Ullrich, A., Ducic, V., Melzer, T., Studnicka, N., 2006. Gaussian decomposition and calibration of a novel small-footprint full-waveform digitising airborne laser scanner. ISPRS journal of Photogrammetry and Remote Sensing 60 (2), 100-112.

Wallace, A., Nichol, C., Woodhouse, I., 2012. Recovery of forest canopy parameters by inversion of multispectral lidar data. Remote Sensing 4 (2), $509-531$.

Wang, D., Hollaus, M., Puttonen, E., Pfeifer, N., 2016. Automatic and selfadaptive stem reconstruction in landslide-affected forests. Remote Sensing 8 (12), 974.

Wang, Y., Weinacker, H., Koch, B., 2008. A lidar point cloud based procedure for vertical canopy structure analysis and 3d single tree modelling in forest. Sensors 8 (6), 3938-3951.

Wang, Z., Boesch, R., Ginzler, C., 2012. Forest delineation of aerial images with gabor wavelets. International journal of remote sensing 33 (7), 21962213.

Waser, L. T., Ginzler, C., Kuechler, M., Baltsavias, E., Hurni, L., 2011. Semi-automatic classification of tree species in different forest ecosystems by spectral and geometric variables derived from airborne digital sensor (ads40) and rc30 data. Remote Sensing of Environment 115 (1), 76-85. 
Waser, L. T., Klonus, S., Ehlers, M., Küchler, M., Jung, A., 2010. Potential of digital sensors for land cover and tree species classifications-a case study in the framework of the dgpf-project. Photogrammetrie-FernerkundungGeoinformation 2010 (2), 141-156.

Watson, G., 2002. On the gauss-newton method for 11 orthogonal distance regression. IMA Journal of Numerical Analysis 22 (3).

Weinmann, M., Jutzi, B., Hinz, S., Mallet, C., 2015a. Semantic point cloud interpretation based on optimal neighborhoods, relevant features and efficient classifiers. ISPRS Journal of Photogrammetry and Remote Sensing $105,286-304$.

Weinmann, M., Jutzi, B., Mallet, C., 2013. Feature relevance assessment for the semantic interpretation of 3d point cloud data. ISPRS Annals of Photogrammetry, Remote Sensing and Spatial Information Sciences II-5/W2.

Weinmann, M., Urban, S., Hinz, S., Jutzi, B., Mallet, C., 2015b. Distinctive $2 \mathrm{~d}$ and $3 \mathrm{~d}$ features for automated large-scale scene analysis in urban areas. Computers \& Graphics 49, 47-57.

White, J. C., Coops, N. C., Wulder, M. A., Vastaranta, M., Hilker, T., Tompalski, P., 2016. Remote sensing technologies for enhancing forest inventories: A review. Canadian Journal of Remote Sensing 42 (5), 619641.

White, J. C., Wulder, M. A., Varhola, A., Vastaranta, M., Coops, N. C., Cook, B. D., Pitt, D., Woods, M., 2013. A best practices guide for generating forest inventory attributes from airborne laser scanning data using an area-based approach. The Forestry Chronicle 89 (6), 722-723.

Wing, B. M., Ritchie, M. W., Boston, K., Cohen, W. B., Gitelman, A., Olsen, M. J., 2012. Prediction of understory vegetation cover with airborne lidar in an interior ponderosa pine forest. Remote Sensing of Environment 124, $730-741$.

Woodhouse, I. H., Nichol, C., Sinclair, P., Jack, J., Morsdorf, F., Malthus, T. J., Patenaude, G., 2011. A multispectral canopy lidar demonstrator project. IEEE Geoscience and Remote Sensing Letters 8 (5), 839-843.

Wright, S., Nocedal, J., 1999. Numerical optimization. Springer Science 35, $67-68$.

Wu, B., Yu, B., Wu, Q., Huang, Y., Chen, Z., Wu, J., 2016. Individual tree crown delineation using localized contour tree method and airborne lidar data in coniferous forests. International Journal of Applied Earth Observation and Geoinformation 52, 82-94.

Wu, K.-L., Yang, M.-S., 2007. Mean shift-based clustering. Pattern Recognition 40 (11), 3035-3052.

Wu, Z., Leahy, R., 1993. An optimal graph theoretic approach to data clustering: Theory and its application to image segmentation. IEEE transactions on pattern analysis and machine intelligence 15 (11), 1101-1113. 
Wulder, M. A., White, J. C., Nelson, R. F., Næsset, E., Ørka, H. O., Coops, N. C., Hilker, T., Bater, C. W., Gobakken, T., 2012. Lidar sampling for large-area forest characterization: A review. Remote Sensing of Environment 121, 196-209.

Yao, W., Krull, J., Krzystek, P., Heurich, M., 2014. Sensitivity analysis of $3 \mathrm{~d}$ individual tree detection from lidar point clouds of temperate forests. Forests 5 (6), 1122-1142.

Yao, W., Krzystek, P., Heurich, M., 2012. Tree species classification and estimation of stem volume and dbh based on single tree extraction by exploiting airborne full-waveform lidar data. Remote Sensing of Environment $123,368-380$

Yao, W., Krzystek, P., Heurich, M., 2013. Enhanced detection of 3d individual trees in forested areas using airborne full-waveform lidar data by combining normalized cuts with spatial density clustering. ISPRS Annals of Photogrammetry, Remote Sensing and Spatial Information Sciences 1, 349-354.

Yao, W., Stilla, U., 2010. Mutual enhancement of weak laser pulses for point cloud enrichment based on full-waveform analysis. IEEE Transactions on Geoscience and Remote Sensing 48 (9), 3571-3579.

Yu, X., Hyyppä, J., Litkey, P., Kaartinen, H., Vastaranta, M., Holopainen, M., 2017. Single-sensor solution to tree species classification using multispectral airborne laser scanning. Remote Sensing 9 (2), 108.

Yu, X., Hyyppä, J., Vastaranta, M., Holopainen, M., Viitala, R., 2011. Predicting individual tree attributes from airborne laser point clouds based on the random forests technique. ISPRS Journal of Photogrammetry and Remote Sensing 66 (1), $28-37$.

Zhang, J., 2010. Multi-source remote sensing data fusion: status and trends. International Journal of Image and Data Fusion 1 (1), 5-24.

Zhang, K., Chen, S.-C., Whitman, D., Shyu, M.-L., Yan, J., Zhang, C., 2003. A progressive morphological filter for removing nonground measurements from airborne lidar data. IEEE transactions on geoscience and remote sensing 41 (4), 872-882.

Zhao, K., Popescu, S., Nelson, R., 2009. Lidar remote sensing of forest biomass: A scale-invariant estimation approach using airborne lasers. Remote Sensing of Environment 113 (1), 182-196.

Zhou, W., Huang, G., Troy, A., Cadenasso, M., 2009. Object-based land cover classification of shaded areas in high spatial resolution imagery of urban areas: A comparison study. Remote Sensing of Environment 113 (8), 1769-1777.

Zimble, D. A., Evans, D. L., Carlson, G. C., Parker, R. C., Grado, S. C. Gerard, P. D., 2003. Characterizing vertical forest structure using smallfootprint airborne lidar. Remote sensing of Environment 87 (2-3), 171-182. 


\section{Biography}

Nina Amiri was born on the 22th of January 1988 in Tabriz, Iran. She received her B.Sc degree in Geomatics Engineering in Tabriz University, Iran and her M.Sc. degree in Geoinformation Science and Earth Observation for Environmental Modelling and Management from Lund University, Sweden and ITC, University of Twente, the Netherlands. In 2014, she started to pursue her Ph.D. degree at the Faculty of Geo-Information Science and Earth Observation (ITC), University of Twente in collaboration with the faculty of Geo-informatics, Munich University of Applied Sciences. Her research is focused on the exploration of Lidar data in forest applications.

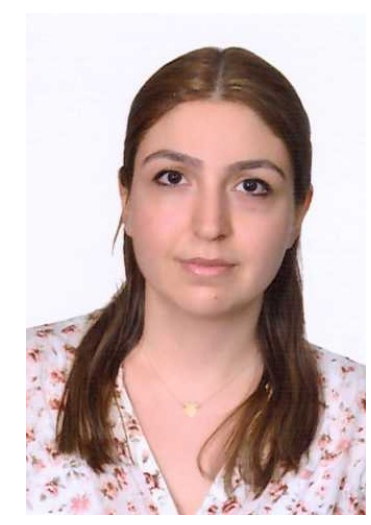

\section{Peer-reviewed papers:}

- Amiri, N., Yao, W., Heurich, M., Krzystek, P., \& Skidmore, A. K. (2016). Estimation of regeneration coverage in a temperate forest by 3D segmentation using airborne laser scanning data. International journal of applied earth observation and geoinformation, 52, 252-262.

- Amiri, N., Polewski, P., Yao, W., Krzystek, P., \& Skidmore, A. K. (2017). Detection of single tree stems in forested areas from high density ALS point clouds using 3d shape descriptors. ISPRS Annals Photogram., Remote Sens. Spatial Inform. Sci, 35-42.

- Amiri, N., Polewski, P., Heurich, M., Krzystek, P., \& Skidmore, A. K. (2018). Adaptive stopping criterion for top-down segmentation of ALS point clouds in temperate coniferous forests. ISPRS Journal of Photogrammetry and Remote Sensing, 141, 265-274.

- Amiri, N., Krzystek, P., Heurich, M., \& Skidmore, A. K. (Revised manuscript submitted). Tree species classification by fusing multis- 
pectral lidar and aerial imagery. Submitted to: ISPRS Journal of Photogrammetry and Remote Sensing

\section{Conference Proceedings:}

- Amiri, N., Yao, W., Heurich, M., \& Krzystek, P., (2015). Regeneration detection by 3D segmentation in a temperate forest using airborne full waveform Lidar data. In SilviLaser 2015, 28-30 September 2015, La Grande Motte, France.

- Amiri, N., Polewski, M. P., Yao, W., Heurich, M., Krzystek, P., \& Skidmore, A. K. (2016). Adaptive stopping criterion for normalized cut segmentation of single trees in ALS point clouds of temperate coniferous forests. Poster presentation at 3rd workshop SIG on forestry, 15-16 September 2016, Krakow, Poland.

- Amiri, N., Polewski, P., Yao, W., Heurich, M., Krzystek, P. \& Skidmore, A. (2016). Feature relevance assessment for single tree species classification using ALS point clouds and aerial imagery. Proceedings of the Young Professionals conference on remote sensing 2016, 20-21 October 2016.

- Amiri, N., Krzystek, P., Heurich, M., \& Skidmore, A. Feature Relevance Assessment Of Multispectral Airborne Lidar Data For Tree Species Classification. (2018). International Archives of Photogrammetry and Remote Sensing, (Vol. XLII-3, pp. 31-34). 